Darío Correa-Restrepo and Dieter Pfirsch

New method of deriving local energy and momentumconserving Maxwell-collisionless drift-kinetic and gyrokinetic theories: conservation laws and their structures

IPP $5 / 106$

June 2004

(Accepted for publication, J. Plasma Phys. 70, part 6, 2004 ) 


\title{
New method of deriving local energy and momentum-conserving Maxwell-collisionless drift-kinetic and gyrokinetic theories: conservation laws and their structures
}

\author{
Darío Correa-Restrepo and Dieter Pfirsch \\ Max-Planck-Institut für Plasmaphysik, EURATOM Association, D-85748 Garching, Germany \\ (dcr@ipp.mpg.de) \\ (Accepted for publication, J. Plasma Phys. 70, part 6, 2004 )
}

\begin{abstract}
This paper gives a first application of the reduced-phase-space Lagrangian for kinetic theories obtained in $[1,2]$ by means of Kruskal's averaging coordinates. The approximations made within Kruskal's formalism are of first order in the smallness parameter $\epsilon$, given by the ratio of the gyroperiod to the macroscopic time scale, which is the same as the ratio of the gyroradius to the macroscopic scale length in the driftkinetic case, or as the ratio of the amplitudes of the fluctuations to the background fields in the gyrokinetic case. The paper presents methods and results concerning local conservation laws for the density of gyrocentres and the charge, energy, momentum and angular momentum. A very important feature of our treatment is that the theory is throughout gauge invariant. The methods consist in a modified Noether formalism with gauge-invariant shift variations which in a very straightforward way lead to, in particular, the symmetric energy-momentum tensor instead of the canonical tensor. The shift variations are defined both within the reduced phase space, which does not contain the gyroangle, and also for gyroangle-dependent quantities which subsequently have to be averaged. A clear definition of the Lagrange density needed for the derivation of the local conservation laws for energy, momentum and angular momentum is given. The discovery of combinations of terms such as the polarization and the magnetization allows the conservation laws to be cast in a very clear form affording insight into their structure.
\end{abstract}




\section{Contents}

1 Introduction 3

2 Maxwell-kinetic theory $\quad 4$

2.1 The Lagrangian . . . . . . . . . . . . . . . . . 4

2.2 Some explicit expressions . . . . . . . . . . . . 6

2.3 Gauge invariance of the Lagrangian . . . . . . . . . . . 7

$\begin{array}{llr}3 & \text { The variational principle } & 8\end{array}$

$\begin{array}{lll}4 & \text { Euler-Lagrange equations } & \mathbf{1 0}\end{array}$

4.1 Variations with respect to $f_{\mathrm{p}}$ and $S \ldots \ldots \ldots$

4.1.1 Hamilton-Jacobi equation .................. 10

4.1.2 Kinetic equations . . . . . . . . . . . . 10

4.2 Variations with respect to $\Phi$ and $\mathbf{A} \ldots \ldots . . . . . . .10$

4.2.1 Charge density . . . . . . . . . . . 13

4.2 .2 Current density . . . . . . . . . . . . . 14

5 Local conservation laws $\quad \mathbf{1 6}$

5.1 Conservation of the density of gyration centres . . . . . . . 16

5.2 Charge conservation . . . . . . . . . . . 16

5.3 Local energy, momentum and angular momentum conservation . 17

5.3.1 Local energy conservation . . . . . . . . . . . 21

5.3.2 Local momentum conservation . . . . . . . . . . . 23

5.3.3 Local angular momentum conservation . . . . . . . . 24

5.3.4 Alternative form for the local momentum conservation law 26

5.3.5 Energy-momentum tensor . . . . . . . . . . . 29

6 Summary 30

$\begin{array}{lll}\text { Appendix A Symbols and useful definitions } & 31\end{array}$

$\begin{array}{lll}\text { Appendix B Some useful transformations } & 34\end{array}$

$\begin{array}{lll}\text { Appendix C Gauge-invariant shift variations } & 36\end{array}$

$\begin{array}{ll}\text { Appendix D } & \begin{array}{l}\text { Energy conservation from the } \\ \text { equations of motion }\end{array}\end{array}$

$\begin{array}{lll}\text { Appendix E Transforming some expressions } & 44\end{array}$ 


\section{Introduction}

This is the second of two closely related papers. The previous one $[1,2]$ deals with the basic theory, which is throughout gauge invariant, and in which a new method of deriving local energy and momentum-conserving Maxwellcollisionless drift-kinetic and gyrokinetic theories is described. This is in contrast to the usual treatment based on pseudo-canonical and Lie-transform theory [3-9]. Our method is closely related to the theory derived in [10] and [11], which uses, in particular, as a tool, the Hamilton-Jacobi description of the particle motion. At the beginning of the present paper an expression is given for the exact Lagrangian for the kinetic theories based on the reduced-phasespace gyroangle-independent particle Lagrangian, which agrees in the case of the drift-kinetic theory with the Lagrangian obtained by Larsson [12]. The expression for the exact Lagrangian was the concluding result of the previous paper mentioned. There, use was made of Kruskal's formalism [13] in its inverse form, as also introduced by Larsson [12], for obtaining averaging coordinates by a formal infinite-series method with a parameter of smallness $\epsilon$ given by the ratio of the gyroperiod to the macroscopic time scale, which is the same as the ratio of the gyroradius to the macroscopic scale length in the drift-kinetic case, or as the ratio of the amplitudes of the fluctuations to the background fields in the gyrokinetic case. From this Lagrangian for the kinetic theories together with the Maxwell Lagrangian, the coupled system of Maxwell's and kinetic equations can be derived by Hamilton's principle. An important point is to define the kinetic Lagrange density needed in Noether's formalism for deriving local conservation laws for energy, momentum and angular momentum. We were unable to find a clear definition of such a density for the gyrokinetic theory in the literature. We use a modified form of Noether formalism with gauge-invariant shift variations in time and space which directly yield the symmetric energy-momentum tensor instead of the nonsymmetric canonical tensor in the case of the exact Vlasov theory [14] and of the drift-kinetic theory, as follows from this paper, and in agreement with the results derived in [11]; in the case of the gyrokinetic theory the method leads to the symmetric energy-momentum tensor, albeit not directly, but nevertheless in a very straightforward way. Special consideration concerns the shift variations for the kinetic part within the reduced phase space. As opposed to the definition of the shift variations in the full phase space, a rather subtle problem is posed by their definition in the present case, which involves not only gyroangle-independent quantities defined in the reduced phase space, but also gyroangle-dependent quantities which subsequently have to be averaged. In solving this problem it is crucial that the whole theory is throughout gauge invariant. This allows one to cast the conservation laws into the mentioned transparent form. A result of our method is the discovery of a combination of terms such as the electric polarization $\mathbf{P}$ and the magnetization $\mathbf{M}$.

The paper is organized as follows: Section 2 presents the needed results from 
the previous, basic paper $[1,2]$ concerning the Maxwell-kinetic theory; a proof of the gauge invariance of the Lagrangian of this theory is added. Section 3 describes the general structure of the variational principle and the elimination of the Hamilton-Jacobi functions. Section 4 contains the derivation of the Euler-Lagrange equations consisting of the Hamilton-Jacobi equations, the kinetic equations and the inhomogeneous Maxwell equations with the expression for the charge and current densities. Section 5 contains the derivation of all conservation laws and of the energy-momentum tensor, which is symmetric in the spatial components, this being the only symmetry required for nonrelativistic theories. There are five appendices: Appendix A introduces definitions which are useful for describing variations involving the variations $\delta \Phi$ and $\delta \mathbf{A}$ of the electric and magnetic potential, respectively. Appendix B presents transformations which are used in the derivation of the variation of the kinetic Lagrange density with respect to $\Phi$ and A. Appendix C contains the definition of the gauge-invariant shift variations. For illustration, in Appendix $\mathrm{D}$ the local energy conservation law is also derived in a pedestrian way from the equations of motion of the gyrocentres. It demonstrates the elegance of Noether's formalism. Without knowledge of the final result we would hardly have been able to find the necessary steps in this direct derivation. Finally, in Appendix E, some expressions are derived which are very useful for giving the conservation laws a clear form.

\section{Maxwell-kinetic theory}

\subsection{The Lagrangian}

The following Lagrangian for general Maxwell-kinetic theories in a reduced phase space was obtained in $[1,2]$ :

$$
L_{\text {tot. }}(t)=L_{\mathrm{M}}(t)+L_{\mathrm{K}}(t),
$$

with

$$
L_{\mathrm{M}}=\int d^{3} r \frac{1}{8 \pi}\left[\mathbf{E}^{2}(\mathbf{r}, t)-\mathbf{B}^{2}(\mathbf{r}, t)\right]
$$

and

$$
\begin{aligned}
L_{\mathrm{K}}(t)= & -\sum_{\mathrm{p} . \mathrm{s} .} \int d^{3} R d U d^{4} \alpha d J f_{\mathrm{p}}\left(\mathbf{R}, U ; \alpha_{i} ; J ; t\right) \\
& \times\left[\frac{\partial S}{\partial t}\left(\mathbf{R}, U ; \alpha_{i} ; J ; t\right)+H_{\mathrm{D} S}\left(\mathbf{R}, U ; \alpha_{i} ; J ; t\right)\right] .
\end{aligned}
$$

Here, $\sum_{\mathrm{p} \text {.s. }}$ denotes summation over the particle species and $f_{\mathrm{p}}\left(\mathbf{R}, U ; \alpha_{i} ; J ; t\right)$ is the primary form of the distribution functions. The explicit expressions for 
the other quantities appearing in $L_{\mathrm{K}}(t)$ are

$$
\begin{aligned}
& H_{\mathrm{D} S}\left(\mathbf{R}, U ; \alpha_{i} ; J ; t\right)=H_{\mathrm{D}}\left(\text { with } \mathbf{P}_{\mathbf{R}} \rightarrow \frac{\partial S}{\partial \mathbf{R}}, P_{U} \rightarrow \frac{\partial S}{\partial U}\right) \\
& =e \hat{\Phi}+\mathbf{V}_{\mathbf{R}} \cdot\left(\frac{\partial S}{\partial \mathbf{R}}-\frac{e}{c} \hat{\mathbf{A}}\right)+V_{U}\left(\frac{\partial S}{\partial U}-\frac{e}{c} \hat{A}_{\mathrm{U}}\right), \\
& \mathbf{V}_{\mathbf{R}}(\mathbf{R}, U ; J ; t)=\frac{e}{m \mathcal{B}}\left[\hat{\mathbf{B}}\left(\frac{\partial \hat{\Phi}}{\partial U}+\frac{1}{c} \frac{\partial \hat{A}_{\mathrm{U}}}{\partial t}\right)+\hat{\mathbf{E}} \times\left(\frac{\partial \hat{\mathbf{A}}}{\partial U}-\frac{\partial \hat{A}_{\mathrm{U}}}{\partial \mathbf{R}}\right)\right] \\
& V_{U}(\mathbf{R}, U ; J ; t)=\frac{e}{m \mathcal{B}} \hat{\mathbf{B}} \cdot \hat{\mathbf{E}} \\
& \hat{\mathbf{A}}(\mathbf{R}, U ; J ; t)=\frac{c}{e}\left\langle\frac{\partial \mathfrak{x}}{\partial \mathbf{R}} \cdot \mathfrak{p}\right\rangle, \\
& \hat{A}_{\mathrm{U}}(\mathbf{R}, U ; J ; t)=\frac{c}{e}\left\langle\frac{\partial \mathfrak{x}}{\partial U} \cdot \mathfrak{p}\right\rangle \\
& e \hat{\Phi}(\mathbf{R}, U ; J ; t)=\langle H\rangle-\left\langle\mathfrak{p} \cdot \frac{\partial \mathfrak{x}}{\partial t}\right\rangle \\
& \mathcal{B}=\hat{\mathbf{B}} \cdot \frac{e}{m c}\left(\frac{\partial \hat{\mathbf{A}}}{\partial U}-\frac{\partial \hat{A}_{\mathrm{U}}}{\partial \mathbf{R}}\right), \\
& \hat{\mathbf{B}}=\nabla_{\mathbf{R}} \times \hat{\mathbf{A}} \text {, } \\
& \hat{\mathbf{E}}=-\frac{1}{c} \frac{\partial \hat{\mathbf{A}}}{\partial t}-\nabla_{\mathbf{R}} \hat{\Phi}, \\
& \mathbf{v}=u_{\|} \mathbf{b}+u_{\perp} \mathbf{n}_{1}+\mathbf{v}_{E}, \\
& \mathbf{p}=m \mathbf{v}+\frac{e}{c} \mathbf{A}(\mathbf{x}, t) \text {, } \\
& H=\frac{m}{2} \mathbf{v}^{2}+e \Phi(\mathbf{x}, t), \\
& \mathbf{b}=\frac{\mathbf{B}(\mathbf{x}, t)}{B(\mathbf{x}, t)}, \\
& \mathbf{n}_{1}(\mathbf{x}, \theta, t)=-\sin \theta \mathbf{e}_{1}-\cos \theta \mathbf{e}_{2}, \\
& \mathbf{e}_{1}=\frac{\mathbf{b} \cdot \nabla \mathbf{b}}{|\mathbf{b} \cdot \nabla \mathbf{b}|}, \quad \mathbf{e}_{2}=\mathbf{b} \times \mathbf{e}_{1}, \quad \mathbf{e}_{3}=\mathbf{b} .
\end{aligned}
$$

The quantities $u_{\|}, u_{\perp}, \theta$, and $\mathbf{x}$ in (13)-(18) have to be expressed in terms of the time $t$ and the averaging Kruskal variables $\mathbf{R}, U, J, \phi$, which depend on the electric and magnetic fields and their derivatives. One then has, in particular,

$$
\begin{gathered}
\mathbf{x}=\mathfrak{x}(\mathbf{R}, U, J, \phi ; t)=\mathbf{R}+\boldsymbol{\rho}(\mathbf{R}, U, J, \phi ; t), \quad \mathbf{v}=\mathfrak{v}(\mathbf{R}, U, J, \phi ; t) \\
\mathbf{p}=\mathfrak{p}(\mathbf{R}, U, J, \phi ; t)=m \mathfrak{v}+\frac{e}{c} \mathbf{A}(\mathfrak{x}, t)
\end{gathered}
$$


Explicitly, the vector $\mathfrak{v}$ is given by the expression

$$
\begin{aligned}
\mathfrak{v}= & u_{\|}(\mathbf{R}, U, J, \phi ; t) \mathbf{b}(\mathfrak{x}, t) \\
& +u_{\perp}\left[-\sin \theta(\mathbf{R}, U, J, \phi ; t) \mathbf{e}_{1}(\mathfrak{x}, t)-\cos \theta(\mathbf{R}, U, J, \phi ; t) \mathbf{e}_{2}(\mathfrak{x}, t)\right] \\
& +c \frac{\mathbf{E}(\mathfrak{x}, t) \times \mathbf{B}(\mathfrak{x}, t)}{\mathbf{B}^{2}(\mathfrak{x}, t)}
\end{aligned}
$$

with

$$
\mathbf{e}_{1}(\mathfrak{x}, t)=\frac{\mathbf{b}(\mathfrak{x}, t) \cdot \nabla \mathbf{b}(\mathfrak{x}, t)}{|\mathbf{b}(\mathfrak{x}, t) \cdot \nabla \mathbf{b}(\mathfrak{x}, t)|} \quad, \quad \mathbf{e}_{2}(\mathfrak{x}, t)=\mathbf{b}(\mathfrak{x}, t) \times \mathbf{e}_{1}(\mathfrak{x}, t)
$$

The symbol $\langle\cdots\rangle$ denotes averaging with respect to the gyroangle $\phi$ at constant $\mathbf{R}, U, J$ and $t$ :

$$
<\cdots>=\int_{0}^{1} \cdots d \phi
$$

Note that it is necessary to differentiate between $\mathfrak{v}(\mathbf{R}, U, J, \phi ; t)$ and $d \mathfrak{x} / d t=d \mathbf{R} / d t$ $+d \boldsymbol{\rho}(\mathbf{R}, U, J, \phi ; t) / d t=\mathbf{V}_{\mathbf{R}}+d \boldsymbol{\rho} / d t$. The velocities $\mathfrak{v}$ and $d \mathfrak{x} / d t$ are equal for exact single-particle theories in form of equations of motion and for exact Lagrangian theories. In the latter case $\mathfrak{v}(\mathbf{R}, U, J, \phi ; t)$ is one of the dependent quantities to be varied; the equality $\mathfrak{v}(\mathbf{R}, U, J, \phi ; t)=d \mathfrak{x} / d t$ follows from Hamilton's principle as one of the Euler-Lagrange equations. In approximate theories this Euler-Lagrange equation is modified and $\mathfrak{v}$ is no longer equal to $d \mathfrak{x} / d t$. For instance, in guiding-centre theory one might have $\mathfrak{v}=U \mathbf{b}(\mathbf{R}, t)+\mathbf{v}_{E}(\mathbf{R}, t)$ in the Lagrangian, while $\mathbf{V}_{\mathbf{R}}=\dot{\mathbf{R}}(\mathbf{R}, U, J ; t)$ contains all the drifts obtained from the Euler-Lagrange equations. The distinction between $\mathfrak{v}$ and $d \mathfrak{x} / d t$ is important in the following treatment.

\subsection{Some explicit expressions}

The results needed are presented in $[1,2]$ :

\section{Zeroth order:}

$$
\mathfrak{x}=\mathbf{R}, u_{\|}=U, u_{\perp}=\sqrt{\frac{\Omega(\mathbf{R}, t) J}{\pi m}}, \vartheta=\phi
$$

with

$$
\Omega(\mathbf{R}, t)=\frac{e B}{m c}=\mathcal{O}\left(\frac{1}{\epsilon}\right), J=\frac{2 \pi m c}{e} \times \text { lowest-order magnetic moment. }
$$

Within the Kruskal formalism the lowest-order magnetic moment is of first order in $\epsilon$ (see below). 


\section{First order:}

The first-order corrections are needed only for $\mathfrak{x}$. Up to the first order one has

$$
\begin{aligned}
\mathfrak{x} & =\mathbf{R}+\frac{1}{\Omega(\mathbf{R})} u_{\perp} \mathbf{n}_{2}(\mathbf{R}, \phi, t) \\
& =\mathbf{R}+\boldsymbol{\rho} \\
\mathbf{n}_{2}(\mathbf{R}, \phi, t) & =\cos 2 \pi \phi \mathbf{e}_{1}(\mathbf{R}, t)-\sin 2 \pi \phi \mathbf{e}_{2}(\mathbf{R}, t),
\end{aligned}
$$

from which it follows, in particular, that

$$
J=\frac{2 \pi}{\Omega(\mathbf{R}, t)} \frac{m u_{\perp}^{2}}{2} .
$$

All the other quantities are again the zeroth-order quantities.

\subsection{Gauge invariance of the Lagrangian}

It is only necessary to prove that

$$
\frac{\partial S}{\partial t}\left(\mathbf{R}, U ; \alpha_{i} ; J ; t\right)+H_{\mathrm{D} S}\left(\mathbf{R}, U ; \alpha_{i} ; J ; t\right)
$$

is gauge invariant. We show that this expression is left unchanged by the gauge transformation

$$
\begin{aligned}
& \mathbf{A}(\mathbf{x}, t) \rightarrow \mathbf{A}(\mathbf{x}, t)+\nabla \Psi(\mathbf{x}, t) \\
& \Phi(\mathbf{x}, t) \rightarrow \Phi(\mathbf{x}, t)-\frac{1}{c} \frac{\partial \Psi(\mathbf{x}, t)}{\partial t} \\
& S\left(\mathbf{R}, U, J ; \alpha_{i} ; t\right) \rightarrow S\left(\mathbf{R}, U, J ; \alpha_{i} ; t\right)+\frac{e}{c}\langle\Psi(\mathbf{x}=\mathfrak{x}(\mathbf{R}, U, J, \phi ; t), t)\rangle
\end{aligned}
$$

With this transformation, one obtains

$$
\begin{aligned}
\frac{\partial S}{\partial t} & +e \hat{\Phi}=\frac{\partial S}{\partial t}+\left\langle\frac{m}{2} \mathfrak{v}^{2}+e \Phi(\mathbf{x}=\mathfrak{x}, t)\right\rangle-\left\langle\frac{\partial \mathfrak{x}}{\partial t} \cdot \mathfrak{p}\right\rangle \\
& \rightarrow \frac{\partial S}{\partial t}+\left.\frac{e}{c} \frac{\partial}{\partial t}\right|_{\mathbf{R}, U, J}\langle\Psi(\mathbf{x}=\mathfrak{x}(\mathbf{R}, U, J, \phi ; t), t)\rangle \\
& +e \hat{\Phi}-\frac{e}{c}\left\langle\left.\frac{\partial}{\partial t}\right|_{\mathbf{x}} \Psi(\mathbf{x}, t)+\left.\frac{\partial \mathfrak{x}}{\partial t}\right|_{\mathbf{R}, U, J, \phi} \frac{\partial \Psi(\mathbf{x}, t)}{\partial \mathbf{x}}\right\rangle=\frac{\partial S}{\partial t}+e \hat{\Phi}
\end{aligned}
$$

since the time derivatives of $\Psi(\mathbf{x}, t)$ cancel each other. Here, use was made of the fact that the potentials do not enter either $\mathfrak{x}$ or $\mathfrak{v}$; only the fields appear in these quantities. 
Furthermore, one has

$$
\begin{aligned}
\frac{\partial S}{\partial \mathbf{R}} & -\frac{e}{c} \hat{\mathbf{A}}=\frac{\partial S}{\partial \mathbf{R}}-\left\langle\frac{\partial \mathfrak{x}}{\partial \mathbf{R}} \cdot \mathfrak{p}\right\rangle \\
& \rightarrow \frac{\partial S}{\partial \mathbf{R}}+\left.\frac{e}{c} \frac{\partial}{\partial \mathbf{R}}\right|_{U, J, t}\langle\Psi(\mathbf{x}=\mathfrak{x}(\mathbf{R}, U, J, \phi ; t), t)\rangle \\
& -\frac{e}{c} \hat{\mathbf{A}}-\frac{e}{c}\left\langle\left.\left.\frac{\partial \mathfrak{x}}{\partial \mathbf{R}}\right|_{U, J, \phi, t} \cdot \frac{\partial}{\partial \mathbf{x}}\right|_{t} \Psi(\mathbf{x}, t)\right\rangle=\frac{\partial S}{\partial \mathbf{R}}-\frac{e}{c} \hat{\mathbf{A}}
\end{aligned}
$$

The gauge invariance of $\partial S / \partial U-(e / c) \hat{A}_{\mathrm{U}}$ can be shown in a similar way. Since the potentials do not appear in the velocities $\mathbf{V}_{\mathbf{R}}$ and $V_{U}$, it is thus shown that the Lagrangian is gauge invariant.

\section{The variational principle}

The variational principle for the total Maxwell-kinetic Lagrangian is

$$
\begin{gathered}
\delta \mathcal{A}_{\text {tot. }}=\delta \mathcal{A}_{\mathrm{M}}+\delta \mathcal{A}_{\mathrm{K}}=0 \\
\mathcal{A}_{\mathrm{M}}=\int_{t_{1}}^{t_{2}} L_{\mathrm{M}} d t \quad, \quad \mathcal{A}_{\mathrm{K}}=\int_{t_{1}}^{t_{2}} L_{\mathrm{K}} d t
\end{gathered}
$$

with $\delta f_{\mathrm{p}}=\delta S=\delta \Phi=\delta \mathbf{A}=0$ on the boundaries of the whole integration space, including time. Here, the symbol $\delta$ is used to denote variations, in contrast to $\delta$, which is reserved for the $\delta$-functions. When the Euler-Lagrange equations obtained from the variational principle are taken into account in order to obtain "macroscopic" quantities, such as charge and current densities, integrals containing the primary distribution functions $f_{\mathrm{p}}\left(\mathbf{R}, U ; \alpha_{i} ; J ; t\right)$ appear. In these integrals, the $\alpha_{i}$ are eliminated by re-introducing the original canonical momenta $\mathbf{P}_{\mathbf{R}}$ and $P_{U}$, which have to satisfy the Dirac constraints $\mathbf{P}_{\mathbf{R}}-\frac{e}{c} \hat{\mathbf{A}}=0$ and $P_{U}-\frac{e}{c} \hat{A}_{\mathrm{U}}=0$. As explained in [1,2], Sec. 2.4, a consequence of this is that the following substitutions must be made in the expressions concerned:

$$
\begin{aligned}
& \frac{\partial S}{\partial \mathbf{R}} \rightarrow \mathbf{P}_{\mathbf{R}} \\
& \frac{\partial S}{\partial U} \rightarrow P_{U} \\
& f_{\mathrm{p}} d^{4} \alpha \rightarrow \\
& \mathcal{B}(\mathbf{R}, U ; J ; t) \delta\left(\mathbf{P}_{\mathbf{R}}-\frac{e}{c} \hat{\mathbf{A}}\right) \delta\left(P_{U}-\frac{e}{c} \hat{A}_{\mathrm{U}}\right) f(\mathbf{R}, U ; J ; t) d^{3} P_{\mathbf{R}} d P_{U} .
\end{aligned}
$$

Here, $f(\mathbf{R}, U ; J ; t)$ is the distribution function, which, as follows from the variational principle, is the solution of the kinetic equation

$$
\frac{\partial f}{\partial t}+\mathbf{V}_{\mathbf{R}} \cdot \frac{\partial f}{\partial \mathbf{R}}+V_{U} \frac{\partial f}{\partial U}=0
$$


The function $\mathcal{B}(\mathbf{R}, U ; J ; t)$, which is the Jacobian for the coordinate transformation from $(\mathbf{x}, \mathbf{v} ; t)$ to the averaging coordinates $(\mathbf{R}, U ; J, \phi ; t)$, was shown in $[1,2]$ to satisfy a continuity equation in the space $(\mathbf{R}, U ; J ; t)$. Therefore, the phase-space volume element $d \tau$ defined as

$$
d \tau:=\left|\mathcal{B}\left(R_{1}, \ldots, R_{3}, U ; J ; t\right)\right| d R_{1} d R_{2} d R_{3} d U d J
$$

is Liouvillian.

The substitution procedure in expressions (35) is similar to that employed in deriving the kinetic equation for $f$, (36), from the continuity equation for $f_{\mathrm{p}}\left(\mathbf{R}, U ; \alpha_{i} ; J ; t\right)$, this being obtained further below as one of the EulerLagrange equations derived from (33).

As an illustration of the substitution procedure we now consider variations with respect to the potentials $\Phi$ and $\mathbf{A}$ in the kinetic part of the action integral. Let $\delta_{\text {pot. }} \mathcal{A}_{\mathrm{K}}$ be the variation of the integral $\mathcal{A}_{\mathrm{K}}$ owing to a variation of the potentials $\Phi$ and $\mathbf{A}$ :

$$
\begin{aligned}
\delta_{\text {pot. }} \mathcal{A}_{\mathrm{K}}= & -\sum_{\text {p. s. }} \int d t d^{3} R d U d^{4} \alpha d J f_{\mathrm{p}}\left(\mathbf{R}, U ; \alpha_{i} ; J ; t\right) \delta_{\text {pot. }} H_{\mathrm{D} S}\left(\mathbf{R}, U ; \alpha_{i} ; J ; t\right) \\
= & -\sum_{\text {p. s. }} \int d t d^{3} R d U d^{4} \alpha d J f_{\mathrm{p}}\left[\delta_{\text {pot. }} e \hat{\Phi}-\mathbf{V}_{\mathbf{R}} \cdot \frac{e}{c} \delta_{\text {pot. }} \hat{\mathbf{A}}-V_{U} \frac{e}{c} \delta_{\text {pot. }} \hat{A}_{\mathrm{U}}\right. \\
& \left.+\left(\delta_{\text {pot. }} \mathbf{V}_{\mathbf{R}}\right) \cdot\left(\frac{\partial S}{\partial \mathbf{R}}-\frac{e}{c} \hat{\mathbf{A}}\right)+\left(\delta_{\text {pot. }} V_{U}\right)\left(\frac{\partial S}{\partial U}-\frac{e}{c} \hat{A}_{\mathrm{U}}\right)\right] .
\end{aligned}
$$

Transformation of the integrals, eliminating $f_{\mathrm{p}}$ and $\alpha_{i}$ and introducing $f, \mathbf{P}_{\mathbf{R}}$ and $P_{U}$ instead, yields

$$
\begin{aligned}
\delta_{\text {pot. }} \mathcal{A}_{\mathrm{K}}= & -\sum_{\text {p.s. }} \int d t d^{3} R d U d^{3} P_{\mathbf{R}} d P_{U} d J \mathcal{B}(\mathbf{R}, U ; J ; t) \\
& \times f(\mathbf{R}, U ; J ; t) \delta\left(\mathbf{P}_{\mathbf{R}}-\frac{e}{c} \hat{\mathbf{A}}\right) \delta\left(P_{U}-\frac{e}{c} \hat{A}_{\mathrm{U}}\right) \delta_{\text {pot. }} H_{\mathrm{D}}\left(\mathbf{R}, U ; \mathbf{P}_{\mathbf{R}}, P_{U} ; J ; t\right) \\
= & -\sum_{\text {p.s. }} \int d t d^{3} R d U d J \mathcal{B}(\mathbf{R}, U ; J ; t) f(\mathbf{R}, U ; J ; t)\left(\delta_{\text {pot. }} H_{\mathrm{D}}\right)_{\text {constr. }},
\end{aligned}
$$

where the subscript constr. means that the constraints $\mathbf{P}_{\mathbf{R}}=\frac{e}{c} \hat{\mathbf{A}}$ and $P_{U}=$ $\frac{e}{c} \hat{A}_{\mathrm{U}}$ have been applied. Equation (39) is the same as would have been obtained by setting

$$
\delta_{\text {pot. }} \mathcal{A}_{\mathrm{K}}=-\sum_{\text {p. s. }} \int d t d^{3} R d U d^{4} \alpha d J f_{\mathrm{p}}\left[\delta_{\text {pot. }} e \hat{\Phi}-\mathbf{V}_{\mathbf{R}} \cdot \frac{e}{c} \delta_{\text {pot. }} \hat{\mathbf{A}}-V_{U} \frac{e}{c} \delta_{\text {pot. }} \hat{A}_{\mathrm{U}}\right]
$$

from the outset in the expression for $\delta_{\text {pot. }} \mathcal{A}_{\mathrm{K}}$. It is clear that it is not necessary to vary the $\Phi$ and $\mathbf{A}$-dependent quantities $\mathbf{V}_{\mathbf{R}}$ and $V_{U}$ in $L_{\mathrm{K}}$, since their variations are multiplied by $f_{\mathrm{p}}$ and the constraints. Owing to the properties of $f_{\mathrm{p}}$, as described by the substitutions (35), this means multiplication by zero. 


\section{Euler-Lagrange equations}

\subsection{Variations with respect to $f_{\mathrm{p}}$ and $S$}

\subsubsection{Hamilton-Jacobi equation}

The variation of the action integral with respect to $f_{\mathrm{p}}\left(\mathbf{R}, U ; \alpha_{i} ; J ; t\right)$ is

$$
\begin{aligned}
\delta_{\delta f_{\mathrm{p}}} \mathcal{A}_{\mathrm{tot.}}= & -\sum_{\mathrm{p} . \mathrm{s.}} \int d t d^{3} R d U d^{4} \alpha d J \delta f_{\mathrm{p}}\left(\mathbf{R}, U ; \alpha_{i} ; J ; t\right) \\
& \times\left[\frac{\partial S}{\partial t}\left(\mathbf{R}, U ; \alpha_{i} ; J ; t\right)+H_{\mathrm{D} S}\left(\mathbf{R}, U ; \alpha_{i} ; J ; t\right)\right] .
\end{aligned}
$$

The vanishing of this expression yields the Hamilton-Jacobi equation

$$
\frac{\partial S}{\partial t}\left(\mathbf{R}, U ; \alpha_{i} ; J ; t\right)+H_{\mathrm{D} S}\left(\mathbf{R}, U ; \alpha_{i} ; J ; t\right)=0 .
$$

\subsubsection{Kinetic equations}

Variation of the action integral with respect to $S\left(\mathbf{R}, U ; \alpha_{i} ; J ; t\right)$ yields

$$
\begin{aligned}
\delta_{\delta S} \mathcal{A}_{\text {tot. }}= & -\sum_{\text {p.s. }} \int d t d^{3} R d U d^{4} \alpha d J f_{\mathrm{p}}\left(\mathbf{R}, U ; \alpha_{i} ; J ; t\right) \\
& \times\left[\frac{\partial \delta S}{\partial t}+\mathbf{V}_{\mathbf{R}} \cdot \frac{\partial \delta S}{\partial \mathbf{R}}+V_{U} \frac{\partial \delta S}{\partial V_{U}}\right]
\end{aligned}
$$

After some partial integrations, with $\delta S=0$ on the boundaries of the integration domain taken into account, Hamilton's principle yields the following continuity equation for the primary form $f_{\mathrm{p}}$ of the distribution functions:

$$
\frac{\partial f_{\mathrm{p}}}{\partial t}\left(\mathbf{R}, U ; \alpha_{i} ; J ; t\right)+\frac{\partial}{\partial \mathbf{R}} \cdot\left(\mathbf{V}_{\mathbf{R}} f_{\mathrm{p}}\right)+\frac{\partial}{\partial U}\left(V_{U} f_{\mathrm{p}}\right)=0
$$

By applying the standard method previously discussed in $[1,2]$, Sec. 2.4, which leads from the primary form $f_{\mathrm{p}}\left(\mathbf{R}, U ; \alpha_{i} ; J ; t\right)$ of the distribution functions to the actual distribution functions $f(\mathbf{R}, U ; J ; t)$, one obtains from (44) the kinetic equation for each particle species:

$$
\frac{\partial f}{\partial t}(\mathbf{R}, U ; J ; t)+\mathbf{V}_{\mathbf{R}} \cdot \frac{\partial f}{\partial \mathbf{R}}+V_{U} \frac{\partial f}{\partial U}=0 .
$$

\subsection{Variations with respect to $\Phi$ and $\mathrm{A}$}

The variations with respect to $\Phi$ and $\mathbf{A}$ are more difficult to do than those with respect to $f_{\mathrm{p}}$ and $S$, the reason for this being that the potentials and fields appear both as functions of $(\mathbf{R}, t)$ and/or as functions of $(\mathfrak{x}(\mathbf{R}, U, J, \phi ; t), t), \mathfrak{x}$ being itself a function of the fields. In both cases the variations have to be 
performed at the same point and time $(\mathbf{r}, t)$ as appear in the Maxwell part of the Lagrangian. For describing these variations, it is convenient to introduce some additional symbols and definitions; this is done mainly in Appendix A. As already explained in the derivation of (39) and (40), the quantity of interest is $\left(\delta_{\text {pot. }} H_{\mathrm{D}}\right)_{\text {constr. }}$, which is obtained by varying $(4)$, re-introducing $\mathbf{P}_{\mathbf{R}}$ and $P_{U}$ according to (35) and applying the Dirac constraints $\mathbf{P}_{\mathbf{R}}=\frac{e}{c} \hat{\mathbf{A}}, P_{U}=\frac{e}{c} \hat{A}_{\mathrm{U}}$. As mentioned before, it is not necessary to vary the $\Phi$ and $\mathbf{A}$-dependent quantities $\mathbf{V}_{\mathbf{R}}$ and $V_{U}$, since the variations of these quantities are multiplied by zero after applying the constraints. This procedure yields

$$
\left(\delta_{\text {pot. }} H_{\mathrm{D}}\right)_{\text {constr. }}=\delta_{\text {pot. }}\left\langle e \Phi+\frac{1}{2} m \mathfrak{v}^{2}-\frac{\partial \mathfrak{x}}{\partial t} \cdot \mathfrak{p}\right\rangle-\mathbf{V}_{\mathbf{R}} \cdot \frac{e}{c} \delta_{\text {pot. }} \hat{\mathbf{A}}-V_{U} \frac{e}{c} \delta_{\text {pot. }} \hat{A}_{\mathrm{U}}
$$

With the definitions of $\hat{\mathbf{A}}$ and $\hat{A}_{\mathrm{U}},(7)$ and (8), this can be written as

$$
\begin{aligned}
\left(\delta_{\text {pot. }} H_{\mathrm{D}}\right)_{\text {constr. }}= & \delta_{\text {pot. }}\left\langle e \Phi+\frac{1}{2} m \mathfrak{v}^{2}\right\rangle \\
& -\left\langle\left[\frac{\partial \delta_{\text {pot. }} \mathfrak{x}}{\partial t}+\mathbf{V}_{\mathbf{R}} \cdot \frac{\partial \delta_{\text {pot. }} \mathfrak{x}}{\partial \mathbf{R}}+V_{U} \frac{\partial \delta_{\text {pot. }} \mathfrak{x}}{\partial U}\right] \cdot \mathfrak{p}\right\rangle \\
& -\left\langle\left[\frac{\partial \mathfrak{x}}{\partial t}+\mathbf{V}_{\mathbf{R}} \cdot \frac{\partial \mathfrak{x}}{\partial \mathbf{R}}+V_{U} \frac{\partial \mathfrak{x}}{\partial U}\right] \cdot \delta_{\text {pot. }} \mathfrak{p}\right\rangle \\
= & \delta_{\text {pot. }}\left\langle e \Phi+\frac{1}{2} m \mathfrak{v}^{2}\right\rangle-\left\langle\left[\frac{\mathfrak{D} \delta_{\text {pot }} \mathfrak{x}}{\mathfrak{D} t}\right] \cdot \mathfrak{p}\right\rangle-\left\langle\frac{\mathfrak{D} \mathfrak{x}}{\mathfrak{D} t} \cdot \delta_{\text {pot. }} \mathfrak{p}\right\rangle(
\end{aligned}
$$

with

$$
\frac{\mathfrak{D}}{\mathfrak{D} t} \equiv \frac{d}{d t}-\dot{\phi} \frac{\partial}{\partial \phi}
$$

being the time derivative without the fast gyroangle dependence, as defined in (A8).

Some quantities in (47) depend directly on $\mathbf{R}$, while other quantities depend on $\mathfrak{x}(\mathbf{R}, U, J, \phi ; t)$, which depends on the magnetic field $\mathbf{B}(\mathbf{R}, t)$ and its derivatives through $\rho$ :

$$
\boldsymbol{\rho} \equiv \mathfrak{x}(\mathbf{R}, U, J, \phi ; t)-\mathbf{R} .
$$

Thus, when the potentials and the fields depend on $\mathfrak{x}$, they have to be varied in two respects: the functions and the arguments of the functions. We then write, for example,

$$
\begin{aligned}
\delta_{\text {pot. }} \Phi(\mathfrak{x}, t) & =\delta \Phi(\mathfrak{x}, t)+\delta_{\text {pot. }} \mathfrak{x} \cdot \frac{\partial \Phi}{\partial \mathfrak{x}} \\
& =\delta \Phi(\mathfrak{x}, t)+\delta_{\text {pot. }} \boldsymbol{\rho} \cdot \frac{\partial \Phi}{\partial \mathfrak{x}}
\end{aligned}
$$

Thus, $\delta \Phi(\mathfrak{x}, t)$ and $\delta \mathbf{A}(\mathfrak{x}, t)$, without any subscript, always means here the variation of the functions alone, without the contribution from the variation of 
the argument. For functions which only depend on the values of the potentials and fields at $\mathbf{R}$, e.g. $\mathbf{B}(\mathbf{R}, t), \delta_{\text {pot. }}$ and $\delta$ are the same. For all purposes of interest here, as will be specified later, $\mathfrak{x}$ and $\boldsymbol{\rho}$ are also such functions. Explicitly, $\delta_{\text {pot. }} \mathfrak{x}$ and $\delta_{\text {pot. }} \boldsymbol{\rho}=\delta \boldsymbol{\rho}$ are equal and are given by

$$
\begin{aligned}
\delta_{\text {pot. }} \mathfrak{x}=\delta \boldsymbol{\rho} & =\delta \mathbf{B}(\mathbf{R}, t) \cdot \frac{\partial \boldsymbol{\rho}}{\partial \mathbf{B}(\mathbf{R}, t)}+\delta \mathbf{B}_{, R_{i}} \cdot \frac{\partial \boldsymbol{\rho}}{\partial \mathbf{B}_{, R_{i}}(\mathbf{R}, t)} \\
& =\left(\boldsymbol{\nabla}_{\mathbf{R}} \times \delta \mathbf{A}\right) \cdot \frac{\partial \boldsymbol{\rho}}{\partial \mathbf{B}}+\left(\boldsymbol{\nabla}_{\mathbf{R}} \times \frac{\partial \delta \mathbf{A}}{\partial R_{i}}\right) \cdot \frac{\partial \boldsymbol{\rho}}{\partial \mathbf{B}_{, R_{i}}}
\end{aligned}
$$

with $\mathbf{B}_{R_{i}}(\mathbf{R}, t)=\partial \mathbf{B}(\mathbf{R}, t) / \partial R_{i}$. Multiplication of the third term in (47) by $f_{\mathrm{p}}$ and some transformations yields

$$
\begin{aligned}
-f_{\mathrm{p}}\left[\frac{\mathfrak{D} \delta_{\text {pot. }} \mathfrak{x}}{\mathfrak{D} t}\right] \cdot \mathfrak{p}= & -f_{\mathrm{p}}\left[\frac{\mathfrak{D} \delta \boldsymbol{\rho}}{\mathfrak{D} t}\right] \cdot \mathfrak{p} \\
= & -\frac{\partial}{\partial t}\left[f_{\mathrm{p}}(\delta \boldsymbol{\rho} \cdot \mathfrak{p})\right]-\frac{\partial}{\partial \mathbf{R}}\left[\mathbf{V}_{\mathbf{R}} f_{\mathrm{p}}(\delta \boldsymbol{\rho} \cdot \mathfrak{p})\right]-\frac{\partial}{\partial U}\left[V_{U} f_{\mathrm{p}}(\delta \boldsymbol{\rho} \cdot \mathfrak{p})\right] \\
& +f_{\mathrm{p}} \delta \boldsymbol{\rho} \cdot\left[m \frac{\mathfrak{D} \mathfrak{v}}{\mathfrak{D} t}+\frac{e}{c} \frac{\mathfrak{D} \mathfrak{x}}{\mathfrak{D} t} \cdot \frac{\partial \mathbf{A}(\mathfrak{x}, t)}{\partial \mathfrak{x}}+\frac{e}{c} \frac{\partial \mathbf{A}(\mathfrak{x}, t)}{\partial t}\right]
\end{aligned}
$$

where use has been made of the Euler-Lagrange equation for $f_{\mathrm{p}}$, (44). The fourth term in (47) can be transformed in a similar way:

$$
\begin{aligned}
-f_{\mathrm{p}} \frac{\mathfrak{D} \mathfrak{x}}{\mathfrak{D} t} \cdot \delta_{\text {pot. }} \mathfrak{p}= & -f_{\mathrm{p}} \frac{\mathfrak{D} \mathfrak{x}}{\mathfrak{D} t} \cdot\left[\delta(m \mathfrak{v})+\delta \boldsymbol{\rho} \cdot \frac{\partial_{(\mathfrak{x}, t)}(m \mathfrak{v})}{\partial \mathfrak{x}}\right. \\
& \left.+\frac{e}{c} \delta \mathbf{A}(\mathfrak{x}, t)+\frac{e}{c} \delta \boldsymbol{\rho} \cdot \frac{\partial \mathbf{A}}{\partial \mathfrak{x}}\right] .
\end{aligned}
$$

The operator $\partial_{(x, t)}$ used in this expression is defined in Appendix A.

From (46)-(53) one obtains after some steps

$$
\begin{aligned}
& f_{\mathrm{p}}\left(\delta_{\text {pot. }} H_{\mathrm{D}}\right)_{\text {constr. }}= f_{\mathrm{p}}\left\langle e \delta \Phi(\mathfrak{x}, t)-\frac{e}{c} \frac{\mathfrak{D} \mathfrak{x}}{\mathfrak{D} t} \cdot \delta \mathbf{A}(\mathfrak{x}, t)\right. \\
&\left.+\delta\left(\frac{m}{2} \mathfrak{v}^{2}\right)-\delta(m \mathfrak{v}) \cdot \frac{\mathfrak{D} \mathfrak{x}}{\mathfrak{D} t}+\delta \boldsymbol{\rho} \cdot \mathbf{G}\right\rangle \\
&-\frac{\partial}{\partial t}\left[f_{\mathrm{p}}\langle\delta \boldsymbol{\rho} \cdot \mathfrak{p}\rangle\right]-\frac{\partial}{\partial \mathbf{R}} \cdot\left[\mathbf{V}_{\mathbf{R}} f_{\mathrm{p}}\langle\delta \boldsymbol{\rho} \cdot \mathfrak{p}\rangle\right]-\frac{\partial}{\partial U}\left[V_{U} f_{\mathrm{p}}\langle\delta \boldsymbol{\rho} \cdot \mathfrak{p}\rangle\right]
\end{aligned}
$$

with $\mathbf{G}$ given by (A14). The last three terms in (54) will not contribute to the Euler-Lagrange equations, since they vanish after integration in the action integral. However, the terms $\sim \partial / \partial t$ and $\sim \partial / \partial \mathbf{R}$ do contribute to the local conservation laws, while the last term vanishes locally after $U$-integration. 


\subsubsection{Charge density}

For calculation of the charge density $f_{\mathrm{p}}\left(\delta_{\delta \Phi} H_{\mathrm{D}}\right)_{\text {constr. }}$ is required. In this expression the electric potential appears undifferentiated only in the term $\delta \Phi(\mathfrak{x}, t)$. Otherwise, there are only derivatives of $\Phi$. From (51) and (54) one obtains after some steps

$$
\begin{aligned}
f_{\mathrm{p}}\left(\delta_{\delta \Phi} H_{\mathrm{D}}\right)_{\text {constr. }}= & f_{\mathrm{p}}\left[e\langle\delta \Phi(\mathfrak{x}, t)\rangle+\frac{\partial \delta \Phi(\mathbf{R}, t)}{\partial \mathbf{R}} \cdot \frac{\widehat{\partial}_{(\mathbf{R}, U, J ; t)}\left\langle\mathcal{T}_{\mathfrak{v}}\right\rangle}{\partial \boldsymbol{\nabla}_{\mathbf{R}} \Phi(\mathbf{R}, t)}\right. \\
& \left.+\left\langle\frac{\partial \delta \Phi(\mathfrak{x}, t)}{\partial \mathfrak{x}} \cdot \frac{\widehat{\partial}_{(\mathfrak{x}, t)} \mathcal{T}_{\mathfrak{v}}}{\partial \boldsymbol{\nabla}_{\mathfrak{x}} \Phi(\mathfrak{x}, t)}\right\rangle\right]
\end{aligned}
$$

The quantity $\mathcal{T}_{\mathfrak{v}}$ is defined in (A10). The definition of the operator $\widehat{\partial}$, which acts only on the first term of a product, is given in (A12)-(A16). The variations $\delta \Phi$ have to be localized at the same point and time $(\mathbf{r}, t)$ which appear in the Maxwell part of the Lagrangian. A convenient way of doing this is to express the variations in the following way:

$$
\delta \Phi(\mathfrak{x}, t)=\int d^{3} r \delta(\mathbf{r}-\mathfrak{x}) \delta \Phi(\mathbf{r}, t), \quad \delta \Phi(\mathbf{R}, t)=\int d^{3} r \delta(\mathbf{r}-\mathbf{R}) \delta \Phi(\mathbf{r}, t) .
$$

Then, with $\partial / \partial \boldsymbol{\nabla} \Phi \sim-\partial / \partial \mathbf{E}, \partial \delta(\mathbf{r}-\mathbf{R}) / \partial \mathbf{R}=-\partial \delta(\mathbf{r}-\mathbf{R}) / \partial \mathbf{r}, \partial \delta(\mathbf{r}-\mathfrak{x}) / \partial \mathfrak{x}$ $=-\partial \delta(\mathbf{r}-\mathfrak{x}) / \partial \mathbf{r}$ and the substitution of Sec. 3, and taking into account the vanishing of some terms at the $\mathbf{r}$-boundaries, one obtains

$$
\begin{aligned}
& \int d^{4} \alpha f_{\mathrm{p}}\left(\delta_{\delta \Phi} H_{\mathrm{D} S}\right)=\mathcal{B} f\left(\delta_{\delta \Phi} H_{\mathrm{D}}\right)_{\text {constr. }} \\
&=\int d^{3} r \delta \Phi(\mathbf{r}, t)[e \mathcal{B} f\langle\delta(\mathbf{r}-\mathfrak{x})\rangle \\
&+\frac{\partial}{\partial \mathbf{r}} \cdot\left(\delta(\mathbf{r}-\mathbf{R}) \mathcal{B} f \frac{\widehat{\partial}_{(\mathbf{R}, U, J ; t)}\left\langle\mathcal{T}_{\mathfrak{v}}\right\rangle}{\partial \mathbf{E}(\mathbf{R}, t)}\right)+\frac{\partial}{\partial \mathbf{r}} \cdot\left\langle\delta(\mathbf{r}-\mathfrak{x}) \mathcal{B} f \frac{\widehat{\partial}_{(\mathfrak{x}, t)} \mathcal{T}_{\mathfrak{v}}}{\partial \mathbf{E}(\mathfrak{x}, t)}\right\rangle(57)
\end{aligned}
$$

The variational principle

$$
\begin{aligned}
0=\delta_{\delta \Phi} \mathcal{A}_{\text {tot. }}= & \delta_{\delta \Phi} \mathcal{A}_{\mathrm{M}}+\delta_{\delta \Phi} \mathcal{A}_{\mathrm{K}} \\
= & \int d t d^{3} r\left[\delta \Phi(\mathbf{r}, t) \frac{1}{4 \pi} \boldsymbol{\nabla} \cdot \mathbf{E}(\mathbf{r}, t)\right] \\
& -\sum_{\text {p.s. }} \int d t d^{3} R d U d J \mathcal{B} f\left(\delta_{\delta \Phi} H_{\mathrm{D}}\right)_{\text {constr. }}
\end{aligned}
$$

yields the charge density as a sum of particle-like and polarization charge densities:

$$
\rho(\mathbf{r}, t)=\frac{1}{4 \pi} \nabla \cdot \mathbf{E}(\mathbf{r}, t)=\rho_{\text {particle-like }}(\mathbf{r}, t)+\rho_{\text {polarization }}(\mathbf{r}, t),
$$

with

$$
\rho_{\text {particle-like }}=\sum_{\text {p.s. }} e \int d^{3} R d U d J \mathcal{B} f\langle\delta(\mathbf{r}-\mathfrak{x})\rangle
$$


and

$$
\rho_{\text {polarization }}=-\nabla_{\mathbf{r}} \cdot \mathbf{P},
$$

where the polarization $\mathbf{P}$ is given by the expression

$$
\begin{aligned}
\mathbf{P} \equiv & -\sum_{\text {p.s. }} \int d^{3} R d U d J \mathcal{B} f\left[\delta(\mathbf{r}-\mathbf{R}) \frac{\widehat{\partial}_{(\mathbf{R}, U, J ; t)}\left\langle\mathcal{T}_{\mathfrak{v}}\right\rangle}{\partial \mathbf{E}(\mathbf{R}, t)}+\left\langle\delta(\mathbf{r}-\mathfrak{x}) \frac{\widehat{\partial}_{(\mathfrak{x}, t)} \mathcal{T}_{\mathfrak{v}}}{\partial \mathbf{E}(\mathfrak{x}, t)}\right\rangle\right] \\
= & -\sum_{\text {p.s. }}\left[\int d U d J \mathcal{B} f \frac{\widehat{\partial}_{(\mathbf{R}, U, J ; t)}\left\langle\mathcal{T}_{\mathfrak{v}}\right\rangle}{\partial \mathbf{E}(\mathbf{R}, t)}\right]_{\mathbf{R}=\mathbf{r}} \\
& -\sum_{\text {p.s. }} \int d^{3} R d U d J \mathcal{B} f\left\langle\delta(\mathbf{r}-\mathfrak{x}) \frac{\widehat{\partial}_{(\mathfrak{x}, t)} \mathcal{T}_{\mathfrak{v}}}{\partial \mathbf{E}(\mathfrak{x}, t)}\right\rangle
\end{aligned}
$$

\subsubsection{Current density}

Equation (54) yields preliminary results for obtaining the current density:

$$
\begin{aligned}
\int d^{4} \alpha & f_{\mathrm{p}}\left(\delta_{\delta \mathbf{A}} H_{\mathrm{D} S}\right)=\mathcal{B} f\left(\delta_{\delta \mathbf{A}} H_{\mathrm{D}}\right)_{\text {constr. }} \\
= & \mathcal{B} f\left\{-\frac{e}{c} \mathbf{V}_{\mathbf{R}} \cdot\langle\delta \mathbf{A}(\mathfrak{x}, t)\rangle-\frac{e}{c}\left\langle\frac{\mathfrak{D} \boldsymbol{\rho}}{\mathfrak{D} t} \cdot \delta \mathbf{A}(\mathfrak{x}, t)\right\rangle\right. \\
& -\left.\frac{1}{c} \frac{\partial \delta \mathbf{A}(\mathbf{R}, t)}{\partial t}\right|_{\mathbf{R}} \cdot \frac{\widehat{\partial}_{(\mathbf{R}, U, J ; t)}\left\langle\mathcal{T}_{\mathfrak{v}}\right\rangle}{\partial \mathbf{E}(\mathbf{R}, t)}-\left\langle\left.\frac{1}{c} \frac{\partial \delta \mathbf{A}(\mathfrak{x}, t)}{\partial t}\right|_{\mathfrak{x}} \cdot \frac{\widehat{\partial}_{(\mathfrak{x}, t)} \mathcal{T}}{\partial \mathbf{E}(\mathfrak{x}, t)}\right\rangle \\
& +\left(\nabla_{\mathbf{R}} \times \delta \mathbf{A}(\mathbf{R}, t)\right) \cdot \frac{\widehat{\partial}_{(\mathbf{R}, U, J ; t)}\langle\mathcal{T}\rangle}{\partial \mathbf{B}(\mathbf{R}, t)}+\left\langle\left(\nabla_{\mathfrak{x}} \times \delta \mathbf{A}(\mathfrak{x}, t)\right) \cdot \frac{\widehat{\partial}_{(\mathfrak{x}, t)} \mathcal{T}}{\partial \mathbf{B}(\mathfrak{x}, t)}\right\rangle \\
& +\left(\frac{\partial}{\partial R_{i}} \boldsymbol{\nabla}_{\mathbf{R}} \times \delta \mathbf{A}(\mathbf{R}, t)\right) \cdot \frac{\widehat{\partial}_{(\mathbf{R}, U, J ; t)}\langle\mathcal{T}\rangle}{\partial \mathbf{B}_{, R_{i}}(\mathbf{R}, t)} \\
& \left.+\left\langle\left(\frac{\partial}{\partial \mathbf{x}_{\mathfrak{i}}} \nabla_{\mathfrak{x}} \times \delta \mathbf{A}(\mathfrak{x}, t)\right) \cdot \frac{\widehat{\partial}_{(\mathfrak{x}, t)} \mathcal{T}}{\partial \mathbf{B}_{, \mathrm{x}_{\mathrm{i}}}(\mathfrak{x}, t)}\right\rangle\right\} \\
& -\frac{\partial}{\partial t}\left[\mathcal{B} f\left\langle\mathfrak{p} \cdot \delta_{\delta \mathbf{A}} \boldsymbol{\rho}\right\rangle\right]-\frac{\partial}{\partial \mathbf{R}} \cdot\left[\mathbf{V}_{\mathbf{R}} \mathcal{B} f\left\langle\mathfrak{p} \cdot \delta_{\delta \mathbf{A}} \boldsymbol{\rho}\right\rangle\right] \\
& -\frac{\partial}{\partial U}\left[V_{U} \mathcal{B} f\left\langle\mathfrak{p} \cdot \delta_{\delta \mathbf{A}} \boldsymbol{\rho}\right\rangle\right],
\end{aligned}
$$

where $\mathbf{B}_{, R_{i}}(\mathbf{R}, t)=\partial \mathbf{B}(\mathbf{R}, t) / \partial R_{i}, \mathbf{B}_{, \mathbf{x}_{\mathrm{i}}}(\mathfrak{x}, t)=\partial \mathbf{B}(\mathfrak{x}, t) / \partial \mathrm{x}_{\mathrm{i}}$, and summation with respect to the index $i$ is implied. The $\mathrm{x}_{\mathrm{i}}$ are the Cartesian components of the vector $\mathfrak{x}$. The quantity $\mathcal{T}=\mathcal{T}_{\mathfrak{v}}+\mathcal{T}_{\rho}$ is defined in (A10)-(A16). Equation (63) can be transformed by applying the same method which led to the charge density. Several terms which are important for the local conservation laws will not contribute, however, to the action integral, since they vanish after integration. The variational principle

$$
0=\delta_{\delta \mathrm{A}} \mathcal{A}_{\mathrm{tot} .}=\delta_{\delta \mathrm{A}} \mathcal{A}_{\mathrm{M}}+\delta_{\delta \mathrm{A}} \mathcal{A}_{\mathrm{K}}
$$




$$
\begin{aligned}
= & \int d t d^{3} r \frac{1}{c} \delta \mathbf{A} \cdot\left[\frac{1}{4 \pi} \frac{\partial \mathbf{E}(\mathbf{r}, t)}{\partial t}-\frac{c}{4 \pi} \nabla \times \mathbf{B}(\mathbf{r}, t)\right] \\
& -\sum_{\text {p. s. }} \int d t d^{3} R d U d J \mathcal{B} f\left(\delta_{\delta \mathbf{A}} H_{\mathrm{D}}\right)_{\text {constr. }}
\end{aligned}
$$

then yields after some steps the current density as a sum of particle-like, polarization and magnetization current densities:

$$
\begin{aligned}
\mathbf{j}(\mathbf{r}, t) & =\frac{c}{4 \pi} \nabla \times \mathbf{B}(\mathbf{r}, t)-\frac{1}{4 \pi} \frac{\partial \mathbf{E}(\mathbf{r}, t)}{\partial t} \\
& =\mathbf{j}_{\text {particle-like }}(\mathbf{r}, t)+\mathbf{j}_{\text {polarization }}(\mathbf{r}, t)+\mathbf{j}_{\text {magnetization }}(\mathbf{r}, t)
\end{aligned}
$$

with

$$
\begin{aligned}
& \mathbf{j}_{\text {particle-like }}=\sum_{\text {p. s. }} e \int d^{3} R d U d J \mathcal{B} f\left[\mathbf{V}_{\mathbf{R}}\langle\delta(\mathbf{r}-\mathfrak{x})\rangle+\left\langle\frac{\mathfrak{D} \boldsymbol{\rho}}{\mathfrak{D} t} \delta(\mathbf{r}-\mathfrak{x})\right\rangle\right] \\
& \mathbf{j}_{\text {polarization }}=-\frac{\partial}{\partial t} \sum_{\text {p. s. }} \int d^{3} R d U d J \mathcal{B} f\left[\delta(\mathbf{r}-\mathbf{R}) \frac{\widehat{\partial}_{(\mathbf{R}, U, J ; t)}\left\langle\mathcal{T}_{\mathfrak{v}}\right\rangle}{\partial \mathbf{E}(\mathbf{R}, t)}\right. \\
&\left.+\left\langle\delta(\mathbf{r}-\mathfrak{x}) \frac{\widehat{\partial}_{(\mathfrak{x}, t)} \mathcal{T}_{\mathfrak{v}}}{\partial \mathbf{E}(\mathfrak{x}, t)}\right\rangle\right] \\
&= \frac{\partial \mathbf{P}}{\partial t}
\end{aligned}
$$

and

$$
\mathbf{j}_{\text {magnetization }}=c \boldsymbol{\nabla}_{\mathbf{r}} \times \mathbf{M}, \quad \mathbf{M}=\mathbf{M}_{1}+\mathbf{M}_{2},
$$

where $\mathbf{M}=\mathbf{M}_{1}+\mathbf{M}_{2}$ is the magnetization, defined by the equations

$$
\mathbf{M}_{1} \equiv-\sum_{\text {p.s. }} \int d^{3} R d U d J \mathcal{B} f\left[\delta(\mathbf{r}-\mathbf{R}) \frac{\widehat{\partial}_{(\mathbf{R}, U, J ; t)}\langle\mathcal{T}\rangle}{\partial \mathbf{B}(\mathbf{R}, t)}+\left\langle\delta(\mathbf{r}-\mathfrak{x}) \frac{\widehat{\partial}_{(\mathfrak{x}, t)} \mathcal{T}_{\mathfrak{v}}}{\partial \mathbf{B}(\mathfrak{x}, t)}\right\rangle\right]
$$

and

$$
\begin{gathered}
\mathbf{M}_{2} \equiv \frac{\partial}{\partial r_{i}} \mathcal{M}_{i} \\
\mathcal{M}_{i} \equiv \sum_{\text {p.s. }} \int d^{3} R d U d J \mathcal{B} f\left[\delta(\mathbf{r}-\mathbf{R}) \frac{\widehat{\partial}_{(\mathbf{R}, U, J ; t)}\langle\mathcal{T}\rangle}{\partial \mathbf{B}_{, R_{i}}(\mathbf{R}, t)}\right. \\
\left.+\left\langle\delta(\mathbf{r}-\mathfrak{x}) \frac{\widehat{\partial}_{(\mathfrak{x}, t)} \mathcal{T}_{\mathfrak{v}}}{\partial \mathbf{B}_{, \mathrm{x}_{\mathrm{i}}}(\mathfrak{x}, t)}\right\rangle\right]
\end{gathered}
$$

Summation with respect to $i=1,2,3$ is implied in (70) and (A19) and (A20) were used in the transformations. 


\section{Local conservation laws}

\subsection{Conservation of the density of gyration centres}

Integration of (44) with respect to $\alpha$, with the Dirac constraints taken into account according to (35), yields for $\mathcal{B}(\mathbf{R}, U, J ; t) f(\mathbf{R}, U, J ; t)$ the equation

$$
\frac{\partial}{\partial t}(\mathcal{B} f)+\frac{\partial}{\partial \mathbf{R}} \cdot\left(\mathbf{V}_{\mathbf{R}} \mathcal{B} f\right)+\frac{\partial}{\partial U}\left(V_{U} \mathcal{B} f\right)=0
$$

Integration of this equation in velocity space, i.e with respect to $U$ and $J$, yields the conservation law for the density of each species:

$$
\frac{\partial}{\partial t} \int d U d J \mathcal{B} f+\frac{\partial}{\partial \mathbf{R}} \cdot \int d U d J \mathbf{V}_{\mathbf{R}} \mathcal{B} f=0
$$

\subsection{Charge conservation}

It will be shown, on the basis of (59)-(62) and (65)-(71), that the electric charge is locally conserved, i.e.

$$
\frac{\partial \rho(\mathbf{r}, t)}{\partial t}+\nabla \cdot \mathbf{j}(\mathbf{r}, t)=0
$$

which is valid for the total charge and current densities, but also separately for the particle-like, electric polarization and magnetization contributions (the latter without a counterpart in the charge density). While the conservation law is evident for the polarization and magnetization contributions, it must be proved for the particle-like charge and current densities.

Multiplication of $(72)$ by $\delta(\mathbf{r}-\mathfrak{x}(\mathbf{R}, U, J, \phi ; t))$ and integration with respect to $\mathbf{R}, U, J$ and $\phi$, with the vanishing of some terms at the boundaries of $\mathbf{R}$ and $U$ taken into account, yields

$$
\begin{aligned}
& \left.\frac{\partial}{\partial t}\right|_{\mathbf{r}} \int d^{3} R d U d J \mathcal{B} f\langle\delta(\mathbf{r}-\mathfrak{x})\rangle \\
& =\int d^{3} R d U d J d \phi \mathcal{B} f\left[\frac{\partial \delta(\mathbf{r}-\mathfrak{x})}{\partial t}+\mathbf{V}_{\mathbf{R}} \cdot \frac{\partial \delta(\mathbf{r}-\mathfrak{x})}{\partial \mathbf{R}}+V_{U} \frac{\partial \delta(\mathbf{r}-\mathfrak{x})}{\partial U}\right] \\
& =\int d^{3} R d U d J d \phi \mathcal{B} f \frac{\mathfrak{D} \delta(\mathbf{r}-\mathfrak{x})}{\mathfrak{D} t} \\
& =-\left.\frac{\partial}{\partial \mathbf{r}}\right|_{t} \cdot \int d^{3} R d U d J \mathcal{B} f\left[\mathbf{V}_{\mathbf{R}}\langle\delta(\mathbf{r}-\mathfrak{x})\rangle+\left\langle\frac{\mathfrak{D} \boldsymbol{\rho}}{\mathfrak{D} t} \delta(\mathbf{r}-\mathfrak{x})\right\rangle\right],
\end{aligned}
$$

where the time derivative of $\delta(\mathbf{r}-\mathfrak{x})$ was transformed according to the relation $\mathfrak{D} \delta(\mathbf{r}-\mathfrak{x}) / \mathfrak{D} t=\mathfrak{D} \mathfrak{x} / \mathfrak{D} t \cdot \partial \delta(\mathbf{r}-\mathfrak{x}) / \partial \mathfrak{x}=-\mathfrak{D} \mathfrak{x} / \mathfrak{D} t \cdot \partial \delta(\mathbf{r}-\mathfrak{x}) / \partial \mathbf{r}$, and $\mathfrak{D} \mathfrak{x} / \mathfrak{D} t=$ $\mathbf{V}_{\mathbf{R}}+\mathfrak{D} \boldsymbol{\rho} / \mathfrak{D} t$ was inserted. Multiplication of (75) by the charge $e$ and summation over the particle species then yields the conservation law

$$
\frac{\partial}{\partial t} \rho_{\text {particle-like }}(\mathbf{r}, t)+\nabla \cdot \mathbf{j}_{\text {particle-like }}(\mathbf{r}, t)=0 .
$$




\subsection{Local energy, momentum and angular momentum conservation}

Derivation of local energy and momentum conservation laws by means of Noether's procedure requires an expression for the total Lagrange density, and one has to consider the kinetic Lagrange density at the same space-time point $(\mathbf{r}, t)$ as appears in the Lagrange density of the Maxwell part. There is an infinitely large manifold of equivalent definitions for the Lagrange density of the kinetic part. Within the framework of Kruskal's theory, it is most natural to identify the gyrocentre position $\mathbf{R}$ with the point $\mathbf{r}$. As shown in $[1,2], \mathbf{R}$ can be defined in any finite-order Kruskal theory, but not in an exact theory. The latter feature reflects the fact that Kruskal's theory does not yield a convergent series, in agreement with the fact that the adiabatic invariants are not exact constants of motion.

The Lagrange density $\mathcal{L}_{\mathrm{K}}(\mathbf{r}, t)$ for the kinetic part of the whole theory then follows from (3) and the expression

$$
\mathcal{A}_{\mathrm{K}}=\int d t d^{3} r \mathcal{L}_{\mathrm{K}}(\mathbf{r}, t)
$$

as

$$
\mathcal{L}_{\mathrm{K}}(\mathbf{r}, t)=-\sum_{\text {p. s. }} \int d^{3} R d U d^{4} \alpha d J \delta(\mathbf{r}-\mathbf{R}) f_{\mathrm{p}}\left[\frac{\partial S}{\partial t}+H_{\mathrm{D} S}\right] .
$$

The variation of this density owing to variations of $f_{\mathrm{p}}, S$ and the potentials $\Phi$ and $\mathbf{A}$ can be written as

$$
\delta \mathcal{L}_{\mathrm{K}}(\mathbf{r}, t)=\delta_{\delta f_{\mathrm{p}}} \mathcal{L}_{\mathrm{K}}(\mathbf{r}, t)+\delta_{\delta S} \mathcal{L}_{\mathrm{K}}(\mathbf{r}, t)+\delta_{\delta_{\mathrm{pot} .}} \mathcal{L}_{\mathrm{K}}(\mathbf{r}, t)
$$

The contribution of $\delta f_{\mathrm{p}}$ is

$$
\delta_{\delta f_{\mathrm{p}}} \mathcal{L}_{\mathrm{K}}(\mathbf{r}, t)=-\sum_{\mathrm{p} . \mathrm{s} .} \int d^{3} R d U d^{4} \alpha d J \delta(\mathbf{r}-\mathbf{R}) \delta f_{\mathrm{p}}\left[\frac{\partial S}{\partial t}+H_{\mathrm{D} S}\right] .
$$

After inserting the Euler-Lagrange equations for $S$, i.e. the Hamilton-Jacobi equations, this expression vanishes:

$$
\delta_{\delta f_{\mathrm{p}}}^{\mathrm{EL}} \mathcal{L}_{\mathrm{K}}(\mathbf{r}, t)=0
$$

Here, the superscript E L means that the Euler-Lagrange equations were used. The variation of $S$ yields

$$
\begin{aligned}
\delta_{\delta S} \mathcal{L}_{\mathrm{K}}(\mathbf{r}, t)=-\sum_{\mathrm{p} . \mathrm{s} .} \int d^{3} R d U d^{4} \alpha d J \delta(\mathbf{r}-\mathbf{R}) f_{\mathrm{p}} \\
\times \\
\times\left[\frac{\partial \delta S}{\partial t}+\mathbf{V}_{\mathbf{R}} \cdot \frac{\partial \delta S}{\partial \mathbf{R}}+V_{U} \frac{\partial \delta S}{\partial U}\right] \\
=-\sum_{\text {p. s. }} \int d^{3} R d U d^{4} \alpha d J\left[\frac{\partial}{\partial t}\left[\delta(\mathbf{r}-\mathbf{R}) f_{\mathrm{p}} \delta S\right]-f_{\mathrm{p}} \delta S \mathbf{V}_{\mathbf{R}} \cdot \frac{\partial}{\partial \mathbf{R}} \delta(\mathbf{r}-\mathbf{R})\right.
\end{aligned}
$$




$$
\begin{aligned}
& +\frac{\partial}{\partial \mathbf{R}} \cdot\left[\delta(\mathbf{r}-\mathbf{R}) \mathbf{V}_{\mathbf{R}} f_{\mathrm{p}} \delta S\right]+\frac{\partial}{\partial U}\left[\delta(\mathbf{r}-\mathbf{R}) V_{U} f_{\mathrm{p}} \delta S\right] \\
& \left.-\delta(\mathbf{r}-\mathbf{R}) \delta S\left[\frac{\partial f_{\mathrm{p}}}{\partial t}+\frac{\partial}{\partial \mathbf{R}} \cdot\left(\mathbf{V}_{\mathbf{R}} f_{\mathrm{p}}\right)+\frac{\partial}{\partial U}\left(V_{U} f_{\mathrm{p}}\right)\right]\right] .
\end{aligned}
$$

The terms in brackets in the last line of this equation vanish after inserting the Euler-Lagrange equations for $f_{\mathrm{p}},(44)$. The two terms which are divergences in $\mathbf{R}$ and $U$, respectively, vanish at the boundaries after integration. After transformation from $\alpha_{i}$ and $f_{\mathrm{p}}$ to $\mathbf{P}_{\mathbf{R}}, P_{U}$ and $f$, with the Dirac constraints taken into account in the usual way, one obtains

$$
\begin{aligned}
\delta_{\delta S}^{\mathrm{EL}} \mathcal{L}_{\mathrm{K}}(\mathbf{r}, t)= & -\sum_{\text {p. s. }} \frac{\partial}{\partial t} \int d U d J\left[\mathcal{B} f(\delta S)_{\text {constr. }}\right]_{\mathbf{R}=\mathbf{r}} \\
& -\sum_{\text {p. s. }} \frac{\partial}{\partial \mathbf{r}} \cdot \int d U d J\left[\mathcal{B} f \mathbf{V}_{\mathbf{R}}(\delta S)_{\text {constr. }}\right]_{\mathbf{R}=\mathbf{r}} .
\end{aligned}
$$

The variation of $\mathcal{L}_{\mathrm{K}}$ owing to $\delta \Phi$ is

$$
\begin{aligned}
\delta_{\delta \Phi} \mathcal{L}_{\mathrm{K}}(\mathbf{r}, t)= & -\sum_{\mathrm{p} . \mathrm{s.}} \int d^{3} R d U d^{4} \alpha d J \delta(\mathbf{r}-\mathbf{R}) f_{\mathrm{p}} \delta_{\delta \Phi} H_{\mathrm{D} S} \\
= & -\sum_{\text {p.s. }} \int d^{3} R d U d J \mathcal{B} \delta(\mathbf{r}-\mathbf{R}) f\left(\delta_{\delta \Phi} H_{\mathrm{D}}\right)_{\text {constr. }} \\
= & -\sum_{\text {p.s. }} \int d^{3} R d U d J \mathcal{B} \delta(\mathbf{r}-\mathbf{R}) f[e\langle\delta \Phi(\mathfrak{x}, t)\rangle \\
& \left.-\frac{\partial \delta \Phi(\mathbf{R}, t)}{\partial \mathbf{R}} \cdot \frac{\widehat{\partial}_{(\mathbf{R}, U, J ; t)}\left\langle\mathcal{T}_{\mathfrak{v}}\right\rangle}{\partial \mathbf{E}(\mathbf{R}, t)}-\left\langle\frac{\partial \delta \Phi(\mathfrak{x}, t)}{\partial \mathfrak{x}} \cdot \frac{\widehat{\partial}_{(\mathfrak{x}, t)} \mathcal{T}_{\mathfrak{v}}}{\partial \mathbf{E}(\mathfrak{x}, t)}\right\rangle\right],
\end{aligned}
$$

where (55) was used. After some trivial transformations, and with the results of (B1) and (B2), one obtains

$$
\begin{aligned}
\delta_{\delta \Phi} \mathcal{L}_{\mathrm{K}}(\mathbf{r}, t)=-\sum_{\mathbf{p}, \mathbf{s} .} \int d^{3} R d U d J[\delta \Phi(\mathbf{r}, t) e \mathcal{B} f\langle\delta(\mathbf{r}-\mathfrak{x})\rangle \\
+e \mathcal{B} f\langle(\delta(\mathbf{r}-\mathbf{R})-\delta(\mathbf{r}-\mathfrak{x})) \delta \Phi(\mathfrak{x}, t)\rangle \\
\quad-\frac{\partial}{\partial \mathbf{r}} \cdot\left[\delta(\mathbf{r}-\mathbf{R}) \delta \Phi(\mathbf{r}, t) \mathcal{B} f \frac{\widehat{\partial}_{(\mathbf{R}, U, J ; t)}\left\langle\mathcal{T}_{\mathfrak{v}}\right\rangle}{\partial \mathbf{E}(\mathbf{R}, t)}\right] \\
+\delta \Phi(\mathbf{r}, t) \frac{\partial}{\partial \mathbf{r}} \cdot\left[\delta(\mathbf{r}-\mathbf{R}) \mathcal{B} f \frac{\widehat{\partial}_{(\mathbf{R}, U, J ; t)}\left\langle\mathcal{T}_{\mathfrak{v}}\right\rangle}{\partial \mathbf{E}(\mathbf{R}, t)}\right] \\
-\frac{\partial}{\partial \mathbf{r}} \cdot\left[\delta \Phi(\mathbf{r}, t) \mathcal{B} f\left\langle\delta(\mathbf{r}-\mathfrak{x}) \frac{\widehat{\partial}_{(\mathfrak{x}, t)} \mathcal{T}_{\mathfrak{v}}}{\partial \mathbf{E}(\mathfrak{x}, t)}\right\rangle\right] \\
+\delta \Phi(\mathbf{r}, t) \frac{\partial}{\partial \mathbf{r}} \cdot\left[\mathcal{B} f\left\langle\delta(\mathbf{r}-\mathfrak{x}) \frac{\widehat{\partial}_{(\mathfrak{x}, t)} \mathcal{T}_{\mathfrak{v}}}{\partial \mathbf{E}(\mathfrak{x}, t)}\right\rangle\right] \\
\left.-\mathcal{B} f\left\langle(\delta(\mathbf{r}-\mathbf{R})-\delta(\mathbf{r}-\mathfrak{x})) \frac{\partial \delta \Phi(\mathfrak{x}, t)}{\partial \mathfrak{x}} \cdot \frac{\widehat{\partial}_{(\mathfrak{x}, t)} \mathcal{T}_{\mathfrak{v}}}{\partial \mathbf{E}(\mathfrak{x}, t)}\right\rangle\right] .
\end{aligned}
$$


After inserting the Euler-Lagrange equations for $\Phi$, i.e. the equations for the electric charge density, (59)-(62), one obtains

$$
\begin{aligned}
\delta_{\delta \Phi}^{\mathrm{EL}} & \mathcal{L}_{\mathrm{K}}(\mathbf{r}, t)=-\delta \Phi(\mathbf{r}, t) \frac{1}{4 \pi} \boldsymbol{\nabla} \cdot \mathbf{E}(\mathbf{r}, t)-\boldsymbol{\nabla} \cdot[\delta \Phi(\mathbf{r}, t) \mathbf{P}(\mathbf{r}, t)] \\
\quad & -\sum_{\mathrm{p.} \mathrm{s.}} \int d^{3} R d U d J \mathcal{B} f \\
\quad & {\left[\left\langle(\delta(\mathbf{r}-\mathbf{R})-\delta(\mathbf{r}-\mathfrak{x}))\left[e \delta \Phi(\mathfrak{x}, t)-\frac{\partial \delta \Phi(\mathfrak{x}, t)}{\partial \mathfrak{x}} \cdot \frac{\widehat{\partial}_{(\mathfrak{x}, t)} \mathcal{T}_{\mathfrak{v}}}{\partial \mathbf{E}(\mathfrak{x}, t)}\right]\right\rangle\right] . }
\end{aligned}
$$

Note that the last term in this equation, like a divergence, has the property of vanishing after $\mathbf{r}$-integration. It represents a finite Larmor radius effect contribution to the local conservation laws. It will appear in the gyrokinetic theory, but not in the drift-kinetic theory.

The variation of $\mathcal{L}_{\mathrm{K}}$ owing to $\delta \mathbf{A}$ is

$$
\begin{aligned}
\delta_{\delta \mathbf{A}} \mathcal{L}_{\mathrm{K}}(\mathbf{r}, t)= & -\sum_{\mathrm{p.s.}} \int d^{3} R d U d^{4} \alpha d J \delta(\mathbf{r}-\mathbf{R}) f_{\mathrm{p}} \delta_{\delta \mathbf{A}} H_{\mathrm{D} S} \\
= & -\sum_{\text {p. s. }} \int d^{3} R d U d J \mathcal{B} \delta(\mathbf{r}-\mathbf{R}) f\left(\delta_{\delta \mathbf{A}} H_{\mathrm{D}}\right)_{\text {constr. }} \\
= & \sum_{\text {p.s. }} \int d^{3} R d U d J \delta(\mathbf{r}-\mathbf{R}) \\
& \times\left[\mathcal { B } f \left\{\frac{e}{c} \mathbf{V}_{\mathbf{R}} \cdot\langle\delta \mathbf{A}(\mathfrak{x}, t)\rangle+\frac{e}{c}\left\langle\frac{\mathfrak{D} \boldsymbol{\rho}}{\mathfrak{D} t} \cdot \delta \mathbf{A}(\mathfrak{x}, t)\right\rangle\right.\right. \\
& +\left.\frac{1}{c} \frac{\partial \delta \mathbf{A}(\mathbf{R}, t)}{\partial t}\right|_{\mathbf{R}} \cdot \frac{\widehat{\partial}_{(\mathbf{R}, U, J ; t)}\left\langle\mathcal{T}_{\mathfrak{v}}\right\rangle}{\partial \mathbf{E}(\mathbf{R}, t)}+\left\langle\left.\frac{1}{c} \frac{\partial \delta \mathbf{A}(\mathfrak{x}, t)}{\partial t}\right|_{\mathfrak{x}} \cdot \frac{\widehat{\partial}_{(\mathfrak{x}, t)} \mathcal{T}_{\mathfrak{v}}}{\partial \mathbf{E}(\mathfrak{x}, t)}\right\rangle \\
& -(\boldsymbol{\nabla} \mathbf{R} \times \delta \mathbf{A}(\mathbf{R}, t)) \cdot \frac{\widehat{\partial}_{(\mathbf{R}, U, J ; t)}\langle\mathcal{T}\rangle}{\partial \mathbf{B}(\mathbf{R}, t)}-\left\langle\left(\boldsymbol{\nabla}_{\mathfrak{x}} \times \delta \mathbf{A}(\mathfrak{x}, t)\right) \cdot \frac{\widehat{\partial}_{(\mathfrak{x}, t)} \mathcal{T}_{\mathfrak{v}}}{\partial \mathbf{B}(\mathfrak{x}, t)}\right\rangle \\
& -\left(\frac{\partial}{\partial R_{i}} \nabla_{\mathbf{R}} \times \delta \mathbf{A}(\mathbf{R}, t)\right) \cdot \frac{\widehat{\partial}_{(\mathbf{R}, U, J ; t)}\langle\mathcal{T}\rangle}{\partial \mathbf{B}_{, R_{i}}(\mathbf{R}, t)} \\
& \left.-\left\langle\left(\frac{\partial}{\partial \mathrm{x}_{\mathfrak{i}}} \nabla_{\mathfrak{x}} \times \delta \mathbf{A}(\mathfrak{x}, t)\right) \cdot \frac{\widehat{\partial}_{(\mathfrak{x}, t)} \mathcal{T}_{\mathfrak{v}}}{\partial \mathbf{B}_{, \mathrm{x}_{\mathrm{i}}}(\mathfrak{x}, t)}\right\rangle\right\} \\
& \left.+\frac{\partial}{\partial t}\left[\mathcal{B} f\left\langle\mathfrak{p} \cdot \delta_{\delta \mathbf{A}} \boldsymbol{\rho}\right\rangle\right]+\frac{\partial}{\partial \mathbf{R}} \cdot\left[\mathbf{V}_{\mathbf{R}} \mathcal{B} f\left\langle\mathfrak{p} \cdot \delta_{\delta \mathbf{A}} \boldsymbol{\rho}\right\rangle\right]\right]
\end{aligned}
$$

where (A19) and (A20) were used, and (63) was inserted, with its last term vanishing after $U$-integration. The equation for $\delta_{\delta \mathbf{A}} \mathcal{L}_{\mathrm{K}}(\mathbf{r}, t)$ can be transformed by taking into account (B3)-(B8) and the Euler-Lagrange equations for A, i.e the equations for the current density, (65)-(71). One obtains

$$
\delta_{\delta \mathbf{A}}^{\mathrm{EL}} \mathcal{L}_{\mathrm{K}}(\mathbf{r}, t)=\delta \mathbf{A}(\mathbf{r}, t) \cdot\left[\frac{1}{4 \pi} \boldsymbol{\nabla} \times \mathbf{B}(\mathbf{r}, t)-\frac{1}{4 \pi c} \frac{\partial \mathbf{E}(\mathbf{r}, t)}{\partial t}\right]
$$




$$
\begin{aligned}
& +\left.\sum_{\text {p. s. }} \frac{\partial}{\partial t}\right|_{\mathbf{r}} \int d^{3} R d U d J \mathcal{B} f \\
& \times \frac{1}{c} \delta \mathbf{A}(\mathbf{r}, t) \cdot\left[\delta(\mathbf{r}-\mathbf{R}) \frac{\widehat{\partial}_{(\mathbf{R}, U, J ; t)}\left\langle\mathcal{T}_{\mathfrak{v}}\right\rangle}{\partial \mathbf{E}(\mathbf{R}, t)}+\left\langle\delta(\mathbf{r}-\mathfrak{x}) \frac{\widehat{\partial}_{(\mathfrak{x}, t)} \mathcal{T}_{\mathfrak{v}}}{\partial \mathbf{E}(\mathfrak{x}, t)}\right\rangle\right] \\
& -\sum_{\text {p. s. }} \frac{\partial}{\partial \mathbf{r}} \cdot \int d^{3} R d U d J \mathcal{B} f \\
& \times\left[\delta \mathbf{A}(\mathbf{r}, t) \times\left[\delta(\mathbf{r}-\mathbf{R}) \frac{\widehat{\partial}_{(\mathbf{R}, U, J ; t)}\langle\mathcal{T}\rangle}{\partial \mathbf{B}(\mathbf{R}, t)}-\frac{\partial}{\partial r_{i}}\left[\delta(\mathbf{r}-\mathbf{R}) \frac{\widehat{\partial}_{(\mathbf{R}, U, J ; t)}\langle\mathcal{T}\rangle}{\partial \mathbf{B}_{, R_{i}}(\mathbf{R}, t)}\right]\right]\right. \\
& \left.+\delta \mathbf{A}(\mathbf{r}, t) \times\left\langle\delta(\mathbf{r}-\mathfrak{x}) \frac{\widehat{\partial}_{(\mathfrak{x}, t)} \mathcal{T}_{\mathfrak{v}}}{\partial \mathbf{B}(\mathfrak{x}, t)}-\frac{\partial}{\partial r_{i}}\left[\delta(\mathbf{r}-\mathfrak{x}) \frac{\widehat{\partial}_{(\mathfrak{x}, t)} \mathcal{T}_{\mathfrak{v}}}{\partial \mathbf{B}_{\mathrm{x}_{\mathrm{i}}}(\mathfrak{x}, t)}\right]\right\rangle\right] \\
& -\sum_{\text {p.s. }} \frac{\partial}{\partial r_{i}} \int d^{3} R d U d J \mathcal{B} f \\
& \times\left[\delta \mathbf{B}(\mathbf{r}, t) \cdot\left[\delta(\mathbf{r}-\mathbf{R}) \frac{\widehat{\partial}_{(\mathbf{R}, U, J ; t)}\langle\mathcal{T}\rangle}{\partial \mathbf{B}_{, R_{i}}(\mathbf{R}, t)}+\left\langle\delta(\mathbf{r}-\mathfrak{x}) \frac{\widehat{\partial}_{(\mathfrak{x}, t)} \mathcal{T}_{\mathfrak{v}}}{\partial \mathbf{B}_{, \mathrm{x}_{\mathrm{i}}}(\mathfrak{x}, t)}\right\rangle\right]\right] \\
& +\sum_{\text {p. s. }} \int d^{3} R d U d J \mathcal{B} f\langle(\delta(\mathbf{r}-\mathbf{R})-\delta(\mathbf{r}-\mathfrak{x})) \\
& \times\left[\frac{e}{c} \delta \mathbf{A}(\mathfrak{x}, t) \cdot\left[\mathbf{V}_{\mathbf{R}}+\frac{\mathfrak{D} \boldsymbol{\rho}}{\mathfrak{D} t}\right]+\left.\frac{1}{c} \frac{\partial \delta \mathbf{A}(\mathfrak{x}, t)}{\partial t}\right|_{\mathfrak{x}} \cdot \frac{\widehat{\partial}_{(\mathfrak{x}, t)} \mathcal{T}_{\mathfrak{v}}}{\partial \mathbf{E}(\mathfrak{x}, t)}\right. \\
& \left.\left.-\delta \mathbf{B}(\mathfrak{x}, t) \cdot \frac{\widehat{\partial}_{(x, t)} \mathcal{T}_{\mathfrak{v}}}{\partial \mathbf{B}(\mathfrak{x}, t)}-\left(\frac{\partial \delta \mathbf{B}(\mathfrak{x}, t)}{\partial \mathrm{x}_{\mathrm{i}}}\right) \cdot \frac{\widehat{\partial}_{(x, t)} \mathcal{T}_{\mathfrak{v}}}{\partial \mathbf{B}_{, \mathrm{x}_{\mathrm{i}}}(\mathfrak{x}, t)}\right]\right\rangle \\
& +\sum_{\text {p. s. }} \frac{\partial}{\partial t} \int d U d J\left[\mathcal{B} f\left\langle\mathfrak{p} \cdot \delta_{\delta \mathbf{A}} \boldsymbol{\rho}\right\rangle\right]_{\mathbf{R}=\mathbf{r}}+\sum_{\text {p.s. }} \frac{\partial}{\partial \mathbf{r}} \cdot \int d U d J\left[\mathbf{V}_{\mathbf{R}} \mathcal{B} f\left\langle\mathfrak{p} \cdot \delta_{\delta \mathbf{A}} \boldsymbol{\rho}\right\rangle\right]_{\mathbf{R}=\mathbf{r}}
\end{aligned}
$$

This equation can be simplified when (62) and (68)-(71) for the polarazation $\mathbf{P}$ and the magnetization $\mathbf{M}$ are taken into account:

$$
\begin{aligned}
\delta_{\delta \mathbf{A}}^{\mathrm{EL}} \mathcal{L}_{\mathrm{K}}(\mathbf{r}, t)=\delta \mathbf{A}(\mathbf{r}, t) \cdot\left[\frac{1}{4 \pi} \mathbf{\nabla} \times \mathbf{B}(\mathbf{r}, t)-\frac{1}{4 \pi c} \frac{\partial \mathbf{E}(\mathbf{r}, t)}{\partial t}\right] \\
-\frac{\partial}{\partial t}\left[\delta \mathbf{A}(\mathbf{r}, t) \cdot \frac{1}{c} \mathbf{P}(\mathbf{r}, t)\right]+\frac{\partial}{\partial \mathbf{r}} \cdot[\delta \mathbf{A}(\mathbf{r}, t) \times \mathbf{M}(\mathbf{r}, t)] \\
-\quad \frac{\partial}{\partial r_{i}}\left[\delta \mathbf{B}(\mathbf{r}, t) \cdot \mathcal{M}_{i}\right] \\
+\sum_{\mathrm{p.s.}} \int d^{3} R d U d J \mathcal{B} f\langle(\delta(\mathbf{r}-\mathbf{R})-\delta(\mathbf{r}-\mathfrak{x})) \\
\quad\left[\frac{e}{c} \delta \mathbf{A}(\mathfrak{x}, t) \cdot\left[\mathbf{V}_{\mathbf{R}}+\frac{\mathfrak{D} \boldsymbol{\rho}}{\mathfrak{D} t}\right]+\left.\frac{1}{c} \frac{\partial \delta \mathbf{A}(\mathfrak{x}, t)}{\partial t}\right|_{\mathfrak{x}} \cdot \frac{\widehat{\partial}_{(\mathfrak{x}, t)}}{\partial \mathbf{E}(\mathfrak{x}, t)}\right. \\
\left.\left.\quad-\delta \mathbf{B}(\mathfrak{x}, t) \cdot \frac{\widehat{\partial}_{(\mathfrak{v}, t)}}{\partial \mathbf{B}(\mathfrak{x}, t)}-\left(\frac{\partial \delta \mathbf{B}(\mathfrak{x}, t)}{\partial \mathrm{x}_{\mathrm{i}}}\right) \cdot \frac{\widehat{\partial}_{(\mathfrak{x}, t)}}{\partial \mathcal{T}_{\mathfrak{v}}}\right]\right\rangle
\end{aligned}
$$


$+\sum_{\text {p. s. }} \frac{\partial}{\partial t} \int d U d J\left[\mathcal{B} f\left\langle\mathfrak{p} \cdot \delta_{\delta \mathbf{A}} \boldsymbol{\rho}\right\rangle\right]_{\mathbf{R}=\mathbf{r}}+\sum_{\text {p.s. }} \frac{\partial}{\partial \mathbf{r}} \cdot \int d U d J\left[\mathbf{V}_{\mathbf{R}} \mathcal{B} f\left\langle\mathfrak{p} \cdot \delta_{\delta \mathbf{A}} \boldsymbol{\rho}\right\rangle\right]_{\mathbf{R}=\mathbf{r}}$

The quantity $\delta_{\delta \mathbf{A}} \boldsymbol{\rho}$, which appears in the last two terms, is given by (51). As will be seen later, these terms combine with contributions from $\delta S,(83)$, to form an expression which can be made gauge invariant.

\subsubsection{Local energy conservation}

The local energy conservation law is obtained by means of the usual Noether procedure, but using a particularly appropriate, non-standard kind of variations. As explained in [14], we take the variations to be the gauge-invariant result of a shift plus an infinitesimal gauge transformation, as given in Appendix $\mathrm{C}$, instead of taking the usual non-gauge-invariant shift variations of $S, \Phi$ and $\mathbf{A}$. As a consequence of this, the expressions entering the conservation laws are immediately gauge invariant, as they should be.

Owing to a time shift $\epsilon_{t}$, the Euler variation of the total Lagrange density is

$$
\delta \mathcal{L}_{\text {tot. }}(\mathbf{r}, t)=-\epsilon_{t} \frac{\partial}{\partial t} \mathcal{L}_{\text {tot. }}(\mathbf{r}, t)
$$

For the actual development of the system, the kinetic Lagrange density $\mathcal{L}_{\mathrm{K}}(\mathbf{r}, t)$ does not contribute, because it vanishes after inserting the Euler-Lagrange equations for $S$, i.e. the Hamilton-Jacobi equations, (42). One thus has

$$
\begin{aligned}
\delta \mathcal{L}_{\text {tot. }}(\mathbf{r}, t) & =\delta^{\mathrm{EL}} \mathcal{L}_{\text {tot. }}(\mathbf{r}, t)=\delta \mathcal{L}_{\mathrm{M}}(\mathbf{r}, t)=-\epsilon_{t} \frac{\partial}{\partial t} \mathcal{L}_{\mathrm{M}}(\mathbf{r}, t) \\
& =-\epsilon_{t} \frac{\partial}{\partial t}\left[\frac{1}{8 \pi}\left[\mathbf{E}^{2}(\mathbf{r}, t)-\mathbf{B}^{2}(\mathbf{r}, t)\right]\right]
\end{aligned}
$$

where, again, the superscript EL means that the Euler-Lagrange equations were used. On the other hand, one has the relation

$$
\begin{aligned}
\delta \mathcal{L}_{\text {tot. }}(\mathbf{r}, t)= & \delta \mathcal{L}_{\mathrm{M}}(\mathbf{r}, t)+\delta \mathcal{L}_{\mathrm{K}}(\mathbf{r}, t) \\
= & \delta \mathcal{L}_{\mathrm{M}}(\mathbf{r}, t)+\delta_{\delta f_{\mathrm{p}}} \mathcal{L}_{\mathrm{K}}(\mathbf{r}, t)+\delta_{\delta S} \mathcal{L}_{\mathrm{K}}(\mathbf{r}, t) \\
& +\delta_{\delta \Phi} \mathcal{L}_{\mathrm{K}}(\mathbf{r}, t)+\delta_{\delta \mathbf{A}} \mathcal{L}_{\mathrm{K}}(\mathbf{r}, t) .
\end{aligned}
$$

After inserting the Euler-Lagrange equations for $S, f_{\mathrm{p}}, \Phi$ and $\mathbf{A}$, one obtains

$$
\delta \mathcal{L}_{\text {tot. }}(\mathbf{r}, t)=\delta \mathcal{L}_{\mathrm{M}}(\mathbf{r}, t)+\delta_{\delta S}^{\mathrm{EL}} \mathcal{L}_{\mathrm{K}}(\mathbf{r}, t)+\delta_{\delta \Phi}^{\mathrm{EL}} \mathcal{L}_{\mathrm{K}}(\mathbf{r}, t)+\delta_{\delta \mathbf{A}}^{\mathrm{EL}} \mathcal{L}_{\mathrm{K}}(\mathbf{r}, t)
$$

with

$$
\begin{array}{r}
\delta \mathcal{L}_{\mathrm{M}}(\mathbf{r}, t)=\delta \Phi \frac{1}{4 \pi} \boldsymbol{\nabla} \cdot \mathbf{E}+\delta \mathbf{A} \cdot\left[\frac{1}{4 \pi c} \frac{\partial \mathbf{E}}{\partial t}-\frac{1}{4 \pi} \boldsymbol{\nabla} \times \mathbf{B}\right] \\
-\boldsymbol{\nabla} \cdot\left[\frac{1}{4 \pi} \delta \Phi \mathbf{E}\right]-\frac{1}{4 \pi c} \frac{\partial}{\partial t}[\delta \mathbf{A} \cdot \mathbf{E}]-\boldsymbol{\nabla} \cdot\left[\frac{1}{4 \pi} \delta \mathbf{A} \times \mathbf{B}\right]
\end{array}
$$




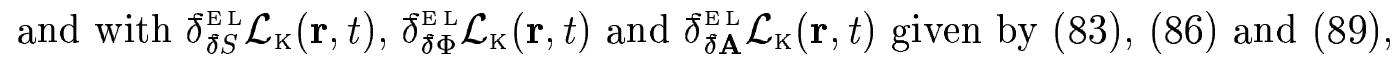
respectively. The term $\delta_{\delta f_{\mathrm{p}}}^{\mathrm{EL}} \mathcal{L}_{\mathrm{K}}(\mathbf{r}, t)$ does not contribute, since it vanishes owing to the Hamilton-Jacobi equations.

Following a displacement $\epsilon_{t}$ in time and a simultaneous infinitesimal gauge transformation, the variations of $S, \Phi$ and $\mathbf{A}$ are, according to (C31) and (C22)-(C25),

$$
\begin{gathered}
\left(\delta_{\text {comb. }} S\right)_{\text {constr. }}+\epsilon_{t}\left\langle\frac{\partial \boldsymbol{\rho}}{\partial t} \cdot \mathfrak{p}\right\rangle=\epsilon_{t}\left\langle\frac{m}{2} \mathfrak{v}^{2}\right\rangle \\
\delta_{\text {comb. }} \Phi=0 \quad, \quad \delta_{\text {comb. }} \mathbf{A}=\epsilon_{t} c \mathbf{E}
\end{gathered}
$$

By setting the right-hand side of (91) equal to that of (93), one obtains the local energy conservation law at the space-time point $(\mathbf{r}, t)$,

$$
\begin{aligned}
& \frac{\partial}{\partial t}\left[\frac{1}{8 \pi}\left[\mathbf{E}^{2}+\mathbf{B}^{2}\right]\right]+\frac{\partial}{\partial t} \sum_{\text {p.s. }} \int d U d J\left[\mathcal{B} f\left\langle\frac{m}{2} \mathfrak{v}^{2}\right\rangle\right]_{\mathbf{R}=\mathbf{r}} \\
& +\frac{\partial}{\partial t}[\mathbf{E} \cdot \mathbf{P}]+\nabla_{\mathbf{r}} \cdot\left[\frac{c}{4 \pi} \mathbf{E} \times(\mathbf{B}-4 \pi \mathbf{M})\right] \\
& +\nabla_{\mathbf{r}} \cdot \sum_{\text {p.s. }} \int d U d J\left[\mathcal{B} f \mathbf{V}_{\mathbf{R}}\left\langle\frac{m}{2} \mathfrak{v}^{2}\right\rangle\right]_{\mathbf{R}=\mathbf{r}} \\
& -\boldsymbol{\nabla}_{\mathbf{r}} \cdot\left[\hat{\mathbf{e}}_{i}\left(\frac{\partial \mathbf{B}}{\partial t} \cdot \mathcal{M}_{i}\right)\right] \\
& -\sum_{\text {p.s. }} \int d^{3} R d U d J \mathcal{B} f\langle(\delta(\mathbf{r}-\mathbf{R})-\delta(\mathbf{r}-\mathfrak{x})) \\
& \left.\times\left[e \mathbf{E}(\mathfrak{x}, t) \cdot\left[\mathbf{V}_{\mathbf{R}}+\frac{\mathfrak{D} \boldsymbol{\rho}}{\mathfrak{D} t}\right]+\frac{\widehat{\partial}_{(\mathfrak{x}, t)} \mathcal{T}_{\mathfrak{v}}}{\partial t}\right]\right\rangle=0,
\end{aligned}
$$

where the last term $\widehat{\partial}_{(x, t)} \mathcal{T}_{\mathfrak{v}} / \partial t$ was obtained according to (A22). Summation with respect to $i=1,2,3$ is implied and the vectors $\hat{\mathbf{e}}_{i}$ are Cartesian basis vectors. In Appendix D, the same expression for the conserved local energy will be derived by a different method based on the equations of motion for the gyrocentres.

With (E1) taken into account, the expression containing the difference of the delta functions can be transformed to read

$$
-\boldsymbol{\nabla}_{\mathbf{r}} \cdot \sum_{\text {p. s. }} \int d^{3} R d U d J \mathcal{B} f\left\langle\mathbf{d}(\mathbf{r} ; \mathbf{R}, \mathfrak{x})\left[e \mathbf{E}(\mathfrak{x}, t) \cdot\left[\mathbf{V}_{\mathbf{R}}+\frac{\mathfrak{D} \boldsymbol{\rho}}{\mathfrak{D} t}\right]+\frac{\widehat{\partial}_{(x, t)} \mathcal{T}_{\mathfrak{v}}}{\partial t}\right]\right\rangle .
$$

Integration of (97) with respect to $\mathbf{r}$ yields the following expression for the total energy $\mathcal{E}$ of the system:

$$
\mathcal{E}=\int\left\{\frac{1}{8 \pi}\left[\mathbf{E}^{2}+\mathbf{B}^{2}\right]+\mathbf{E} \cdot \mathbf{P}+\sum_{\text {p. s. }} \int d U d J\left[\mathcal{B} f\left\langle\frac{m}{2} \mathfrak{v}^{2}\right\rangle\right]_{\mathbf{R}=\mathbf{r}}\right\} d^{3} r .
$$




\subsubsection{Local momentum conservation}

By proceeding as in the derivation of the local energy conservation law, one obtains, as a consequence of a space shift $\boldsymbol{\epsilon}_{\text {trans. }}$, an expression corresponding to $(91)$ :

$$
\begin{aligned}
\delta \mathcal{L}_{\text {tot. }}(\mathbf{r}, t) & =\delta^{\mathrm{EL}} \mathcal{L}_{\text {tot. }}(\mathbf{r}, t)=\delta \mathcal{L}_{\mathrm{M}}(\mathbf{r}, t)=-\boldsymbol{\epsilon}_{\text {trans. }} \cdot \nabla \mathcal{L}_{\mathrm{M}}(\mathbf{r}, t) \\
& =-\boldsymbol{\epsilon}_{\text {trans. }} \cdot \boldsymbol{\nabla}\left[\frac{1}{8 \pi}\left[\mathbf{E}^{2}(\mathbf{r}, t)-\mathbf{B}^{2}(\mathbf{r}, t)\right]\right]
\end{aligned}
$$

The variations of $S, \Phi$ and $\mathbf{A}$ owing to a displacement $\boldsymbol{\epsilon}_{\text {trans. }}$ (and a simultaneous gauge transformation) are given by (C31) and (C22)-(C25):

$$
\begin{gathered}
\left(\delta_{\text {comb. }} S\right)_{\text {constr. }}+\boldsymbol{\epsilon}_{\text {trans. }}\left\langle\frac{\partial \boldsymbol{\rho}}{\partial \mathbf{R}} \cdot \mathfrak{p}\right\rangle=-\boldsymbol{\epsilon}_{\text {trans. }} \cdot\langle m \mathfrak{v}\rangle, \\
\delta_{\text {comb. }} \Phi=\boldsymbol{\epsilon}_{\text {trans. }} \cdot \mathbf{E} \quad, \quad \delta_{\text {comb. }} \mathbf{A}=\boldsymbol{\epsilon}_{\text {trans. }} \times \mathbf{B} .
\end{gathered}
$$

The equation corresponding to (94) is then

$$
\begin{aligned}
\delta \mathcal{L}_{\mathrm{M}}(\mathbf{r}, t)= & \delta \Phi \frac{1}{4 \pi} \boldsymbol{\nabla} \cdot \mathbf{E}+\delta \mathbf{A} \cdot\left[\frac{1}{4 \pi c} \frac{\partial \mathbf{E}}{\partial t}-\frac{1}{4 \pi} \boldsymbol{\nabla} \times \mathbf{B}\right] \\
& -\boldsymbol{\epsilon}_{\text {trans. }} \cdot \frac{1}{4 \pi} \boldsymbol{\nabla} \bullet(\mathbf{E E})+\boldsymbol{\epsilon}_{\text {trans. }} \cdot \frac{1}{4 \pi c} \frac{\partial}{\partial t}(\mathbf{E} \times \mathbf{B}) \\
& +\boldsymbol{\epsilon}_{\text {trans. }} \cdot \frac{1}{4 \pi} \boldsymbol{\nabla}\left(\mathbf{B}^{2}\right)-\boldsymbol{\epsilon}_{\text {trans. }} \cdot \frac{1}{4 \pi} \boldsymbol{\nabla} \bullet(\mathbf{B B}) .
\end{aligned}
$$

To obtain this expression, Maxwell's equations were used, and also the relations $\boldsymbol{\nabla} \cdot\left(\mathbf{E} \delta_{\text {comb. }} \Phi\right)=\boldsymbol{\epsilon}_{\text {trans. }} \cdot(\boldsymbol{\nabla} \bullet(\mathbf{E E}))$ and $\boldsymbol{\nabla} \cdot\left[\left(\delta_{\text {comb. }} \mathbf{A}\right) \times \mathbf{B}\right]=-\boldsymbol{\epsilon}_{\text {trans. }} \cdot \boldsymbol{\nabla} \mathbf{B}^{2}$ $+\boldsymbol{\epsilon}_{\text {trans. }} \cdot(\boldsymbol{\nabla} \bullet(\mathbf{B B}))$, where $\boldsymbol{\nabla} \bullet$ denotes the divergence of a dyadic. The terms $\sim \delta \Phi$ and $\delta \mathbf{A}$ compensate equal terms in $\delta_{\delta \Phi}^{\mathrm{EL}} \mathcal{L}_{\mathrm{K}}(\mathbf{r}, t)$ and $\delta_{\delta \mathbf{A}}^{\mathrm{EL}} \mathcal{L}_{\mathrm{K}}(\mathbf{r}, t)$, respectively.

By setting the right-hand side of (100) equal to that of (93), evaluated with (101)- (102), one obtains the local momentum conservation law at $(\mathbf{r}, t)$ :

$$
\begin{aligned}
& +\frac{\partial}{\partial t}\left[\frac{1}{4 \pi c}(\mathbf{E} \times \mathbf{B})\right]+\frac{\partial}{\partial t} \sum_{\text {p. s. }} \int d U d J[\mathcal{B} f\langle m \mathfrak{v}\rangle]_{\mathbf{R}=\mathbf{r}} \\
& +\frac{\partial}{\partial t}\left[\frac{1}{c} \mathbf{P} \times \mathbf{B}\right] \\
& +\boldsymbol{\nabla}_{\mathbf{r}}\left[\frac{1}{8 \pi}\left[\mathbf{E}^{2}+\mathbf{B}^{2}\right]\right]-\boldsymbol{\nabla}_{\mathbf{r}} \bullet\left[\frac{1}{4 \pi}[\mathbf{E E}+\mathbf{B B}]\right] \\
& +\boldsymbol{\nabla}_{\mathbf{r}} \bullet \sum_{\text {p.s. }} \int d U d J\left[\mathcal{B} f\left(\mathbf{V}_{\mathbf{R}}\langle m \mathfrak{v}\rangle\right)\right]_{\mathbf{R}=\mathbf{r}}-\boldsymbol{\nabla}_{\mathbf{r}} \bullet[\mathbf{P E}] \\
& +\boldsymbol{\nabla}_{\mathbf{r}} \bullet[\mathbf{B M}]-\boldsymbol{\nabla}_{\mathbf{r}}[\mathbf{B} \cdot \mathbf{M}]+\boldsymbol{\nabla}_{\mathbf{r}} \bullet\left[\hat{\mathbf{e}}_{i}\left(\frac{\partial \mathbf{B}}{\partial \mathbf{r}} \cdot \mathcal{M}_{i}\right)\right]
\end{aligned}
$$




$$
+\sum_{\text {p.s. }} \int d^{3} R d U d J \mathcal{B} f\left\{\left\langle(\delta(\mathbf{r}-\mathbf{R})-\delta(\mathbf{r}-\mathfrak{x}))\left[\mathbf{G}-m \frac{\mathfrak{D} \mathfrak{v}}{\mathfrak{D} t}\right]\right\rangle\right\}=0
$$

where the last term was obtained by making use of (A23) for $\widehat{\partial}_{(\mathfrak{x}, t)} \mathcal{T}_{\mathfrak{v}} / \partial \mathfrak{x}$ and (A14) for G. Summation with respect to $i=1,2,3$ is implied and the $\hat{\mathbf{e}}_{i}$ are Cartesian basis vectors. The terms which are gradients could be written as divergences, e.g $\boldsymbol{\nabla}_{\mathbf{r}}[\mathbf{B} \cdot \mathbf{M}]=\boldsymbol{\nabla}_{\mathbf{r}} \bullet[(\mathbf{B} \cdot \mathbf{M}) \underline{\underline{\mathbf{I}}}]$, where $\underline{\underline{\mathbf{I}}}$ is the unity tensor. As in the case of the energy conservation law, the expression containing the difference of the delta functions can be transformed, with (E1) taken into account, to read

$$
\boldsymbol{\nabla}_{\mathbf{r}} \bullet \sum_{\text {p.s. }} \int d^{3} R d U d J \mathcal{B} f\left\{\left\langle\mathbf{d}(\mathbf{r} ; \mathbf{R}, \mathfrak{x})\left[\mathbf{G}-m \frac{\mathfrak{D} \mathfrak{v}}{\mathfrak{D} t}\right]\right\rangle\right\} .
$$

Integration of (104) with respect to $\mathbf{r}$ yields the following expression for the total momentum $\mathcal{P}$ of the system:

$$
\mathcal{P}=\int\left\{\frac{1}{4 \pi c}(\mathbf{E} \times \mathbf{B})+\frac{1}{c}(\mathbf{P} \times \mathbf{B})+\sum_{\text {p.s. }} \int d U d J[\mathcal{B} f\langle m \mathfrak{v}\rangle]_{\mathbf{R}=\mathbf{r}}\right\} d^{3} r .
$$

After the following derivation of the local angular momentum conservation law, we obtain an alternative expression for the local momentum conservation law in which all the tensors appearing in the spatial part are obviously symmetric.

\subsubsection{Local angular momentum conservation}

As usual, the local angular momentum conservation law is obtained from infinitesimal rotational invariance. We consider infinitesimal rotations $\boldsymbol{\zeta}_{\mathbf{R}}=$ $\boldsymbol{\epsilon}_{\text {rot. }} \times \mathbf{R}$ or, with the argument $\mathbf{r}, \boldsymbol{\zeta}_{\mathbf{r}}=\boldsymbol{\epsilon}_{\text {rot. }} \times \mathbf{r}$, as defined by (C1) with vanishing parallel shifts $\boldsymbol{\epsilon}_{\text {trans. }}=0$. As in the derivation of the local energy and momentum conservation laws, one obtains an expression for $\delta \mathcal{L}_{\text {tot. }}(\mathbf{r}, t)$ corresponding to (91) and (100), namely

$$
\begin{aligned}
\delta \mathcal{L}_{\text {tot. }}(\mathbf{r}, t) & =\delta^{\mathrm{EL}} \mathcal{L}_{\text {tot. }}(\mathbf{r}, t)=\delta \mathcal{L}_{\mathrm{M}}(\mathbf{r}, t)=-\boldsymbol{\zeta}_{\mathbf{r}} \cdot \nabla \mathcal{L}_{\mathrm{M}}(\mathbf{r}, t) \\
& =-\left(\boldsymbol{\epsilon}_{\text {rot. }} \times \mathbf{r}\right) \cdot \boldsymbol{\nabla} \mathcal{L}_{\mathrm{M}}(\mathbf{r}, t) \\
& =\boldsymbol{\epsilon}_{\text {rot. }} \cdot \boldsymbol{\nabla} \times\left[\frac{1}{8 \pi}\left[\mathbf{E}^{2}(\mathbf{r}, t)-\mathbf{B}^{2}(\mathbf{r}, t)\right] \mathbf{r}\right]
\end{aligned}
$$

The variations of $S, \Phi$ and $\mathbf{A}$ owing to an infinitesimal rotation (and a simultaneous gauge transformation) are given by (C31) and (C22)-(C25),

$$
\begin{gathered}
\left(\delta_{\text {comb. }} S\right)_{\text {constr. }}-\left\langle\left(\delta_{\delta \mathbf{A}} \boldsymbol{\rho}\right) \cdot \mathfrak{p}\right\rangle=-\boldsymbol{\epsilon}_{\text {rot. }} \cdot\langle\mathfrak{x} \times m \mathfrak{v}\rangle \\
\delta_{\text {comb. }} \Phi(\mathfrak{x}, t)=\boldsymbol{\epsilon}_{\text {rot. }} \cdot(\mathfrak{x} \times \mathbf{E}(\mathfrak{x}, t)) \quad, \quad \delta_{\text {comb. }} \Phi(\mathbf{r}, t)=\boldsymbol{\epsilon}_{\text {rot. }} \cdot(\mathbf{r} \times \mathbf{E}(\mathbf{r}, t)),
\end{gathered}
$$




$$
\delta_{\text {comb. }} \mathbf{A}(\mathfrak{x}, t)=\left(\boldsymbol{\epsilon}_{\text {rot. }} \times \mathfrak{x}\right) \times \mathbf{B}(\mathfrak{x}, t) \quad, \quad \delta_{\text {comb. }} \mathbf{A}(\mathbf{r}, t)=\left(\boldsymbol{\epsilon}_{\text {rot. }} \times \mathbf{r}\right) \times \mathbf{B}(\mathbf{r}, t)
$$

The equation corresponding to (94) is

$$
\begin{aligned}
\delta \mathcal{L}_{\mathrm{M}}(\mathbf{r}, t)= & \delta \Phi \frac{1}{4 \pi} \boldsymbol{\nabla} \cdot \mathbf{E}+\delta \mathbf{A} \cdot\left[\frac{1}{4 \pi c} \frac{\partial \mathbf{E}}{\partial t}-\frac{1}{4 \pi} \boldsymbol{\nabla} \times \mathbf{B}\right] \\
& -\boldsymbol{\epsilon}_{\text {rot. }} \cdot \frac{1}{4 \pi} \boldsymbol{\nabla} \bullet[\mathbf{E}(\mathbf{r} \times \mathbf{E})]+\boldsymbol{\epsilon}_{\text {rot. }} \cdot \frac{1}{4 \pi c} \frac{\partial}{\partial t}[\mathbf{r} \times(\mathbf{E} \times \mathbf{B})] \\
& -\boldsymbol{\epsilon}_{\text {rot. }} \cdot \frac{1}{4 \pi} \boldsymbol{\nabla} \times\left(\mathbf{B}^{2} \mathbf{r}\right)-\boldsymbol{\epsilon}_{\text {rot. }} \cdot \frac{1}{4 \pi} \boldsymbol{\nabla} \bullet[\mathbf{B}(\mathbf{r} \times \mathbf{B})],
\end{aligned}
$$

where $\mathbf{E}(\mathbf{r} \times \mathbf{E})$ and $\mathbf{B}(\mathbf{r} \times \mathbf{B})$ are dyadics built by the vectors $\mathbf{E}$ and $\mathbf{r} \times \mathbf{E}$ and $\mathbf{B}$ and $\mathbf{r} \times \mathbf{B}$, respectively. Use was made of Maxwell's equations and of the relations $\boldsymbol{\nabla} \cdot\left(\mathbf{E} \delta_{\text {comb. }} \Phi\right)=\boldsymbol{\epsilon}_{\text {rot. }} \cdot(\boldsymbol{\nabla} \bullet[\mathbf{E}(\mathbf{r} \times \mathbf{E})])$ and $\boldsymbol{\nabla} \cdot\left[\left(\delta_{\text {comb. }} \mathbf{A}\right) \times \mathbf{B}\right]=$ $\boldsymbol{\epsilon}_{\text {rot. }} \cdot \boldsymbol{\nabla} \times\left(\mathbf{B}^{2} \mathbf{r}\right)+\boldsymbol{\epsilon}_{\text {rot. }} \cdot(\boldsymbol{\nabla} \bullet[\mathbf{B}(\mathbf{r} \times \mathbf{B})])$. The terms $\sim \delta \Phi$ and $\delta \mathbf{A}$ compensate equal terms in $\delta_{\delta \Phi}^{\mathrm{EL}} \mathcal{L}_{\mathrm{K}}(\mathbf{r}, t)$ and $\delta_{\delta \mathbf{A}}^{\mathrm{EL}} \mathcal{L}_{\mathrm{K}}(\mathbf{r}, t)$, respectively.

By setting the right-hand side of (107) equal to that of (93), evaluated with (108)- (111), one obtains the local angular momentum conservation law at $(\mathbf{r}, t)$ :

$$
\begin{aligned}
& \left.\frac{\partial}{\partial t}\right|_{\mathbf{r}}\left[\frac{1}{4 \pi c} \mathbf{r} \times(\mathbf{E} \times \mathbf{B})\right]+\left.\frac{\partial}{\partial t}\right|_{\mathbf{r} \text {. s. }} \int d U d J[\mathcal{B} f\langle\mathfrak{x} \times m \mathfrak{v}\rangle]_{\mathbf{R}=\mathbf{r}} \\
& +\left.\frac{\partial}{\partial t}\right|_{\mathbf{r}}\left[\mathbf{r} \times\left[\frac{1}{c} \mathbf{P} \times \mathbf{B}\right]\right] \\
& -\nabla_{\mathbf{r}} \times\left[\frac{1}{8 \pi}\left[\mathbf{E}^{2}+\mathbf{B}^{2}\right] \mathbf{r}\right]-\nabla_{\mathbf{r}} \bullet\left[\frac{1}{4 \pi}[\mathbf{E}(\mathbf{r} \times \mathbf{E})+\mathbf{B}(\mathbf{r} \times \mathbf{B})]\right] \\
& +\nabla_{\mathbf{r}} \bullet \sum_{\mathrm{p.s.}} \int d U d J\left[\mathcal{B} f\left(\mathbf{V}_{\mathbf{R}}\langle\mathfrak{x} \times m \mathfrak{v}\rangle\right)\right]_{\mathbf{R}=\mathbf{r}}-\nabla_{\mathbf{r}} \bullet[\mathbf{P}(\mathbf{r} \times \mathbf{E})] \\
& +\nabla_{\mathbf{r}} \bullet[\mathbf{B}(\mathbf{r} \times \mathbf{M})]+\nabla_{\mathbf{r}} \times[(\mathbf{B} \cdot \mathbf{M}) \mathbf{r}]+\frac{\partial}{\partial r_{i}}\left[\mathbf{r} \times\left[\frac{\partial \mathbf{B}}{\partial \mathbf{r}} \cdot \mathcal{M}_{i}\right]+\mathcal{M}_{i} \times \mathbf{B}\right] \\
& +\sum_{\mathrm{p} . \mathbf{s .}} \int d^{3} R d U d J \mathcal{B} f\left\{\left\langle( \delta ( \mathbf { r } - \mathbf { R } ) - \delta ( \mathbf { r } - \mathfrak { x } ) ) \left[\mathfrak{x} \times\left(\mathbf{G}-m \frac{\mathfrak{D} \mathfrak{v}}{\mathfrak{D} t}\right)\right.\right.\right. \\
& \left.\left.\left.+m \mathfrak{v} \times \frac{\mathfrak{D} \mathfrak{x}}{\mathfrak{D} t}\right]\right\rangle\right\}=0,
\end{aligned}
$$

where the last terms multiplying the delta functions were obtained by making use of (A14), (A23) and (E8). Summation with respect to $i=1,2,3$ is, again, implied.

As in the case of the energy and momentum conservation laws, the expression containing the difference of the delta functions can be transformed, with (E1) taken into account, to read

$$
\boldsymbol{\nabla}_{\mathbf{r}} \bullet \sum_{\text {p. s. }} \int d^{3} R d U d J \mathcal{B} f\left\{\left\langle\mathbf { d } ( \mathbf { r } ; \mathbf { R } , \mathfrak { x } ) \left[\mathfrak{x} \times\left(\mathbf{G}-m \frac{\mathfrak{D} \mathfrak{v}}{\mathfrak{D} t}\right)\right.\right.\right.
$$




$$
\left.\left.\left.+m \mathfrak{v} \times \frac{\mathfrak{D} \mathfrak{x}}{\mathfrak{D} t}\right]\right\rangle\right\}
$$

Integration of (112) with respect to $\mathbf{r}$ yields the following expression for the total angular momentum $\mathbf{L}$ of the system:

$$
\begin{aligned}
\mathbf{L}= & \int\left\{\mathbf{r} \times\left[\frac{1}{4 \pi c}(\mathbf{E} \times \mathbf{B})+\frac{1}{c}(\mathbf{P} \times \mathbf{B})+\sum_{\text {p.s. }} \int d U d J[\mathcal{B} f\langle m \mathfrak{v}\rangle]_{\mathbf{R}=\mathbf{r}}\right]\right. \\
& \left.+\sum_{\text {p. s. }} \int d U d J[\mathcal{B} f\langle\boldsymbol{\rho} \times m \mathfrak{v}\rangle]_{\mathbf{R}=\mathbf{r}}\right\} d^{3} r
\end{aligned}
$$

where the relation $\delta(\mathbf{r}-\mathbf{R})\langle\mathfrak{x} \times m \mathfrak{v}\rangle=\delta(\mathbf{r}-\mathbf{R})[\mathbf{r} \times\langle m \mathfrak{v}\rangle+\langle\boldsymbol{\rho} \times m \mathfrak{v}\rangle]$ was used.

\subsubsection{Alternative form for the local momentum conservation law}

In the following, the local momentum conservation law is expressed in a form in which all the tensors appearing in the spatial part are obviously symmetric. This expression, together with the local energy conservation law, then immediately allows one to obtain the symmetric (with respect to the spatial components) energy-momentum tensor.

By building the vector product of the momentum conservation law, (104), with $\mathbf{r}$ and substracting the result from the angular momentum conservation law, (112), one obtains in a few steps,

$$
\begin{aligned}
& \sum_{\text {p. s. }} \int d U d J\left[\mathcal{B} f \mathbf{V}_{\mathbf{R}} \times\langle m \mathfrak{v}\rangle\right]_{\mathbf{R}=\mathbf{r}}-\mathbf{P} \times \mathbf{E}+\mathbf{B} \times \mathbf{M} \\
+ & \hat{\mathbf{e}}_{i} \times\left[\left(\frac{\partial \mathbf{B}}{\partial \mathbf{r}} \cdot \mathcal{M}_{i}\right)\right] \\
& +\sum_{\text {p.s. }} \int d^{3} R d U d J \mathcal{B} f\left\langle\mathbf{d}(\mathbf{r} ; \mathbf{R}, \mathfrak{x}) \times\left[\mathbf{G}-m \frac{\mathfrak{D} \mathfrak{v}}{\mathfrak{D} t}\right]\right\rangle \\
= & -\left.\frac{\partial}{\partial t}\right|_{\mathbf{r}} \sum_{\text {p. s. }} \int d U d J[\mathcal{B} f\langle(\mathfrak{x}-\mathbf{r}) \times m \mathfrak{v}\rangle]_{\mathbf{R}=\mathbf{r}} \\
& -\nabla_{\mathbf{r}} \bullet \sum_{\text {p. s. }} \int d U d J\left[\mathcal{B} f\left(\mathbf{V}_{\mathbf{R}}\langle(\mathfrak{x}-\mathbf{r}) \times m \mathfrak{v}\rangle\right)\right]_{\mathbf{R}=\mathbf{r}} \\
& -\nabla_{\mathbf{r}} \bullet\left[\hat{\mathbf{e}}_{i}\left(\mathcal{M}{ }_{i} \times \mathbf{B}\right)\right] \\
& -\nabla_{\mathbf{r}} \bullet \sum_{\text {p. s. }} \int d^{3} R d U d J \mathcal{B} f\left\{\left\langle\mathbf { d } ( \mathbf { r } ; \mathbf { R } , \mathfrak { x } ) \left[(\mathfrak{x}-\mathbf{r}) \times\left(\mathbf{G}-m \frac{\mathfrak{D} \mathfrak{v}}{\mathfrak{D} t}\right)\right.\right.\right. \\
& \left.\left.\left.+m \mathfrak{v} \times \frac{\mathfrak{D} \mathfrak{x}}{\mathfrak{D} t}\right]\right\rangle\right\} .
\end{aligned}
$$

The validity of this expression can be confirmed in a complicated way by direct calculation using the definitions of $\mathbf{P}, \mathbf{M}, \boldsymbol{M}_{i}$ and several expressions from Appendix A. The curl of (115), with the generally valid relations

$$
\boldsymbol{\nabla} \times[\mathbf{c} \times \mathbf{f}]=-\boldsymbol{\nabla} \bullet[\mathbf{c f}-\mathbf{f c}]
$$


and

$$
\begin{aligned}
\boldsymbol{\nabla} \times[\boldsymbol{\nabla} \bullet(\mathbf{g h})] & =\frac{\partial}{\partial r_{i}}\left[\boldsymbol{\nabla} \times g_{i} \mathbf{h}\right]=\boldsymbol{\nabla} \bullet\left[\hat{\mathbf{e}}_{i}\left(\boldsymbol{\nabla} \times\left(g_{i} \mathbf{h}\right)\right)\right] \\
& =\boldsymbol{\nabla} \bullet\left[\hat{\mathbf{e}}_{i}\left(\boldsymbol{\nabla} \times\left(g_{i} \mathbf{h}\right)\right)+\left(\boldsymbol{\nabla} \times\left(g_{i} \mathbf{h}\right)\right) \hat{\mathbf{e}}_{i}\right]
\end{aligned}
$$

for arbitrary vectors $\mathbf{c}, \mathbf{f}, \mathbf{g}, \mathbf{h}$ taken into account, yields

$$
\begin{aligned}
& \boldsymbol{\nabla}_{\mathbf{r}} \bullet \sum_{\text {p. s. }} \int d U d J\left[\mathcal{B} f\left(\mathbf{V}_{\mathbf{R}}\langle m \mathfrak{v}\rangle-\langle m \mathfrak{v}\rangle \mathbf{V}_{\mathbf{R}}\right)\right]_{\mathbf{R}=\mathbf{r}}-\nabla_{\mathbf{r}} \bullet[\mathbf{P E}-\mathbf{E P}] \\
& +\nabla_{\mathbf{r}} \bullet[\mathbf{B M}-\mathbf{M B}]+\nabla_{\mathbf{r}} \bullet\left[\hat{\mathbf{e}}_{i}\left(\frac{\partial \mathbf{B}}{\partial \mathbf{r}} \cdot \mathcal{M}_{i}\right)-\left(\frac{\partial \mathbf{B}}{\partial \mathbf{r}} \cdot \mathcal{M}_{i}\right) \hat{\mathbf{e}}_{i}\right] \\
& +\nabla_{\mathbf{r}} \bullet \sum_{\text {p.s. }} \int d^{3} R d U d J \mathcal{B} f\left\{\left\langle\mathbf{d}(\mathbf{r} ; \mathbf{R}, \mathfrak{x})\left(\mathbf{G}-m \frac{\mathfrak{D} \mathfrak{v}}{\mathfrak{D} t}\right)\right.\right. \\
& \left.\left.-\left(\mathbf{G}-m \frac{\mathfrak{D} \mathfrak{v}}{\mathfrak{D} t}\right) \mathbf{d}(\mathbf{r} ; \mathbf{R}, \mathfrak{x})\right\rangle\right\} \\
& =\left.\frac{\partial}{\partial t}\right|_{\mathbf{r}} \boldsymbol{\nabla}_{\mathbf{r}} \times\left[\sum_{\text {p.s. }} \int d U d J[\mathcal{B} f\langle(\mathfrak{x}-\mathbf{r}) \times m \mathfrak{v}\rangle]_{\mathbf{R}=\mathbf{r}}\right] \\
& +\nabla_{\mathbf{r}} \times\left[\nabla_{\mathbf{r}} \bullet \sum_{\text {p.s. }} \int d U d J\left[\mathcal{B} f\left(\mathbf{V}_{\mathbf{R}}\langle(\mathfrak{x}-\mathbf{r}) \times m \mathfrak{v}\rangle\right)\right]_{\mathbf{R}=\mathbf{r}}\right] \\
& +\nabla_{\mathbf{r}} \times\left[\nabla_{\mathbf{r}} \bullet\left[\hat{\mathbf{e}}_{i}\left(\mathcal{M}_{i} \times \mathbf{B}\right)\right]\right] \\
& +\nabla_{\mathbf{r}} \times\left[\nabla _ { \mathbf { r } } \bullet \sum _ { \text { p. s. } } \int d ^ { 3 } R d U d J \mathcal { B } f \left\{\left\langle\mathbf { d } ( \mathbf { r } ; \mathbf { R } , \mathfrak { x } ) \left[(\mathfrak{x}-\mathbf{r}) \times\left(\mathbf{G}-m \frac{\mathfrak{D} \mathfrak{v}}{\mathfrak{D} t}\right)\right.\right.\right.\right. \\
& \left.\left.\left.\left.+m \mathfrak{v} \times \frac{\mathfrak{D} \mathfrak{x}}{\mathfrak{D} t}\right]\right\rangle\right\}\right] \\
& =\left.\frac{\partial}{\partial t}\right|_{\mathbf{r}} \boldsymbol{\nabla}_{\mathbf{r}} \times\left[\sum_{\text {p.s. }} \int d U d J[\mathcal{B} f\langle(\mathfrak{x}-\mathbf{r}) \times m \mathfrak{v}\rangle]_{\mathbf{R}=\mathbf{r}}\right] \\
& +\nabla_{\mathbf{r}} \bullet\left[\hat{\mathbf{e}}_{i}\left(\nabla_{\mathbf{r}} \times \sum_{\text {p.s. }} \int d U d J\left[\mathcal{B} f\left(\mathbf{V}_{\mathbf{R}} \cdot \hat{\mathbf{e}}_{i}\right)\langle(\mathfrak{x}-\mathbf{r}) \times m \mathfrak{v}\rangle\right]_{\mathbf{R}=\mathbf{r}}\right)\right. \\
& \left.+\left(\nabla_{\mathbf{r}} \times \sum_{\text {p.s. }} \int d U d J\left[\mathcal{B} f\left(\mathbf{V}_{\mathbf{R}} \cdot \hat{\mathbf{e}}_{i}\right)\langle(\mathfrak{x}-\mathbf{r}) \times m \mathfrak{v}\rangle\right]_{\mathbf{R}=\mathbf{r}}\right) \hat{\mathbf{e}}_{i}\right] \\
& +\nabla_{\mathbf{r}} \bullet\left[\hat{\mathbf{e}}_{i}\left(\nabla_{\mathbf{r}} \times\left[\mathcal{M}_{i} \times \mathbf{B}\right]\right)+\left(\nabla_{\mathbf{r}} \times\left[\mathcal{M}_{i} \times \mathbf{B}\right]\right) \hat{\mathbf{e}}_{i}\right] \\
& +\nabla_{\mathbf{r}} \bullet\left[\hat { \mathbf { e } } _ { i } \left(\nabla_{\mathbf{r}} \times \sum_{\text {p.s. }} \int d^{3} R d U d J \mathcal{B} f\left\{\left\langle( \mathbf { d } ( \mathbf { r } ; \mathbf { R } , \mathfrak { x } ) \cdot \hat { \mathbf { e } } _ { i } ) \left[(\mathfrak{x}-\mathbf{r}) \times\left(\mathbf{G}-m \frac{\mathfrak{D} \mathfrak{v}}{\mathfrak{D} t}\right)\right.\right.\right.\right.\right. \\
& \left.\left.\left.\left.+m \mathfrak{v} \times \frac{\mathfrak{D} \mathfrak{x}}{\mathfrak{D} t}\right]\right\rangle\right\}\right) \\
& +\left(\boldsymbol{\nabla}_{\mathbf{r}} \times \sum_{\text {p.s. }} \int d^{3} R d U d J \mathcal{B} f\left\{\left\langle( \mathbf { d } ( \mathbf { r } ; \mathbf { R } , \mathfrak { x } ) \cdot \hat { \mathbf { e } } _ { i } ) \left[(\mathfrak{x}-\mathbf{r}) \times\left(\mathbf{G}-m \frac{\mathfrak{D} \mathfrak{v}}{\mathfrak{D} t}\right)\right.\right.\right.\right. \\
& \left.\left.\left.\left.\left.+m \mathfrak{v} \times \frac{\mathfrak{D} \mathfrak{x}}{\mathfrak{D} t}\right]\right\rangle\right\}\right) \hat{\mathbf{e}}_{i}\right] .
\end{aligned}
$$


By introducing the definitions

$$
\begin{gathered}
\mathbf{s}:=\boldsymbol{\rho} \times m \mathbf{v} \\
\mathbf{W}_{i \mathbf{s}}(\mathbf{r}, t):=\nabla_{\mathbf{r}} \times \sum_{\text {p.s. }} \int d U d J\left[\mathcal{B} f\left(\mathbf{V}_{\mathbf{R}} \cdot \hat{\mathbf{e}}_{i}\right)\langle\mathbf{s}\rangle\right]_{\mathbf{R}=\mathbf{r}}, \\
\mathbf{W}_{\mathcal{M}_{i}(\mathbf{r}, t)}:=\nabla_{\mathbf{r}} \times\left[\mathcal{M}_{i} \times \mathbf{B}\right] \\
\mathbf{W}_{i \mathbf{d}}(\mathbf{r}, t):=\nabla_{\mathbf{r}} \times \sum_{\text {p. s. }} \int d^{3} R d U d J \mathcal{B} f\left\{\left\langle( \mathbf { d } ( \mathbf { r } ; \mathbf { R } , \mathfrak { x } ) \cdot \hat { \mathbf { e } } _ { i } ) \left[(\mathfrak{x}-\mathbf{r}) \times\left(\mathbf{G}-m \frac{\mathfrak{D} \mathfrak{v}}{\mathfrak{D} t}\right)\right.\right.\right. \\
\left.\left.\left.+m \mathfrak{v} \times \frac{\mathfrak{D} \mathfrak{x}}{\mathfrak{D} t}\right]\right\rangle\right\} \\
\mathbf{W}_{i}=\mathbf{W}_{i \mathbf{s}}+\mathbf{W}_{\mathcal{M}_{i}}+\mathbf{W}_{i \mathbf{d}},
\end{gathered}
$$

the right-hand side of (118) can be written as

$$
\begin{aligned}
& \left.\frac{\partial}{\partial t}\right|_{\mathbf{r}}\left[\nabla_{\mathbf{r}} \times \sum_{\text {p.s. }} \int d U d J[\mathcal{B} f\langle\mathbf{s}\rangle]_{\mathbf{R}=\mathbf{r}}\right] \\
& +\boldsymbol{\nabla}_{\mathbf{r}} \bullet\left[\hat{\mathbf{e}}_{i} \mathbf{W}_{i}+\mathbf{W}_{i} \hat{\mathbf{e}}_{i}\right] .
\end{aligned}
$$

This result allows one to express the spatial part of the local momentum conservation law, (104), in terms of symmetric tensors only. By writing $\mathbf{P E}=$ $[\mathbf{P E}+\mathbf{E P}] / 2+[\mathbf{P E}-\mathbf{E P}] / 2$, and similarly for other terms, one then obtains

$$
\begin{aligned}
& +\frac{\partial}{\partial t}\left[\frac{1}{4 \pi c}(\mathbf{E} \times \mathbf{B})\right]+\frac{\partial}{\partial t} \sum_{\text {p.s. }} \int d U d J[\mathcal{B} f\langle m \mathfrak{v}\rangle]_{\mathbf{R}=\mathbf{r}} \\
& +\frac{\partial}{\partial t}\left[\frac{1}{c} \mathbf{P} \times \mathbf{B}\right]+\frac{\partial}{\partial t}\left[\nabla_{\mathbf{r}} \times \frac{1}{2} \sum_{\text {p.s. }} \int d U d J[\mathcal{B} f\langle\mathbf{s}\rangle]_{\mathbf{R}=\mathbf{r}}\right] \\
& +\boldsymbol{\nabla}_{\mathbf{r}}\left[\frac{1}{8 \pi}\left[\mathbf{E}^{2}+\mathbf{B}^{2}\right]\right]-\nabla_{\mathbf{r}} \bullet\left[\frac{1}{4 \pi}[\mathbf{E E}+\mathbf{B B}]\right] \\
& +\nabla_{\mathbf{r}} \bullet \sum_{\text {p.s. }} \int d U d J\left[\mathcal{B} f \frac{1}{2}\left(\mathbf{V}_{\mathbf{R}}\langle m \mathfrak{v}\rangle+\langle m \mathfrak{v}\rangle \mathbf{V}_{\mathbf{R}}\right)\right]_{\mathbf{R}=\mathbf{r}} \\
& -\nabla_{\mathbf{r}} \bullet \frac{1}{2}[\mathbf{P E}+\mathbf{E P}]+\nabla_{\mathbf{r}} \bullet \frac{1}{2}[\mathbf{B M}+\mathbf{M B}] \\
& -\nabla_{\mathbf{r}}[\mathbf{B} \cdot \mathbf{M}]+\nabla_{\mathbf{r}} \bullet \frac{1}{2}\left[\hat{\mathbf{e}}_{i}\left(\frac{\partial \mathbf{B}}{\partial \mathbf{r}} \cdot \mathcal{M}_{i}\right)+\left(\frac{\partial \mathbf{B}}{\partial \mathbf{r}} \cdot \mathcal{M}_{i}\right) \hat{\mathbf{e}}_{i}\right] \\
& +\nabla_{\mathbf{r}} \bullet \sum_{\text {p. s. }} \int d^{3} R d U d J \mathcal{B} f\left\{\frac { 1 } { 2 } \left\langle\mathbf{d}(\mathbf{r} ; \mathbf{R}, \mathfrak{x})\left[\mathbf{G}-m \frac{\mathfrak{D} \mathfrak{v}}{\mathfrak{D} t}\right]\right.\right. \\
& \left.\left.+\left[\mathbf{G}-m \frac{\mathfrak{D} \mathfrak{v}}{\mathfrak{D} t}\right] \mathbf{d}(\mathbf{r} ; \mathbf{R}, \mathfrak{x})\right\rangle\right\} \\
& +\nabla_{\mathbf{r}} \bullet \frac{1}{2}\left[\hat{\mathbf{e}}_{i} \mathbf{W}_{i}+\mathbf{W}_{i} \hat{\mathbf{e}}_{i}\right]=0 .
\end{aligned}
$$

Again, summation with respect to $i=1,2,3$ is implied. 


\subsubsection{Energy-momentum tensor}

For describing the energy-momentum tensor it is convenient to introduce a four-vector representation with

$$
\mathbf{r}^{0}=c t \quad, \quad \mathbf{r}=\left(r^{1}, r^{2}, r^{3}\right) .
$$

The metric tensor $g^{\mu \nu}$ for this system is diagonal, with elements $g_{00}=-1$, $g_{11}=g_{22}=g_{33}=1$, which means that the 0 -components change their sign when the index 0 is shifted up and down.

The components of the energy-momentum tensor $\underline{\underline{T}}$ can be read from the expressions for the energy and momentum conservation, (97) and (125), respectively. From the energy conservation law one obtains

$$
\begin{aligned}
T^{00}= & \frac{1}{8 \pi}\left[\mathbf{E}^{2}+\mathbf{B}^{2}\right]+\mathbf{E} \cdot \mathbf{P}+\sum_{\text {p. s. }} \int d U d J\left[\mathcal{B} f\left\langle\frac{m}{2} \mathfrak{v}^{2}\right\rangle\right]_{\mathbf{R}=\mathbf{r}}, \\
T^{k 0}= & {\left[\frac{1}{4 \pi} \mathbf{E} \times(\mathbf{B}-4 \pi \mathbf{M})-\sum_{i=1}^{i=3} \hat{\mathbf{e}}_{i}\left(\frac{1}{c} \frac{\partial \mathbf{B}}{\partial t} \cdot \mathcal{M}_{i}\right)\right.} \\
& +\frac{1}{c} \sum_{\text {p. s. }} \int d U d J\left[\mathcal{B} f \mathbf{V}_{\mathbf{R}}\left\langle\frac{m}{2} \mathfrak{v}^{2}\right\rangle\right]_{\mathbf{R}=\mathbf{r}} \\
& -\frac{1}{c} \sum_{\text {p.s. }} \int d^{3} R d U d J \mathcal{B} f\left\langle\mathbf{d}(\mathbf{r}, \mathbf{R}, \mathfrak{x})\left[e \mathbf{E}(\mathfrak{x}, t) \cdot \frac{\mathfrak{D} \mathfrak{x}}{\mathfrak{D} t}+\frac{\widehat{\partial}_{(\mathfrak{x}, t)} \mathcal{T}_{\mathfrak{v}}}{\partial t}\right]\right\rangle_{k} \\
& k=1,2,3 .
\end{aligned}
$$

The momentum conservation law yields the components $T^{0 k}$ and the obviously symmetric spatial part, $(\underline{\underline{\mathrm{T}}})_{\text {space }}$ of the energy-momentum tensor:

$$
\begin{aligned}
T^{0 k}= & {\left[\frac{1}{4 \pi}(\mathbf{E} \times \mathbf{B})+\mathbf{P} \times \mathbf{B}+c \sum_{\text {p.s. }} \int d U d J[\mathcal{B} f\langle m \mathfrak{v}\rangle]_{\mathbf{R}=\mathbf{r}}\right.} \\
& \left.+c \boldsymbol{\nabla}_{\mathbf{r}} \times \frac{1}{2} \sum_{\text {p.s. }} \int d U d J[\mathcal{B} f\langle\mathbf{s}\rangle]_{\mathbf{R}=\mathbf{r}}\right]_{k},
\end{aligned}
$$

$k=1,2,3$, and

$$
\begin{aligned}
(\underline{\underline{\mathbf{T}}})_{\text {space }}= & T^{i k} \hat{\mathbf{e}}_{i} \hat{\mathbf{e}}_{k} \\
= & \frac{1}{8 \pi}\left[\mathbf{E}^{2}+\mathbf{B}^{2}\right] \underline{\underline{\mathbf{I}}}-\frac{1}{4 \pi}[\mathbf{E E}+\mathbf{B B}]-\frac{1}{2}[\mathbf{P E}+\mathbf{E P}] \\
& +\frac{1}{2}[\mathbf{B M}+\mathbf{M B}]-(\mathbf{B} \cdot \mathbf{M}) \underline{\underline{\mathbf{I}}} \\
& +\frac{1}{2} \sum_{j=1}^{j=3}\left[\hat{\mathbf{e}}_{j}\left(\frac{\partial \mathbf{B}}{\partial \mathbf{r}} \cdot \mathcal{M}_{j}\right)+\left(\frac{\partial \mathbf{B}}{\partial \mathbf{r}} \cdot \mathcal{M}_{j}\right) \hat{\mathbf{e}}_{j}\right]
\end{aligned}
$$




$$
\begin{aligned}
& +\frac{1}{2} \sum_{\mathbf{p . s .}} \int d U d J\left[\mathcal{B} f\left(\mathbf{V}_{\mathbf{R}}\langle m \mathfrak{v}\rangle+\langle m \mathfrak{v}\rangle \mathbf{V}_{\mathbf{R}}\right)\right]_{\mathbf{R}=\mathbf{r}} \\
& +\frac{1}{2} \sum_{\text {p. s. }} \int d^{3} R d U d J \mathcal{B} f\left\{\left\langle\mathbf{d}(\mathbf{r}, \mathbf{R}, \mathfrak{x})\left[\mathbf{G}-m \frac{\mathfrak{D} \mathbf{v}}{\mathfrak{D} t}\right]\right.\right. \\
& \left.\left.+\left[\mathbf{G}-m \frac{\mathfrak{D} \mathbf{v}}{\mathfrak{D} t}\right] \mathbf{d}(\mathbf{r}, \mathbf{R}, \mathfrak{x})\right\rangle\right\} \\
& +\frac{1}{2} \sum_{j=1}^{j=3}\left[\hat{\mathbf{e}}_{j} \mathbf{W}_{j}+\mathbf{W}_{j} \hat{\mathbf{e}}_{j}\right]
\end{aligned}
$$

where $\underline{\underline{I}}$ is the unity tensor. It should again be remarked that the symmetry of the energy-momentum tensor with respect to the spatial components is the only symmetry required for nonrelativistic theories. Within the framework of drift-kinetic theory, the symmetric spatial part of the energy-momentum tensor can directly be obtained from the momentum conservation law in the form given by (104).

It is not very difficult to show, though at some length, that the conservation laws for energy, momentum and angular momentum, and the negative of the energy-momentum tensor obtained here reduce in the drift-kinetic case to the expressions found in [11].

\section{Summary}

This paper is a continuation of the work on local energy and momentumconserving Maxwell-collisionless kinetic theories begun in $[1,2]$. These theories are based on a fully gauge-invariant Lagrangian. As concerns the kinetic part of this Lagrangian, three specific methods are used: first, Kruskal's formal infinite-series formalism for finding gyrophase-averaging coordinates for the particle orbits [13]; second, Dirac's constraint theory for obtaining a Hamiltonian for the particle motion from the nonstandard phase-space particle Lagrangian in averaging coordinates $[15,16]$; and, third, the Hamilton-Jacobi equation as an intermediate tool providing a Eulerian description of the particle motion. Within this kind of theory the following results were obtained:

1. A clearly defined kinetic density, (78).

2. The kinetic equation for each particle species, (45).

3. The polarization $\mathbf{P},(62)$.

4. The magnetization $\mathbf{M}$, (68)-(71).

5. The charge density $\rho,(59)-(61)$.

6. The current density $\mathbf{j},(65)-(71)$. 
7. The local energy conservation law, (97).

8. The local momentum conservation law, (104) and (125).

9. The local angular momentum conservation law, (112).

10. Physically very clear expressions for the total energy, momentum and angular momentum, (99), (106), and (114), respectively

11. The symmetric energy-momentum tensor (symmetric with respect to the spatial components only, as required in nonrelativistic theories), (126)(129).

The conservation laws for energy, momentum and angular momentum became very clear by expressing them in terms of the polarization, the magnetization and a third, very transparent expression $\mathcal{T}_{\mathfrak{v}}$ defined in (A10). This transparency concerns also the occurrence of a non-local term in each of the three conservation laws.

\section{Appendix A. Symbols and useful definitions}

The following definitions are useful for describing variations involving $\delta \Phi$ and $\delta \mathbf{A}$.

Let $K\left(g_{i}(\mathbf{R}, U ; J ; t), h_{j}(\mathfrak{x}, t)\right)$ be any function depending on a set of functions $g_{i}(\mathbf{R}, U ; J ; t)$ and/or functions $h_{j}(\mathfrak{x}, t)$, e.g. $K=(m / 2) U^{2}+$ const.B(R, $\left.t\right)+$ $e \Phi(\mathfrak{x}, t)$. We then define the following quantities and operators referring to the arguments of the functions $g_{i}(\mathbf{R}, U ; J ; t)$ and $h_{j}(\mathfrak{x}, t)$ :

$$
\begin{aligned}
\frac{\partial_{(\mathbf{R}, U ; J ; t)} K}{\partial \mathbf{R}} & =\left.\sum_{i} \frac{\partial g_{i}}{\partial \mathbf{R}}\right|_{U, J, t} \frac{\partial K}{\partial g_{i}} \\
\frac{\partial_{(\mathbf{R}, U ; J ; t)} K}{\partial t} & =\left.\sum_{i} \frac{\partial g_{i}}{\partial t}\right|_{\mathbf{R}, U, J} \frac{\partial K}{\partial g_{i}} \\
\frac{\partial_{(x, t)} K}{\partial \mathfrak{x}} & =\left.\sum_{j} \frac{\partial h_{j}}{\partial \mathfrak{x}}\right|_{t} \frac{\partial K}{\partial h_{j}} \\
\frac{\partial_{(x, t)} K}{\partial t} & =\left.\sum_{j} \frac{\partial h_{j}}{\partial t}\right|_{\mathfrak{x}} \frac{\partial K}{\partial h_{j}}
\end{aligned}
$$

and, correspondingly, for the derivatives with respect to $U$ and $J$ in $g_{i}(\mathbf{R}, U ; J ; t)$. The time derivative at constant $R, U, J, \phi$ is therefore given by

$$
\left.\frac{\partial K}{\partial t}\right|_{\mathbf{R}, U, J, \phi}=\frac{\partial_{(\mathbf{R}, U ; J ; t)} K}{\partial t}+\frac{\partial_{(x, t)} K}{\partial t}+\frac{\partial \mathfrak{x}}{\partial t} \cdot \frac{\partial_{(\mathfrak{x}, t)} K}{\partial \mathfrak{x}}
$$


and, correspondingly,

$$
\begin{aligned}
& \left.\frac{\partial K}{\partial \mathbf{R}}\right|_{U, J, \phi, t}=\frac{\partial_{(\mathbf{R}, U ; J ; t)} K}{\partial \mathbf{R}}+\frac{\partial \mathfrak{x}}{\partial \mathbf{R}} \cdot \frac{\partial_{(\mathfrak{x}, t)} K}{\partial \mathfrak{x}} \\
& \left.\frac{\partial K}{\partial U}\right|_{\mathbf{R}, J, \phi, t}=\frac{\partial_{(\mathbf{R}, U ; J ; t)} K}{\partial U}+\frac{\partial \mathfrak{x}}{\partial U} \cdot \frac{\partial_{(x, t)} K}{\partial \mathfrak{x}} .
\end{aligned}
$$

The time derivative in the coordinates $\mathbf{R}, U, J, \phi ; t$, without the fast gyroangle dependence, is defined as

$$
\left.\frac{\mathfrak{D}}{\mathfrak{D} t} \equiv \frac{\partial}{\partial t}\right|_{\mathbf{R}, U, J, \phi}+\mathbf{V}_{\mathbf{R}} \cdot \frac{\partial}{\partial \mathbf{R}}+V_{U} \frac{\partial}{\partial U},
$$

and, therefore,

$$
\begin{aligned}
\frac{\mathfrak{D} K}{\mathfrak{D} t}= & \frac{\partial K}{\partial t}+\mathbf{V}_{\mathbf{R}} \cdot \frac{\partial K}{\partial \mathbf{R}}+V_{U} \frac{\partial K}{\partial U} \\
= & \frac{\partial_{(\mathfrak{x}, t)} K}{\partial t}+\frac{\partial \mathfrak{x}}{\partial t} \cdot \frac{\partial_{(\mathfrak{x}, t)} K}{\partial \mathfrak{x}}+\mathbf{V}_{\mathbf{R}} \cdot \frac{\partial \mathfrak{x}}{\partial \mathbf{R}} \cdot \frac{\partial_{(\mathfrak{x}, t)} K}{\partial \mathfrak{x}}+V_{U} \frac{\partial \mathfrak{x}}{\partial U} \cdot \frac{\partial_{(x, t)} K}{\partial \mathfrak{x}} \\
& +\frac{\partial_{(\mathbf{R}, U ; J ; t)} K}{\partial t}+\mathbf{V}_{\mathbf{R}} \cdot \frac{\partial_{(\mathbf{R}, U ; J ; t)} K}{\partial \mathbf{R}}+V_{U} \frac{\partial_{(\mathbf{R}, U ; J ; t)} K}{\partial U} \\
= & \frac{\partial_{(x, t)} K}{\partial t}+\frac{\mathfrak{D} \mathfrak{x}}{\mathfrak{D} t} \cdot \frac{\partial_{(\mathfrak{x}, t)} K}{\partial \mathfrak{x}} \\
& +\frac{\partial_{(\mathbf{R}, U ; J ; t)} K}{\partial t}+\mathbf{V}_{\mathbf{R}} \cdot \frac{\partial_{(\mathbf{R}, U ; J ; t)} K}{\partial \mathbf{R}}+V_{U} \frac{\partial_{(\mathbf{R}, U ; J ; t)}}{\partial U}
\end{aligned}
$$

We further define

$$
\mathcal{T}_{\mathfrak{v}} \equiv \frac{m}{2} \mathfrak{v}^{2}-m \mathfrak{v} \cdot \frac{\mathfrak{D} \mathfrak{x}}{\mathfrak{D} t}=\frac{m}{2} \mathfrak{v}^{2}-m \mathfrak{v} \cdot\left[\mathbf{V}_{\mathbf{R}}+\frac{\mathfrak{D} \boldsymbol{\rho}}{\mathfrak{D} t}\right]
$$

with

$$
\begin{gathered}
\boldsymbol{\rho} \equiv \mathfrak{x}(\mathbf{R}, U, J, \phi ; t)-\mathbf{R} \\
\widehat{\partial} \mathcal{T}_{\mathfrak{v}} \equiv \frac{m}{2} \partial \mathfrak{v}^{2}-m(\partial \mathfrak{v}) \cdot\left[\mathbf{V}_{\mathbf{R}}+\frac{\mathfrak{D} \boldsymbol{\rho}}{\mathfrak{D} t}\right] \\
\widehat{\partial}_{(\mathfrak{x}, t)} \mathcal{T}_{\mathfrak{v}} \equiv \frac{m}{2} \partial_{(\mathfrak{x}, t)} \mathfrak{v}^{2}-m\left(\partial_{(\mathfrak{x}, t)} \mathfrak{v}\right) \cdot\left[\mathbf{V}_{\mathbf{R}}+\frac{\mathfrak{D} \boldsymbol{\rho}}{\mathfrak{D} t}\right] \\
\mathbf{G} \equiv m \frac{\mathfrak{D} \mathfrak{v}}{\mathfrak{D} t}-e \mathbf{E}(\mathfrak{x}, t)-\frac{e}{c}\left[\mathbf{V}_{\mathbf{R}}+\frac{\mathfrak{D} \boldsymbol{\rho}}{\mathfrak{D} t}\right] \times \mathbf{B}(\mathfrak{x}, t)+\frac{\widehat{\partial}_{(\mathfrak{x}, t)} \mathcal{T}_{\mathfrak{v}}}{\partial \mathfrak{x}} \\
\mathcal{T}_{\boldsymbol{\rho}} \equiv \boldsymbol{\rho} \cdot \mathbf{G}, \widehat{\partial} \mathcal{T}_{\boldsymbol{\rho}}=(\partial \boldsymbol{\rho}) \cdot \mathbf{G} \\
\mathcal{T} \equiv \mathcal{T}_{\mathfrak{v}}+\mathcal{T}_{\boldsymbol{\rho}}
\end{gathered}
$$


Thus, in the case of a product, the differential operator $\widehat{\partial}$ acts only on the first term.

In all cases of interest here, $\boldsymbol{\rho}$ depends neither on the electric field nor on $\mathbf{B}(\mathfrak{x}, t)$. It depends on the fields only through $\mathbf{B}(\mathbf{R}, t)$ and $\mathbf{B}, R_{i}(\mathbf{R}, t)$. One can then simplify some quantities in $\delta_{\delta \Phi} \mathcal{L}_{\mathrm{K}}(\mathbf{r}, t)$ and $\delta_{\delta \mathbf{A}} \mathcal{L}_{\mathrm{K}}(\mathbf{r}, t)$ according to the following relations:

$$
\begin{aligned}
\frac{\widehat{\partial}_{(\mathbf{R}, U, J ; t)}\langle\mathcal{T}\rangle}{\partial \mathbf{E}(\mathbf{R}, t)} & =\frac{\widehat{\partial}_{(\mathbf{R}, U, J ; t)}\left\langle\mathcal{T}_{\mathfrak{v}}\right\rangle}{\partial \mathbf{E}(\mathbf{R}, t)} \\
\frac{\widehat{\partial}_{(\mathfrak{x}, t)} \mathcal{T}}{\partial \mathbf{E}(\mathfrak{x}, t)} & =\frac{\widehat{\partial}_{(\mathfrak{x}, t)} \mathcal{T}_{\mathfrak{v}}}{\partial \mathbf{E}(\mathfrak{x}, t)} \\
\frac{\widehat{\partial}_{(\mathfrak{x}, t)} \mathcal{T}}{\partial \mathbf{B}(\mathfrak{x}, t)} & =\frac{\widehat{\partial}_{(\mathfrak{x}, t)} \mathcal{T}_{\mathfrak{v}}}{\partial \mathbf{B}(\mathfrak{x}, t)} \\
\frac{\widehat{\partial}_{(\mathfrak{x}, t)} \mathcal{T}}{\partial \mathbf{B}_{\mathrm{x}_{\mathfrak{i}}}(\mathfrak{x}, t)} & =\frac{\widehat{\partial}_{(\mathfrak{x}, t)} \mathcal{T}_{\mathfrak{v}}}{\partial \mathbf{B}_{\mathrm{x}_{\mathfrak{i}}}(\mathfrak{x}, t)}
\end{aligned}
$$

Further useful relations are

$$
\begin{aligned}
& \left.\frac{\widehat{\partial} \mathcal{T}_{\mathfrak{v}}}{\partial t}\right|_{\mathbf{R}, U, J, \phi}=\frac{\widehat{\partial}_{(\mathbf{R}, U, J ; t)} \mathcal{T}_{\mathfrak{v}}}{\partial t}+\frac{\widehat{\partial}_{(\mathfrak{x}, t)} \mathcal{T}_{\mathfrak{v}}}{\partial t}+\frac{\partial \mathfrak{x}}{\partial t} \cdot \frac{\widehat{\partial}_{(\mathfrak{x}, t)} \mathcal{T}_{\mathfrak{v}}}{\partial \mathfrak{x}} \\
& \frac{\widehat{\partial}_{(x, t)} \mathcal{T}_{\mathfrak{v}}}{\partial t}=\frac{\partial \mathbf{E}(\mathfrak{x}, t)}{\partial t} \cdot \frac{\widehat{\partial}_{(\mathfrak{x}, t)} \mathcal{T}_{\mathfrak{v}}}{\partial \mathbf{E}(\mathfrak{x}, t)}+\frac{\partial \mathbf{B}(\mathfrak{x}, t)}{\partial t} \cdot \frac{\widehat{\partial}_{(x, t)} \mathcal{T}_{\mathfrak{v}}}{\partial \mathbf{B}(\mathfrak{x}, t)}+\frac{\partial \mathbf{B},,_{\mathrm{x}_{\mathrm{i}}}(\mathfrak{x}, t)}{\partial t} \cdot \frac{\widehat{\partial}_{(x, t)} \mathcal{T}_{\mathfrak{v}}}{\partial \mathbf{B}_{, \mathrm{x}_{\mathrm{i}}}(\mathfrak{x}, t)} \\
& \frac{\widehat{\partial}_{(\mathfrak{x}, t)} \mathcal{T}_{\mathfrak{v}}}{\partial \mathfrak{x}}=\frac{\partial \mathbf{E}(\mathfrak{x}, t)}{\partial \mathfrak{x}} \cdot \frac{\widehat{\partial}_{(\mathfrak{x}, t)} \mathcal{T}_{\mathfrak{v}}}{\partial \mathbf{E}(\mathfrak{x}, t)}+\frac{\partial \mathbf{B}(\mathfrak{x}, t)}{\partial \mathfrak{x}} \cdot \frac{\widehat{\partial}_{(\mathfrak{x}, t)} \mathcal{T}_{\mathfrak{v}}}{\partial \mathbf{B}(\mathfrak{x}, t)}+\frac{\partial \mathbf{B}_{, \mathrm{x}_{\mathrm{i}}}(\mathfrak{x}, t)}{\partial \mathfrak{x}} \cdot \frac{\widehat{\partial}_{(\mathfrak{x}, t)} \mathcal{T}_{\mathfrak{v}}}{\partial \mathbf{B}_{, \mathrm{x}_{\mathrm{i}}}(\mathfrak{x}, t)} \\
& \frac{\widehat{\partial}_{(\mathbf{R}, U, J ; t)} \mathcal{T}_{\mathfrak{v}}}{\partial t}=\frac{\partial \mathbf{E}(\mathbf{R}, t)}{\partial t} \cdot \frac{\widehat{\partial}_{(\mathbf{R}, U, J ; t)} \mathcal{T}_{\mathfrak{v}}}{\partial \mathbf{E}(\mathbf{R}, t)}+\frac{\partial \mathbf{B}(\mathbf{R}, t)}{\partial t} \cdot \frac{\widehat{\partial}_{(\mathbf{R}, U, J ; t)} \mathcal{T}_{\mathfrak{v}}}{\partial \mathbf{B}(\mathbf{R}, t)} \\
& +\frac{\partial \mathbf{B}_{R_{i}}(\mathbf{R}, t)}{\partial t} \cdot \frac{\widehat{\partial}_{(\mathbf{R}, U, J ; t)} \mathcal{T}_{\mathfrak{v}}}{\partial \mathbf{B}_{, R_{i}}(\mathbf{R}, t)}
\end{aligned}
$$

and

$$
\begin{gathered}
\left.\frac{\widehat{\partial} \mathcal{T}_{\boldsymbol{\rho}}}{\partial t}\right|_{\mathbf{R}, U, J, \phi}=\frac{\widehat{\partial}_{(\mathbf{R}, U, J ; t)} \mathcal{T}_{\boldsymbol{\rho}}}{\partial t} \\
\frac{\widehat{\partial}_{(\mathbf{R}, U, J ; t)} \mathcal{T}_{\boldsymbol{\rho}}}{\partial t}=\frac{\partial \mathbf{B}(\mathbf{R}, t)}{\partial t} \cdot \frac{\widehat{\partial}_{(\mathbf{R}, U, J ; t)} \mathcal{T}_{\boldsymbol{\rho}}}{\partial \mathbf{B}(\mathbf{R}, t)}+\frac{\partial \mathbf{B}_{R_{i}}(\mathbf{R}, t)}{\partial t} \cdot \frac{\widehat{\partial}_{(\mathbf{R}, U, J ; t)} \mathcal{T}_{\boldsymbol{\rho}}}{\partial \mathbf{B}_{R_{i}}(\mathbf{R}, t)} .
\end{gathered}
$$

There is no term $(\partial \mathbf{E}(\mathbf{R}, t) / \partial t) \cdot\left(\widehat{\partial}_{(\mathbf{R}, U, J ; t)} \mathcal{T}_{\boldsymbol{\rho}} / \partial \mathbf{E}(\mathbf{R}, t)\right)$ in the last expression because $\boldsymbol{\rho}$ does not depend on $\mathbf{E}(\mathbf{R}, t)$. 


\section{Appendix B. Transformations used in the deriva- tion of $\delta_{\delta \Phi} \mathcal{L}_{\mathrm{K}}(\mathbf{r}, t)$ and $\delta_{\delta \mathrm{A}} \mathcal{L}_{\mathrm{K}}(\mathbf{r}, t)$}

The generally valid relation $g(x) \delta\left(x-x_{0}\right)=g\left(x_{0}\right) \delta\left(x-x_{0}\right)$, for any function $g(x)$, can be used to transform several expressions which appear in $\delta_{\delta \Phi} \mathcal{L}_{\mathrm{K}}(\mathbf{r}, t)$ and $\delta_{\delta \mathbf{A}} \mathcal{L}_{\mathrm{K}}(\mathbf{r}, t)$. Owing to the properties of the delta function, it is possible to write the terms in different, equivalent forms. The following are, therefore, not the only possible expressions.

The following expressions are needed for evaluation of $\delta_{\delta \Phi} \mathcal{L}_{\mathrm{K}}(\mathbf{r}, t)$ :

$$
\begin{aligned}
& \mathcal{B}(\mathbf{R}, U, J ; t) f(\mathbf{R}, U, J ; t) \delta(\mathbf{r}-\mathbf{R}) \frac{\partial \delta \Phi(\mathbf{R}, t)}{\partial \mathbf{R}} \cdot \frac{\widehat{\partial}_{(\mathbf{R}, U, J ; t)}\left\langle\mathcal{T}_{\mathfrak{v}}\right\rangle}{\partial \mathbf{E}(\mathbf{R}, t)} \\
&=\quad \mathcal{B}(\mathbf{R}, U, J ; t) f(\mathbf{R}, U, J ; t) \delta(\mathbf{r}-\mathbf{R}) \frac{\partial \delta \Phi(\mathbf{r}, t)}{\partial \mathbf{r}} \cdot \frac{\widehat{\partial}_{(\mathbf{R}, U, J ; t)}\left\langle\mathcal{T}_{\mathfrak{v}}\right\rangle}{\partial \mathbf{E}(\mathbf{R}, t)} \\
&=\frac{\partial}{\partial \mathbf{r}} \cdot\left[\delta(\mathbf{r}-\mathbf{R}) \delta \Phi(\mathbf{r}, t) \mathcal{B}(\mathbf{R}, U, J ; t) f(\mathbf{R}, U, J ; t) \frac{\widehat{\partial}_{(\mathbf{R}, U, J ; t)}\left\langle\mathcal{T}_{\mathfrak{v}}\right\rangle}{\partial \mathbf{E}(\mathbf{R}, t)}\right] \\
&-\delta \Phi(\mathbf{r}, t) \frac{\partial}{\partial \mathbf{r}} \cdot\left[\delta(\mathbf{r}-\mathbf{R}) \mathcal{B}(\mathbf{R}, U, J ; t) f(\mathbf{R}, U, J ; t) \frac{\widehat{\partial}_{(\mathbf{R}, U, J ; t)}\left\langle\mathcal{T}_{\mathfrak{v}}\right\rangle}{\partial \mathbf{E}(\mathbf{R}, t)}\right],(B)
\end{aligned}
$$

and

$$
\begin{aligned}
& \mathcal{B}(\mathbf{R}, U, J ; t) f(\mathbf{R}, U, J ; t)\left\langle\delta(\mathbf{r}-\mathfrak{x}) \frac{\partial \delta \Phi(\mathfrak{x}, t)}{\partial \mathfrak{x}} \cdot \frac{\widehat{\partial}_{(\mathfrak{x}, t)} \mathcal{T}_{\mathfrak{v}}}{\partial \mathbf{E}(\mathfrak{x}, t)}\right\rangle \\
&= \mathcal{B}(\mathbf{R}, U, J ; t) f(\mathbf{R}, U, J ; t)\left\langle\delta(\mathbf{r}-\mathfrak{x}) \frac{\partial \delta \Phi(\mathbf{r}, t)}{\partial \mathbf{r}} \cdot \frac{\widehat{\partial}_{(\mathfrak{x}, t)} \mathcal{T}_{\mathfrak{v}}}{\partial \mathbf{E}(\mathfrak{x}, t)}\right\rangle \\
&= \frac{\partial}{\partial \mathbf{r}} \cdot\left[\delta \Phi(\mathbf{r}, t) \mathcal{B}(\mathbf{R}, U, J ; t) f(\mathbf{R}, U, J ; t)\left\langle\delta(\mathbf{r}-\mathfrak{x}) \frac{\widehat{\partial}_{(\mathfrak{x}, t)} \mathcal{T}_{\mathfrak{v}}}{\partial \mathbf{E}(\mathfrak{x}, t)}\right\rangle\right] \\
&-\delta \Phi(\mathbf{r}, t) \frac{\partial}{\partial \mathbf{r}} \cdot\left[\mathcal{B}(\mathbf{R}, U, J ; t) f(\mathbf{R}, U, J ; t)\left\langle\delta(\mathbf{r}-\mathfrak{x}) \frac{\widehat{\partial}_{(\mathfrak{x}, t)} \mathcal{T}_{\mathfrak{v}}}{\partial \mathbf{E}(\mathfrak{x}, t)}\right\rangle\right]
\end{aligned}
$$

As in (85), some terms in (87) can be transformed by setting $\delta(\mathbf{r}-\mathbf{R}) g(\mathfrak{x})=\delta(\mathbf{r}-\mathfrak{x}) g(\mathfrak{x})+[\delta(\mathbf{r}-\mathbf{R})-\delta(\mathbf{r}-\mathfrak{x})] g(\mathfrak{x})$. The following terms then appear in $\delta_{\delta \mathbf{A}} \mathcal{L}_{\mathrm{K}}(\mathbf{r}, t)$ :

$$
\begin{aligned}
\left.\delta(\mathbf{r}-\mathbf{R}) \mathcal{B} f \frac{1}{c} \frac{\partial \delta \mathbf{A}(\mathbf{R}, t)}{\partial t}\right|_{\mathbf{R}} \cdot \frac{\widehat{\partial}_{(\mathbf{R}, U, J ; t)}\left\langle\mathcal{T}_{\mathfrak{v}}\right\rangle}{\partial \mathbf{E}(\mathbf{R}, t)} \\
=\left.\quad \delta(\mathbf{r}-\mathbf{R}) \mathcal{B} f \frac{1}{c} \frac{\partial \delta \mathbf{A}(\mathbf{r}, t)}{\partial t}\right|_{\mathbf{r}} \cdot \frac{\widehat{\partial}_{(\mathbf{R}, U, J ; t)}\left\langle\mathcal{T}_{\mathfrak{v}}\right\rangle}{\partial \mathbf{E}(\mathbf{R}, t)} \\
=\left.\frac{1}{c} \frac{\partial}{\partial t}\right|_{\mathbf{r}}\left[\delta \mathbf{A}(\mathbf{r}, t) \cdot \mathcal{B} f \delta(\mathbf{r}-\mathbf{R}) \frac{\widehat{\partial}_{(\mathbf{R}, U, J ; t)}\left\langle\mathcal{T}_{\mathfrak{v}}\right\rangle}{\partial \mathbf{E}(\mathbf{R}, t)}\right] \\
-\left.\delta \mathbf{A}(\mathbf{r}, t) \cdot \frac{1}{c} \frac{\partial}{\partial t}\right|_{\mathbf{r}}\left[\mathcal{B} f \delta(\mathbf{r}-\mathbf{R}) \frac{\widehat{\partial}_{(\mathbf{R}, U, J ; t)}\left\langle\mathcal{T}_{\mathfrak{v}}\right\rangle}{\partial \mathbf{E}(\mathbf{R}, t)}\right],
\end{aligned}
$$




$$
\begin{aligned}
& \left.\delta(\mathbf{r}-\mathfrak{x}) \mathcal{B} f \frac{1}{c} \frac{\partial \delta \mathbf{A}(\mathfrak{x}, t)}{\partial t}\right|_{\mathfrak{x}} \cdot \frac{\widehat{\partial}_{(\mathfrak{x}, t)} \mathcal{T}_{\mathfrak{v}}}{\partial \mathbf{E}(\mathfrak{x}, t)} \\
& =\left.\delta(\mathbf{r}-\mathfrak{x}) \mathcal{B} f \frac{1}{c} \frac{\partial \delta \mathbf{A}(\mathbf{r}, t)}{\partial t}\right|_{\mathbf{r}} \cdot \frac{\widehat{\partial}_{(\mathfrak{x}, t)} \mathcal{T}_{\mathfrak{v}}}{\partial \mathbf{E}(\mathfrak{x}, t)} \\
& =\left.\frac{1}{c} \frac{\partial}{\partial t}\right|_{\mathbf{r}}\left[\delta \mathbf{A}(\mathbf{r}, t) \cdot \mathcal{B} f \delta(\mathbf{r}-\mathfrak{x}) \frac{\widehat{\partial}_{(\mathfrak{x}, t)} \mathcal{T}_{\mathfrak{v}}}{\partial \mathbf{E}(\mathfrak{x}, t)}\right] \\
& -\left.\delta \mathbf{A}(\mathbf{r}, t) \cdot \frac{1}{c} \frac{\partial}{\partial t}\right|_{\mathbf{r}}\left[\mathcal{B} f \delta(\mathbf{r}-\mathfrak{x}) \frac{\widehat{\partial}_{(\mathfrak{x}, t)} \mathcal{T}_{\mathfrak{v}}}{\partial \mathbf{E}(\mathfrak{x}, t)}\right], \\
& \delta(\mathbf{r}-\mathbf{R}) \mathcal{B} f\left(\boldsymbol{\nabla}_{\mathbf{R}} \times \delta \mathbf{A}(\mathbf{R}, t)\right) \cdot \frac{\widehat{\partial}_{(\mathbf{R}, U, J ; t)}\langle\mathcal{T}\rangle}{\partial \mathbf{B}(\mathbf{R}, t)} \\
& =\delta(\mathbf{r}-\mathbf{R}) \mathcal{B} f\left(\boldsymbol{\nabla}_{\mathbf{r}} \times \delta \mathbf{A}(\mathbf{r}, t)\right) \cdot \frac{\widehat{\partial}_{(\mathbf{R}, U, J ; t)}\langle\mathcal{T}\rangle}{\partial \mathbf{B}(\mathbf{R}, t)} \\
& =\frac{\partial}{\partial \mathbf{r}} \cdot\left[\delta \mathbf{A}(\mathbf{r}, t) \times \mathcal{B} f \delta(\mathbf{r}-\mathbf{R}) \frac{\widehat{\partial}_{(\mathbf{R}, U, J ; t)}\langle\mathcal{T}\rangle}{\partial \mathbf{B}(\mathbf{R}, t)}\right] \\
& =\delta \mathbf{A}(\mathbf{r}, t) \cdot \nabla_{\mathbf{r}} \times\left[\mathcal{B} f \delta(\mathbf{r}-\mathbf{R}) \frac{\widehat{\partial}_{(\mathbf{R}, U, J ; t)}\langle\mathcal{T}\rangle}{\partial \mathbf{B}(\mathbf{R}, t)}\right], \\
& \delta(\mathbf{r}-\mathfrak{x}) \mathcal{B} f\left(\nabla_{\mathfrak{x}} \times \delta \mathbf{A}(\mathfrak{x}, t)\right) \cdot \frac{\widehat{\partial}_{(\mathfrak{x}, t)} \mathcal{T}}{\partial \mathbf{B}(\mathfrak{x}, t)} \\
& =\delta(\mathbf{r}-\mathfrak{x}) \mathcal{B} f\left(\nabla_{\mathbf{r}} \times \delta \mathbf{A}(\mathbf{r}, t)\right) \cdot \frac{\widehat{\partial}_{(\mathfrak{x}, t)} \mathcal{T}}{\partial \mathbf{B}(\mathfrak{x}, t)} \\
& =\frac{\partial}{\partial \mathbf{r}} \cdot\left[\delta \mathbf{A}(\mathbf{r}, t) \times \mathcal{B} f \delta(\mathbf{r}-\mathfrak{x}) \frac{\widehat{\partial}_{(x, t)} \mathcal{T}}{\partial \mathbf{B}(\mathfrak{x}, t)}\right] \\
& =\delta \mathbf{A}(\mathbf{r}, t) \cdot \nabla_{\mathbf{r}} \times\left[\mathcal{B} f \delta(\mathbf{r}-\mathfrak{x}) \frac{\widehat{\partial}_{(\mathfrak{x}, t)} \mathcal{T}}{\partial \mathbf{B}(\mathfrak{x}, t)}\right], \\
& \delta(\mathbf{r}-\mathbf{R}) \mathcal{B} f\left(\frac{\partial}{\partial R_{i}} \nabla_{\mathbf{R}} \times \delta \mathbf{A}(\mathbf{R}, t)\right) \cdot \frac{\widehat{\partial}_{(\mathbf{R}, U, J ; t)}\langle\mathcal{T}\rangle}{\partial \mathbf{B}_{R_{i}}(\mathbf{R}, t)} \\
& =\delta(\mathbf{r}-\mathbf{R}) \mathcal{B} f\left(\frac{\partial}{\partial r_{i}} \boldsymbol{\nabla}_{\mathbf{r}} \times \delta \mathbf{A}(\mathbf{r}, t)\right) \cdot \frac{\widehat{\partial}_{(\mathbf{R}, U, J ; t)}\langle\mathcal{T}\rangle}{\partial \mathbf{B}_{R_{i}}(\mathbf{R}, t)} \\
& =\frac{\partial}{\partial r_{i}}\left[\mathcal{B} f\left(\nabla_{\mathbf{r}} \times \delta \mathbf{A}(\mathbf{r}, t)\right) \cdot \delta(\mathbf{r}-\mathbf{R}) \frac{\widehat{\partial}_{(\mathbf{R}, U, J ; t)}\langle\mathcal{T}\rangle}{\partial \mathbf{B}_{,_{i}}(\mathbf{R}, t)}\right] \\
& -\left(\boldsymbol{\nabla}_{\mathbf{r}} \times \delta \mathbf{A}(\mathbf{r}, t)\right) \cdot \frac{\partial}{\partial r_{i}}\left[\mathcal{B} f \delta(\mathbf{r}-\mathbf{R}) \frac{\widehat{\partial}_{(\mathbf{R}, U, J ; t)}\langle\mathcal{T}\rangle}{\partial \mathbf{B}_{R_{i}}(\mathbf{R}, t)}\right] \\
& =\frac{\partial}{\partial r_{i}}\left[\mathcal{B} f\left(\boldsymbol{\nabla}_{\mathbf{r}} \times \delta \mathbf{A}(\mathbf{r}, t)\right) \cdot \delta(\mathbf{r}-\mathbf{R}) \frac{\widehat{\partial}_{(\mathbf{R}, U, J ; t)}\langle\mathcal{T}\rangle}{\partial \mathbf{B}_{R_{i}}(\mathbf{R}, t)}\right]
\end{aligned}
$$




$$
\begin{aligned}
& -\frac{\partial}{\partial \mathbf{r}} \cdot\left[\delta \mathbf{A}(\mathbf{r}, t) \times \frac{\partial}{\partial r_{i}}\left[\mathcal{B} f \delta(\mathbf{r}-\mathbf{R}) \frac{\widehat{\partial}_{(\mathbf{R}, U, J ; t)}\langle\mathcal{T}\rangle}{\partial \mathbf{B}_{, R_{i}}(\mathbf{R}, t)}\right]\right] \\
& -(\delta \mathbf{A}(\mathbf{r}, t)) \cdot \boldsymbol{\nabla}_{\mathbf{r}} \times \frac{\partial}{\partial r_{i}}\left[\mathcal{B} f \delta(\mathbf{r}-\mathbf{R}) \frac{\widehat{\partial}_{(\mathbf{R}, U, J ; t)}\langle\mathcal{T}\rangle}{\partial \mathbf{B}_{, R_{i}}(\mathbf{R}, t)}\right], \\
& \delta(\mathbf{r}-\mathfrak{x}) \mathcal{B} f\left(\frac{\partial}{\partial \mathrm{x}_{\mathrm{i}}} \nabla_{\mathfrak{x}} \times \delta \mathbf{A}(\mathfrak{x}, t)\right) \cdot \frac{\widehat{\partial}_{(\mathfrak{x}, t)}\langle\mathcal{T}\rangle}{\partial \mathbf{B}_{, \mathrm{x}_{\mathrm{i}}}(\mathfrak{x}, t)} \\
& =\delta(\mathbf{r}-\mathfrak{x}) \mathcal{B} f\left(\frac{\partial}{\partial r_{i}} \boldsymbol{\nabla}_{\mathbf{r}} \times \delta \mathbf{A}(\mathbf{r}, t)\right) \cdot \frac{\widehat{\partial}_{(\mathfrak{x}, t)}\langle\mathcal{T}\rangle}{\partial \mathbf{B}_{\mathrm{x}_{\mathrm{i}}}(\mathfrak{x}, t)} \\
& =\frac{\partial}{\partial r_{i}}\left[\mathcal{B} f\left(\nabla_{\mathbf{r}} \times \delta \mathbf{A}(\mathbf{r}, t)\right) \cdot \delta(\mathbf{r}-\mathfrak{x}) \frac{\widehat{\partial}_{(\mathfrak{x}, t)}\langle\mathcal{T}\rangle}{\partial \mathbf{B}_{\mathrm{x}_{\mathrm{i}}}(\mathfrak{x}, t)}\right] \\
& -\left(\boldsymbol{\nabla}_{\mathbf{r}} \times \delta \mathbf{A}(\mathbf{r}, t)\right) \cdot \frac{\partial}{\partial r_{i}}\left[\mathcal{B} f \delta(\mathbf{r}-\mathfrak{x}) \frac{\widehat{\partial}_{(\mathfrak{x}, t)}\langle\mathcal{T}\rangle}{\partial \mathbf{B}_{,_{\mathrm{i}}}(\mathfrak{x}, t)}\right] \\
& =\frac{\partial}{\partial r_{i}}\left[\mathcal{B} f\left(\boldsymbol{\nabla}_{\mathbf{r}} \times \delta \mathbf{A}(\mathbf{r}, t)\right) \cdot \delta(\mathbf{r}-\mathfrak{x}) \frac{\widehat{\partial}_{(\mathfrak{x}, t)}\langle\mathcal{T}\rangle}{\partial \mathbf{B}_{,_{\mathrm{x}}}(\mathfrak{x}, t)}\right] \\
& -\frac{\partial}{\partial \mathbf{r}} \cdot\left[\delta \mathbf{A}(\mathbf{r}, t) \times \frac{\partial}{\partial r_{i}}\left[\mathcal{B} f \delta(\mathbf{r}-\mathfrak{x}) \frac{\widehat{\partial}_{(\mathfrak{x}, t)}\langle\mathcal{T}\rangle}{\partial \mathbf{B}_{, \mathrm{x}_{\mathrm{i}}}(\mathfrak{x}, t)}\right]\right] \\
& -(\delta \mathbf{A}(\mathbf{r}, t)) \cdot \nabla_{\mathbf{r}} \times \frac{\partial}{\partial r_{i}}\left[\mathcal{B} f \delta(\mathbf{r}-\mathfrak{x}) \frac{\widehat{\partial}_{(\mathfrak{x}, t)}\langle\mathcal{T}\rangle}{\partial \mathbf{B}_{, \mathrm{x}_{\mathrm{i}}}(\mathfrak{x}, t)}\right] .
\end{aligned}
$$

\section{Appendix C. Gauge-invariant shift variations}

The goal here is to describe the effect of shifts in space and time by gaugeinvariant shift variations of the quantities which appear in $\delta \mathcal{L}(\mathbf{r}, t)$. One thus directly obtains the necessarily gauge-invariant expressions entering the local energy, momentum and angular momentum conservation laws. This is done as explained in detail in [14], though the problem of interest here has additional difficulties because in some terms the potentials depend on $(\mathbf{R}, t)$, and in other terms on $(\mathfrak{x}(\mathbf{R}, U, J, \phi ; t), t)$. This requires special treatment.

We consider here Eulerian variations of scalars and vectors, brought about by infinitesimal time shifts, spatial translations and rotations of the whole system to which they belong.

Let the shifted quantities be characterized by an asterisk *. Any point $\mathbf{r}$ of the unshifted system is moved to a corresponding, shifted point $\mathbf{r}^{*}$ given by

$$
\mathbf{r}^{*}=\mathbf{r}+\boldsymbol{\zeta}_{\mathbf{r}}, \quad \boldsymbol{\zeta}_{\mathbf{r}} \equiv \boldsymbol{\epsilon}_{\text {trans. }}+\boldsymbol{\epsilon}_{\mathrm{rot} .} \times \mathbf{r}
$$

where $\boldsymbol{\epsilon}_{\text {trans. }}$ and $\boldsymbol{\epsilon}_{\text {rot. }}$ are constant vectors. The new time in the shifted system relates to the old time in the unshifted system according to

$$
t^{*}=t+\epsilon_{t}
$$


$\epsilon_{t}$ being a constant. In the present context we consider arbitrary points $\mathbf{r}$. In particular, we consider the gyrocentre position $\mathbf{R}$ and the particle position $\mathfrak{x}$. For any scalar quantity, the new value at the shifted position and time is equal to the old value at the unshifted position and time:

$$
\Phi^{*}\left(\mathbf{r}^{*}, t^{*}\right)=\Phi(\mathbf{r}, t) .
$$

The Eulerian variation of a quantity is defined as the difference between the new and old values, both taken at the old, unshifted point and time. For a scalar, one has

$$
\begin{aligned}
\delta_{\text {shift }} \Phi(\mathbf{r}, t) & =\Phi^{*}(\mathbf{r}, t)-\Phi(\mathbf{r}, t) \\
& =-\boldsymbol{\zeta}_{\mathbf{r}} \cdot \frac{\partial \Phi(\mathbf{r}, t)}{\partial \mathbf{r}}-\epsilon_{t} \frac{\partial \Phi(\mathbf{r}, t)}{\partial t} .
\end{aligned}
$$

Any vector $\mathbf{w}(\mathbf{r}, t)$ transforms like the gradient of a scalar. The transformation law is obtained in the following way. The relation

$$
\frac{\partial}{\partial \mathbf{r}} \Phi^{*}\left(\mathbf{r}^{*}, t^{*}\right)=\frac{\partial}{\partial \mathbf{r}} \Phi(\mathbf{r}, t)
$$

is valid because of $(\mathrm{C} 3)$. Then, by identifying the vector $\mathbf{w}(\mathbf{r}, t)$ with the gradient of $\Phi$,

$$
\mathbf{w}(\mathbf{r}, t) \equiv \frac{\partial \Phi(\mathbf{r}, t)}{\partial \mathbf{r}}
$$

one obtains

$$
\mathbf{w}^{*}\left(\mathbf{r}^{*}, t^{*}\right)=\frac{\partial \Phi^{*}\left(\mathbf{r}^{*}, t^{*}\right)}{\partial \mathbf{r}^{*}}=\frac{\partial \mathbf{r}}{\partial \mathbf{r}^{*}} \cdot \frac{\partial \Phi^{*}\left(\mathbf{r}^{*}, t^{*}\right)}{\partial \mathbf{r}}=\frac{\partial \mathbf{r}}{\partial \mathbf{r}^{*}} \cdot \frac{\partial \Phi(\mathbf{r}, t)}{\partial \mathbf{r}}=\frac{\partial \mathbf{r}}{\partial \mathbf{r}^{*}} \cdot \mathbf{w}(\mathbf{r}, t) .
$$

With $\mathbf{r}^{*}$ expressed through $\mathbf{r}$ by inverting (C1), this yields

$$
\mathbf{w}^{*}\left(\mathbf{r}^{*}, t^{*}\right)=\mathbf{w}(\mathbf{r}, t)+\boldsymbol{\epsilon}_{\text {rot. }} \times \mathbf{w}(\mathbf{r}, t) .
$$

With the first-order expansion

$$
\mathbf{w}^{*}\left(\mathbf{r}^{*}, t^{*}\right)=\mathbf{w}^{*}(\mathbf{r}, t)+\boldsymbol{\zeta}_{\mathbf{r}} \cdot \frac{\partial \mathbf{w}(\mathbf{r}, t)}{\partial \mathbf{r}},
$$

the expression for $\delta_{\text {shift }} \mathbf{w}(\mathbf{r}, t)$ is then given by

$$
\delta_{\text {shift }} \mathbf{w}(\mathbf{r}, t)=\mathbf{w}^{*}(\mathbf{r}, t)-\mathbf{w}(\mathbf{r}, t)=-\boldsymbol{\zeta}_{\mathbf{r}} \cdot \frac{\partial \mathbf{w}}{\partial \mathbf{r}}+\boldsymbol{\epsilon}_{\mathrm{rot} .} \times \mathbf{w}-\epsilon_{t} \frac{\partial \mathbf{w}}{\partial t} .
$$

Details concerning these transformations can be found in, for example, Appendix D of [17]. The following properties of the displacements are easily derived:

$$
\nabla_{\mathbf{r}} \cdot \zeta_{\mathbf{r}}=0, \quad \nabla_{\mathbf{r}} \times \zeta_{\mathbf{r}}=2 \epsilon_{\text {rot. }}, \quad\left(\mathbf{w} \cdot \nabla_{\mathbf{r}}\right) \zeta_{\mathbf{r}}=\epsilon_{\text {rot. }} \times \mathbf{w} .
$$


It is important to observe that in scalars and vectors which also depend on the gyroangle $\phi$, and on $U$ and $J$, these three quantities must not be varied, since they are defined relatively to system vectors which are essentially related to the magnetic field. Examples of such vectors are $\boldsymbol{\rho}(\mathbf{R}, U, J, \phi ; t)$ and the particle position $\mathfrak{x}=\mathbf{R}+\boldsymbol{\rho}$. In particular, it is interesting to illustrate the validity of $(\mathrm{C} 10)$ for the variation $\delta_{\text {shift }} \boldsymbol{\rho}$ of $\boldsymbol{\rho}(\mathbf{R}, U, J, \phi ; t)$. This can be seen in the following way. A point

$$
\mathfrak{x}=\mathbf{R}+\boldsymbol{\rho}(\mathbf{R}, U, J, \phi ; t)
$$

is taken by shifts $\boldsymbol{\zeta}_{\mathbf{R}}, \epsilon_{t}$ to a new point

$$
\mathfrak{x}^{*}=\left(\mathbf{R}+\boldsymbol{\zeta}_{\mathbf{R}}\right)+\boldsymbol{\rho}^{*}\left(\mathbf{R}+\boldsymbol{\zeta}_{\mathbf{R}}, U, J, \phi ; t+\epsilon_{t}\right) .
$$

On the other hand, one also has

$$
\begin{aligned}
\mathfrak{x}^{*} & =\mathfrak{x}+\boldsymbol{\zeta}_{\mathfrak{x}} \\
& =\mathbf{R}+\boldsymbol{\rho}(\mathbf{R}, U, J, \phi ; t)+\boldsymbol{\zeta}_{\mathbf{R}}+\boldsymbol{\epsilon}_{\text {rot. }} \times \boldsymbol{\rho}(\mathbf{R}, U, J, \phi ; t)
\end{aligned}
$$

since $\boldsymbol{\zeta}_{\mathfrak{r}}=\boldsymbol{\epsilon}_{\text {rot. }} \times \mathfrak{x}+\boldsymbol{\epsilon}_{\text {trans. }}=\boldsymbol{\zeta}_{\mathrm{R}}+\boldsymbol{\epsilon}_{\text {rot. }} \times \boldsymbol{\rho}$. The equality of the right-hand sides of (C13) and (C14) yields

$$
\boldsymbol{\rho}^{*}\left(\mathbf{R}+\boldsymbol{\zeta}_{\mathbf{R}}, U, J, \phi ; t+\epsilon_{t}\right)=\boldsymbol{\rho}(\mathbf{R}, U, J, \phi ; t)+\boldsymbol{\epsilon}_{\text {rot. }} \times \boldsymbol{\rho}(\mathbf{R}, U, J, \phi ; t),
$$

from which it follows that

$$
\delta_{\text {shift }} \boldsymbol{\rho}=-\boldsymbol{\zeta}_{\mathbf{R}} \cdot \frac{\partial \boldsymbol{\rho}}{\partial \mathbf{R}}+\boldsymbol{\epsilon}_{\mathrm{rot} .} \times \boldsymbol{\rho}-\epsilon_{t} \frac{\partial \boldsymbol{\rho}}{\partial t} .
$$

The origin of this change lies in the fact that the gyromotion at the old point in the shifted system is different from the gyromotion at the old point in the unshifted system.

The shift variations of $\mathbf{A}(\mathfrak{x}, t)$ are

$$
\begin{aligned}
\delta_{\text {shift }} \mathbf{A}(\mathfrak{x}, t)=-\boldsymbol{\zeta}_{\mathfrak{x}} \cdot \frac{\partial \mathbf{A}(\mathfrak{x}, t)}{\partial \mathfrak{x}}+\boldsymbol{\epsilon}_{\text {rot. }} \times \mathbf{A}(\mathfrak{x}, t)-\epsilon_{t} \frac{\partial \mathbf{A}(\mathfrak{x}, t)}{\partial t} \\
=-\frac{\partial}{\partial \mathfrak{x}}\left[\boldsymbol{\zeta}_{\mathfrak{x}} \cdot \mathbf{A}(\mathfrak{x}, t)\right]+\mathbf{A}(\mathfrak{x}, t) \cdot \frac{\partial \boldsymbol{\zeta}_{\mathfrak{x}}}{\partial \mathfrak{x}}+\boldsymbol{\zeta}_{\mathfrak{x}} \times \mathbf{B}(\mathfrak{x}, t) \\
\quad+\mathbf{A}(\mathfrak{x}, t) \times\left(\boldsymbol{\nabla}_{\mathfrak{x}} \times \boldsymbol{\zeta}_{\mathfrak{x}}\right)+\boldsymbol{\epsilon}_{\text {rot. }} \times \mathbf{A}(\mathfrak{x}, t)-\epsilon_{t} \frac{\partial \mathbf{A}(\mathfrak{x}, t)}{\partial t} \\
=\boldsymbol{\zeta}_{\mathfrak{x}} \times \mathbf{B}(\mathfrak{x}, t)+\epsilon_{t} c \mathbf{E}(\mathfrak{x}, t)-\frac{\partial}{\partial \mathfrak{x}}\left[\boldsymbol{\zeta}_{\mathfrak{x}} \cdot \mathbf{A}(\mathfrak{x}, t)-\epsilon_{t} c \Phi(\mathfrak{x}, t)\right],
\end{aligned}
$$

where use was made of (C11). The shift variations of $\Phi(\mathfrak{x}, t)$ are

$$
\begin{aligned}
\delta_{\text {shift }} \Phi(\mathfrak{x}, t) & =-\boldsymbol{\zeta}_{\mathfrak{x}} \cdot \frac{\partial \Phi(\mathfrak{x}, t)}{\partial \mathfrak{x}}-\epsilon_{t} \frac{\partial \Phi(\mathfrak{x}, t)}{\partial t} \\
& =\boldsymbol{\zeta}_{\mathfrak{x}} \cdot \mathbf{E}(\mathfrak{x}, t)+\left.\frac{1}{c} \frac{\partial}{\partial t}\right|_{\mathfrak{x}}\left[\boldsymbol{\zeta}_{\mathfrak{x}} \cdot \mathbf{A}(\mathfrak{x}, t)-\epsilon_{t} c \Phi(\mathfrak{x}, t)\right] .
\end{aligned}
$$


With $\Psi(\mathfrak{x}, t)$ taken as the generating function for an infinitesimal gauge transformation, the gauge variations of $\mathbf{A}(\mathfrak{x}, t)$ and $\Phi(\mathfrak{x}, t)$ are, respectively,

$$
\delta_{\text {gauge }} \mathbf{A}(\mathfrak{x}, t)=\frac{\partial}{\partial \mathfrak{x}} \Psi(\mathfrak{x}, t)
$$

and

$$
\delta_{\text {gauge }} \Phi(\mathfrak{x}, t)=-\frac{1}{c} \frac{\partial}{\partial t} \Psi(\mathfrak{x}, t) .
$$

By choosing the generating function $\Psi(\mathfrak{x}, t)$ for the infinitesimal gauge transformations as

$$
\Psi(\mathfrak{x}, t)=\boldsymbol{\zeta}_{\mathfrak{x}} \cdot \mathbf{A}(\mathfrak{x}, t)-\epsilon_{t} c \Phi(\mathfrak{x}, t),
$$

one then obtains the combined gauge-invariant expressions

$$
\delta_{\text {comb. }} \mathbf{A}(\mathfrak{x}, t)=\delta_{\text {shift }} \mathbf{A}(\mathfrak{x}, t)+\delta_{\text {gauge }} \mathbf{A}(\mathfrak{x}, t)=\boldsymbol{\zeta}_{\mathfrak{x}} \times \mathbf{B}(\mathfrak{x}, t)+\epsilon_{t} c \mathbf{E}(\mathfrak{x}, t)
$$

and

$$
\delta_{\text {comb. }} \Phi(\mathfrak{x}, t)=\delta_{\text {shift }} \Phi(\mathfrak{x}, t)+\delta_{\text {gauge }} \Phi(\mathfrak{x}, t)=\boldsymbol{\zeta}_{\mathfrak{x}} \cdot \mathbf{E}(\mathfrak{x}, t) .
$$

The corresponding expressions with the argument $(\mathbf{r}, t)$ are

$$
\delta_{\text {comb. }} \mathbf{A}(\mathbf{r}, t)=\delta_{\text {shift }} \mathbf{A}(\mathbf{r}, t)+\delta_{\text {gauge }} \mathbf{A}(\mathbf{r}, t)=\boldsymbol{\zeta}_{\mathbf{r}} \times \mathbf{B}(\mathbf{r}, t)+\epsilon_{t} c \mathbf{E}(\mathbf{r}, t)
$$

and

$$
\delta_{\text {comb. }} \Phi(\mathbf{r}, t)=\delta_{\text {shift }} \Phi(\mathbf{r}, t)+\delta_{\text {gauge }} \Phi(\mathbf{r}, t)=\boldsymbol{\zeta}_{\mathbf{r}} \cdot \mathbf{E}(\mathbf{r}, t) .
$$

The shift variation of $S$ is obtained by requiring that the orbits in the shifted system be equal to those in the unshifted system. This means

$$
S^{*}\left(\mathbf{R}+\boldsymbol{\zeta}_{\mathbf{R}}, U ; \alpha_{i} ; J ; t+\epsilon_{t}\right)=S\left(\mathbf{R}, U ; \alpha_{i} ; J ; t\right),
$$

which yields, as expected,

$$
\delta_{\text {shift }} S=-\boldsymbol{\zeta}_{\mathbf{R}} \cdot \frac{\partial S}{\partial \mathbf{R}}-\epsilon_{t} \frac{\partial S}{\partial t} .
$$

After re-introducing $\mathbf{P}_{\mathbf{R}}$ and $P_{U}$ instead of the $\alpha_{i}$, substituting $\partial S / \partial \mathbf{R} \rightarrow \mathbf{P}_{\mathbf{R}}$, $\partial S / \partial t \rightarrow-H_{\mathrm{D}}$ and applying the Dirac constraints $\mathbf{P}_{\mathbf{R}}=(e / c) \hat{\mathbf{A}}$ and $P_{U}=$ (e/c) $\hat{A}_{\mathrm{U}}$, one obtains

$$
\begin{aligned}
\left(\delta_{\text {shift }} S\right)_{\text {constr. }} & =-\boldsymbol{\zeta}_{\mathbf{R}} \cdot\left\langle\frac{\partial \mathfrak{x}}{\partial \mathbf{R}} \cdot \mathfrak{p}\right\rangle-\epsilon_{t}\left\langle\frac{\partial \mathfrak{x}}{\partial t} \cdot \mathfrak{p}\right\rangle+\epsilon_{t}\langle H\rangle \\
& =-\boldsymbol{\zeta}_{\mathbf{R}} \cdot\langle\mathfrak{p}\rangle-\boldsymbol{\zeta}_{\mathbf{R}} \cdot\left\langle\frac{\partial \boldsymbol{\rho}}{\partial \mathbf{R}} \cdot \mathfrak{p}\right\rangle-\epsilon_{t}\left\langle\frac{\partial \boldsymbol{\rho}}{\partial t} \cdot \mathfrak{p}\right\rangle+\epsilon_{t}\langle H\rangle \\
& =-\left\langle\boldsymbol{\zeta}_{\mathfrak{x}} \cdot \mathfrak{p}-\left(\boldsymbol{\epsilon}_{\text {rot. }} \times \boldsymbol{\rho}\right) \cdot \mathfrak{p}\right\rangle-\boldsymbol{\zeta}_{\mathbf{R}} \cdot\left\langle\frac{\partial \boldsymbol{\rho}}{\partial \mathbf{R}} \cdot \mathfrak{p}\right\rangle
\end{aligned}
$$




$$
\begin{aligned}
& -\epsilon_{t}\left\langle\frac{\partial \boldsymbol{\rho}}{\partial t} \cdot \mathfrak{p}\right\rangle+\epsilon_{t}\langle H\rangle \\
= & -\left\langle\boldsymbol{\zeta}_{\mathfrak{x}} \cdot m \mathfrak{v}\right\rangle+\epsilon_{t}\left\langle\frac{m}{2} \mathfrak{v}^{2}\right\rangle \\
& +\left\langle\left(-\epsilon_{t} \frac{\partial \boldsymbol{\rho}}{\partial t}-\boldsymbol{\zeta}_{\mathbf{R}} \cdot \frac{\partial \boldsymbol{\rho}}{\partial \mathbf{R}}+\left(\boldsymbol{\epsilon}_{\text {rot. }} \times \boldsymbol{\rho}\right)\right) \cdot \mathfrak{p}\right\rangle \\
& -\frac{e}{c}\left\langle\boldsymbol{\zeta}_{\mathfrak{x}} \cdot \mathbf{A}(\mathfrak{x}, t)-\epsilon_{t} c \Phi(\mathfrak{x}, t)\right\rangle \\
= & -\left\langle\boldsymbol{\zeta}_{\mathfrak{x}} \cdot m \mathfrak{v}\right\rangle+\epsilon_{t}\left\langle\frac{m}{2} \mathfrak{v}^{2}\right\rangle \\
& +\left\langle\left(\delta_{\text {shift }} \boldsymbol{\rho}\right) \cdot \mathfrak{p}\right\rangle-\frac{e}{c}\langle\Psi(\mathfrak{x}, t)\rangle,
\end{aligned}
$$

where $(\mathrm{C} 16)$ and $(\mathrm{C} 21)$ were used. The change of $S$ owing to a gauge transformation with the generating function $\Psi(\mathfrak{x}, t)$ is given by $(30)$ :

$$
\delta_{\text {gauge }} S=\frac{e}{c}\langle\Psi(\mathfrak{x}, t)\rangle .
$$

Thus, the effect on $S$ of a shift and a simultaneous gauge transformation is

$$
\begin{aligned}
\left(\delta_{\text {comb. }} S\right)_{\text {constr. }}= & \left(\delta_{\text {shift }} S\right)_{\text {constr. }}+\delta_{\text {gauge }} S \\
= & -\left\langle\boldsymbol{\zeta}_{\mathfrak{x}} \cdot m \mathfrak{v}\right\rangle+\epsilon_{t}\left\langle\frac{m}{2} \mathfrak{v}^{2}\right\rangle \\
& +\left\langle\left(\delta_{\text {shift }} \boldsymbol{\rho}\right) \cdot \mathfrak{p}\right\rangle .
\end{aligned}
$$

Owing to the dependence of some terms on $(\mathfrak{x} \equiv \mathbf{R}+\boldsymbol{\rho}, t)$, the quantity entering $\delta \mathcal{L}_{\mathrm{K}}(\mathbf{r}, t)$ is not simply the usual contribution $\delta S$, but $\delta S-\left\langle\left(\delta_{\delta \mathbf{A}} \boldsymbol{\rho}\right) \cdot \mathfrak{p}\right\rangle$, as can be seen by adding the contribution from $\delta_{\delta S}^{\mathrm{EL}} \mathcal{L}_{\mathrm{K}}(\mathbf{r}, t),(83)$, and the $\delta_{\delta \mathbf{A}} \boldsymbol{\rho}$ dependent contribution of $\delta_{\delta \mathbf{A}}^{\mathrm{EL}} \mathcal{L}_{\mathrm{K}}(\mathbf{r}, t)$, given by the last two terms of (89), which include the product $\left(\delta_{\delta \mathbf{A}} \boldsymbol{\rho}\right) \cdot \mathfrak{p}$. For shift variations, $\delta_{\delta \mathbf{A}} \boldsymbol{\rho}$ and $\delta_{\text {shift }} \boldsymbol{\rho}$ are equal since $\boldsymbol{\rho}$ depends on shift-influenced quantities only through $\mathbf{B}$ and its derivatives, and hence on $\mathbf{A}$. This can be explicitly proved at some length. One thus obtains

$$
\begin{aligned}
& \left(\delta_{\text {comb. }} S\right)_{\text {constr. }}-\left\langle\left(\delta_{\delta \mathbf{A}} \boldsymbol{\rho}\right) \cdot \mathfrak{p}\right\rangle \\
& =-\left\langle\boldsymbol{\zeta}_{\mathfrak{x}} \cdot m \mathfrak{v}\right\rangle+\epsilon_{t}\left\langle\frac{m}{2} \mathfrak{v}^{2}\right\rangle+\left\langle\left(\delta_{\text {shift }} \boldsymbol{\rho}-\delta_{\delta \mathbf{A}} \boldsymbol{\rho}\right) \cdot \mathfrak{p}\right\rangle \\
& =-\left\langle\boldsymbol{\zeta}_{\mathfrak{x}} \cdot m \mathfrak{v}\right\rangle+\epsilon_{t}\left\langle\frac{m}{2} \mathfrak{v}^{2}\right\rangle \\
& =-\boldsymbol{\epsilon}_{\text {trans. }} \cdot\langle m \mathfrak{v}\rangle-\boldsymbol{\epsilon}_{\text {rot. }} \cdot\langle\mathfrak{x} \times m \mathfrak{v}\rangle+\epsilon_{t}\left\langle\frac{m}{2} \mathfrak{v}^{2}\right\rangle .
\end{aligned}
$$

\section{Appendix D. Energy conservation from the equa- tions of motion}

In this appendix, energy conservation is derived from the equations of motion

for the gyrocentres. Owing to the complexity of the problem, this is a good 
test of the correctness of the local energy conservation law, (97), derived from the variational principle.

A relation which will be used, valid for functions $\mathcal{F}(\mathbf{R}, U, J, \phi ; t)$, is obtained by multiplying $(72)$ by $\delta(\mathbf{r}-\mathbf{R}) \mathcal{F}(\mathbf{R}, U, J, \phi ; t)$ and integrating in phase space. Since some terms vanish at the boundaries after integration, and with $\partial \delta(\mathbf{r}-$ $\mathbf{R}) / \partial \mathbf{R}=-\partial \delta(\mathbf{r}-\mathbf{R}) / \partial \mathbf{r}$ taken into account, one immediatly obtains the relation

$$
\begin{gathered}
\left.\frac{\partial}{\partial t}\right|_{\mathbf{r}} \int d^{3} R d U d J \mathcal{B} f \delta(\mathbf{r}-\mathbf{R})\langle\mathcal{F}\rangle=\int d^{3} R d U d J \mathcal{B} f \delta(\mathbf{r}-\mathbf{R})\left\langle\frac{\mathfrak{D} \mathcal{F}}{\mathfrak{D} t}\right\rangle \\
-\nabla_{\mathbf{r}} \cdot \int d^{3} R d U d J \mathcal{B} f \delta(\mathbf{r}-\mathbf{R}) \mathbf{V}_{\mathbf{R}}\langle\mathcal{F}\rangle
\end{gathered}
$$

The following equation of motion for gyrocentres was derived in $[1,2]$ :

$$
\hat{\mathbf{E}}-\frac{1}{c} V_{U}\left(\frac{\partial \hat{\mathbf{A}}}{\partial U}-\frac{\partial \hat{A}_{U}}{\partial \mathbf{R}}\right)+\frac{1}{c} \mathbf{V}_{\mathbf{R}} \times \hat{\mathbf{B}}=0 .
$$

The definition of the relevant quantities is given in (5)-(23). Scalar multiplication of (D2) by $\mathbf{V}_{\mathbf{R}}$ and the relation

$$
\mathbf{V}_{\mathbf{R}} \cdot\left(\frac{\partial \hat{\mathbf{A}}}{\partial U}-\frac{\partial \hat{A}_{\mathrm{U}}}{\partial \mathbf{R}}\right)=c\left(\frac{\partial \hat{\Phi}}{\partial U}+\frac{1}{c} \frac{\partial \hat{A}_{\mathrm{U}}}{\partial t}\right)
$$

which is obtained by scalar multiplication of $(5)$ by $\left(\partial \hat{\mathbf{A}} / \partial U-\partial \hat{A}_{\mathrm{U}} / \partial \mathbf{R}\right)$, yields the equation

$$
\mathbf{V}_{\mathbf{R}} \cdot e \hat{\mathbf{E}}-V_{U}\left(e \frac{\partial \hat{\Phi}}{\partial U}+\frac{e}{c} \frac{\partial \hat{A}_{\mathrm{U}}}{\partial t}\right)=0 .
$$

The term $e \hat{\mathbf{E}}$ is

$$
\begin{aligned}
e \hat{\mathbf{E}}= & -\frac{e}{c} \frac{\partial \hat{\mathbf{A}}}{\partial t}-e \frac{\partial \hat{\Phi}}{\partial \mathbf{R}} \\
= & -\left.\frac{\partial}{\partial t}\right|_{\mathbf{R}, U, J}\left\langle\frac{\partial \mathfrak{x}}{\partial \mathbf{R}} \cdot \mathfrak{p}\right\rangle-\left.\frac{\partial}{\partial \mathbf{R}}\right|_{U, J, t}\left\langle\frac{m}{2} \mathfrak{v}^{2}+e \Phi(\mathfrak{x}, t)\right\rangle \\
& +\left.\frac{\partial}{\partial \mathbf{R}}\right|_{\mathbf{R}, U, J}\left\langle\mathfrak{p} \cdot \frac{\partial \mathfrak{x}}{\partial t}\right\rangle \\
= & \left\langle-\left(\frac{\partial}{\partial \mathbf{R}} \frac{\partial \mathfrak{x}}{\partial t}\right) \cdot \mathfrak{p}-\frac{\partial \mathfrak{x}}{\partial \mathbf{R}} \cdot \frac{\partial(m \mathfrak{v})}{\partial t}-\left.\frac{e}{c} \frac{\partial \mathfrak{x}}{\partial \mathbf{R}} \cdot \frac{\partial \mathfrak{x}}{\partial t} \cdot \frac{\partial \mathbf{A}(\mathfrak{x}, t)}{\partial \mathfrak{x}}\right|_{t}\right. \\
& -\left.\frac{e}{c} \frac{\partial \mathfrak{x}}{\partial \mathbf{R}} \cdot \frac{\partial \mathbf{A}(\mathfrak{x}, t)}{\partial t}\right|_{\mathfrak{x}}-\frac{\partial}{\partial \mathbf{R}}\left(\frac{m}{2} \mathfrak{v}^{2}\right)-\left.e \frac{\partial \mathfrak{x}}{\partial \mathbf{R}} \cdot \frac{\partial \Phi(\mathfrak{x}, t)}{\partial \mathfrak{x}}\right|_{t} \\
& +\frac{\partial(m \mathfrak{v})}{\partial \mathbf{R}} \cdot \frac{\partial \mathfrak{x}}{\partial t}+\left(\frac{\partial}{\partial \mathbf{R}} \frac{\partial \mathfrak{x}}{\partial t}\right) \cdot m \mathfrak{v}
\end{aligned}
$$




$$
\begin{aligned}
& \left.+\left.\frac{e}{c} \frac{\partial \mathfrak{x}}{\partial \mathbf{R}} \cdot \frac{\partial \mathbf{A}(\mathfrak{x}, t)}{\partial \mathfrak{x}}\right|_{t} \cdot \frac{\partial \mathfrak{x}}{\partial t}+\frac{e}{c}\left(\frac{\partial}{\partial \mathbf{R}} \frac{\partial \mathfrak{x}}{\partial t}\right) \cdot \mathbf{A}(\mathfrak{x}, t)\right\rangle \\
= & \left\langle\frac{\partial \mathfrak{x}}{\partial \mathbf{R}} \cdot e \mathbf{E}(\mathfrak{x}, t)-\frac{\partial \mathfrak{x}}{\partial \mathbf{R}} \cdot \frac{\partial(m \mathfrak{v})}{\partial t}-\frac{\partial}{\partial \mathbf{R}}\left(\frac{m}{2} \mathfrak{v}^{2}\right)+\frac{\partial(m \mathfrak{v})}{\partial \mathbf{R}} \cdot \frac{\partial \mathfrak{x}}{\partial t}\right. \\
& \left.+\frac{e}{c} \frac{\partial \mathfrak{x}}{\partial \mathbf{R}} \cdot\left(\frac{\partial \mathfrak{x}}{\partial t} \times \mathbf{B}(\mathfrak{x}, t)\right)\right\rangle .
\end{aligned}
$$

By proceeding in a similar way, the term proportional to $V_{U}$ in (D4) yields

$$
\begin{aligned}
-\left(e \frac{\partial \hat{\Phi}}{\partial U}+\frac{e}{c} \frac{\partial \hat{A}_{U}}{\partial t}\right)= & \left\langle\frac{\partial \mathfrak{x}}{\partial U} \cdot e \mathbf{E}(\mathfrak{x}, t)-\frac{\partial \mathfrak{x}}{\partial U} \cdot \frac{\partial(m \mathfrak{v})}{\partial t}-\frac{\partial}{\partial U}\left(\frac{m}{2} \mathfrak{v}^{2}\right)\right. \\
& \left.+\frac{\partial(m \mathfrak{v})}{\partial U} \cdot \frac{\partial \mathfrak{x}}{\partial t}+\frac{e}{c} \frac{\partial \mathfrak{x}}{\partial U} \cdot\left(\frac{\partial \mathfrak{x}}{\partial t} \times \mathbf{B}(\mathfrak{x}, t)\right)\right\rangle .
\end{aligned}
$$

Equation (D4) can then be written as

$$
\begin{aligned}
& \left\langle\left(\mathbf{V}_{\mathbf{R}} \cdot \frac{\partial \mathfrak{x}}{\partial \mathbf{R}}+V_{U} \frac{\partial \mathfrak{x}}{\partial U}\right) \cdot e \mathbf{E}(\mathfrak{x}, t)-\left(\mathbf{V}_{\mathbf{R}} \cdot \frac{\partial \mathfrak{x}}{\partial \mathbf{R}}+V_{U} \frac{\partial \mathfrak{x}}{\partial U}\right) \cdot \frac{\partial(m \mathfrak{v})}{\partial t}\right. \\
& -\left(\mathbf{V}_{\mathbf{R}} \cdot \frac{\partial}{\partial \mathbf{R}}+V_{U} \frac{\partial}{\partial U}\right)\left(\frac{m}{2} \mathfrak{v}^{2}\right)+\left(\mathbf{V}_{\mathbf{R}} \cdot \frac{\partial(m \mathfrak{v})}{\partial \mathbf{R}}+V_{U} \frac{\partial(m \mathfrak{v})}{\partial U}\right) \cdot \frac{\partial \mathfrak{x}}{\partial t} \\
& \left.+\frac{e}{c}\left(\mathbf{V}_{\mathbf{R}} \cdot \frac{\partial \mathfrak{x}}{\partial \mathbf{R}}+V_{U} \frac{\partial \mathfrak{x}}{\partial U}\right) \cdot\left(\frac{\partial \mathfrak{x}}{\partial t} \times \mathbf{B}(\mathfrak{x}, t)\right)\right\rangle=0
\end{aligned}
$$

or else

$$
\begin{aligned}
& \left\langle\left(\frac{\mathfrak{D} \mathfrak{x}}{\mathfrak{D} t}-\frac{\partial \mathfrak{x}}{\partial t}\right) \cdot\left[e \mathbf{E}(\mathfrak{x}, t)-\frac{\partial(m \mathfrak{v})}{\partial t}+\frac{e}{c} \frac{\partial \mathfrak{x}}{\partial t} \times \mathbf{B}(\mathfrak{x}, t)\right]\right. \\
& \left.-\frac{\mathfrak{D}}{\mathfrak{D} t}\left(\frac{m}{2} \mathfrak{v}^{2}\right)+\frac{\partial}{\partial t}\left(\frac{m}{2} \mathfrak{v}^{2}\right)+\left(\frac{\mathfrak{D}(m \mathfrak{v})}{\mathfrak{D} t}-\frac{\partial(m \mathfrak{v})}{\partial t}\right) \cdot \frac{\partial \mathfrak{x}}{\partial t}\right\rangle=0 .
\end{aligned}
$$

With the definition of $\mathbf{G}$ in (A14), this can be expressed as

$$
\begin{aligned}
& \left\langle\frac{\mathfrak{D} \mathfrak{x}}{\mathfrak{D} t} \cdot e \mathbf{E}(\mathfrak{x}, t)-\frac{\mathfrak{D}}{\mathfrak{D} t}\left(\frac{m}{2} \mathfrak{v}^{2}\right)+\frac{\partial}{\partial t}\left(\frac{m}{2} \mathfrak{v}^{2}\right)-\frac{\partial(m \mathfrak{v})}{\partial t} \cdot \frac{\mathfrak{D} \mathfrak{x}}{\mathfrak{D} t}\right. \\
& \left.+\frac{\partial \mathfrak{x}}{\partial t} \cdot\left[\mathbf{G}-\frac{\widehat{\partial}_{(\mathfrak{x}, t)} \mathcal{T}_{\mathfrak{v}}}{\partial \mathfrak{x}}\right]\right\rangle \\
= & \left\langle\frac{\mathfrak{D} \mathfrak{x}}{\mathfrak{D} t} \cdot e \mathbf{E}(\mathfrak{x}, t)-\frac{\mathfrak{D}}{\mathfrak{D} t}\left(\frac{m}{2} \mathfrak{v}^{2}\right)+\left.\frac{\widehat{\partial} \mathcal{T}_{\mathfrak{v}}}{\partial t}\right|_{\mathbf{R}, U, J, \phi}+\left.\frac{\widehat{\partial} \mathcal{T}_{\boldsymbol{\rho}}}{\partial t}\right|_{\mathbf{R}, U, J, \phi}\right. \\
& \left.-\frac{\partial \mathfrak{x}}{\partial t} \cdot \frac{\widehat{\partial}_{(\mathfrak{x}, t)} \mathcal{T}_{\mathfrak{v}}}{\partial \mathfrak{x}}\right\rangle=0,
\end{aligned}
$$

where $\partial \mathfrak{x} / \partial t=\partial \boldsymbol{\rho} / \partial t$ and (A12) and (A15) were used. After multiplication of this equation by $\delta(\mathbf{r}-\mathbf{R}) \mathcal{B} f$, summation over the particle species and 
integration in phase space, one obtains

$$
\begin{aligned}
& \sum_{\text {p. s. }} \int d^{3} R d U d J \mathcal{B} f \delta(\mathbf{r}-\mathbf{R})\left\langle\frac{\mathfrak{D} \mathfrak{x}}{\mathfrak{D} t} \cdot e \mathbf{E}(\mathfrak{x}, t)-\frac{\mathfrak{D}}{\mathfrak{D} t}\left(\frac{m}{2} \mathfrak{v}^{2}\right)\right. \\
& \left.+\left.\frac{\widehat{\partial} \mathcal{T}_{\mathfrak{v}}}{\partial t}\right|_{\mathbf{R}, U, J, \phi}+\left.\frac{\widehat{\partial} \mathcal{T}_{\boldsymbol{\rho}}}{\partial t}\right|_{\mathbf{R}, U, J, \phi}-\frac{\partial \mathfrak{x}}{\partial t} \cdot \frac{\widehat{\partial}_{(\mathfrak{x}, t)} \mathcal{T}_{\mathfrak{v}}}{\partial \mathfrak{x}}\right\rangle=0 .
\end{aligned}
$$

With (65)-(71) and with the substitution

$$
\begin{aligned}
\delta(\mathbf{r}-\mathbf{R}) \mathbf{E}(\mathfrak{x}, t) & =\delta(\mathbf{r}-\mathfrak{x}) \mathbf{E}(\mathfrak{x}, t)+(\delta(\mathbf{r}-\mathbf{R})-\delta(\mathbf{r}-\mathfrak{x})) \mathbf{E}(\mathfrak{x}, t) \\
& =\delta(\mathbf{r}-\mathfrak{x}) \mathbf{E}(\mathbf{r}, t)+(\delta(\mathbf{r}-\mathbf{R})-\delta(\mathbf{r}-\mathfrak{x})) \mathbf{E}(\mathfrak{x}, t)(\mathrm{D} 11)
\end{aligned}
$$

taken into account, the first term in (D10) yields

$$
\begin{aligned}
\sum_{\text {p. s. }} \int & d^{3} R d U d J \mathcal{B} f \delta(\mathbf{r}-\mathbf{R})\left\langle\frac{\mathfrak{D} \mathfrak{x}}{\mathfrak{D} t} \cdot e \mathbf{E}(\mathfrak{x}, t)\right\rangle \\
= & \mathbf{E}(\mathbf{r}, t) \cdot \mathbf{j}_{\text {particle-like }}(\mathbf{r}, t) \\
& +\sum_{\text {p.s. }} \int d^{3} R d U d J \mathcal{B} f\left\langle(\delta(\mathbf{r}-\mathbf{R})-\delta(\mathbf{r}-\mathfrak{x})) e \mathbf{E}(\mathfrak{x}, t) \cdot \frac{\mathfrak{D} \mathfrak{x}}{\mathfrak{D} t}\right\rangle \\
= & \mathbf{E}(\mathbf{r}, t) \cdot\left[\frac{c}{4 \pi} \nabla_{\mathbf{r}} \times \mathbf{B}(\mathbf{r}, t)-\frac{1}{4 \pi} \frac{\partial \mathbf{E}(\mathbf{r}, t)}{\partial t}-\frac{\partial \mathbf{P}(\mathbf{r}, t)}{\partial t}-c \boldsymbol{\nabla}_{\mathbf{r}} \times \mathbf{M}(\mathbf{r}, t)\right] \\
& +\sum_{\text {p.s. }} \int d^{3} R d U d J \mathcal{B} f\left\langle(\delta(\mathbf{r}-\mathbf{R})-\delta(\mathbf{r}-\mathfrak{x})) e \mathbf{E}(\mathfrak{x}, t) \cdot \frac{\mathfrak{D} \mathfrak{x}}{\mathfrak{D} t}\right\rangle \\
= & -\frac{\partial}{\partial t}\left[\frac{1}{8 \pi}\left[\mathbf{E}{ }^{2}(\mathbf{r}, t)+\mathbf{B}{ }^{2}(\mathbf{r}, t)\right]\right]+\mathbf{M}(\mathbf{r}, t) \cdot \frac{\partial \mathbf{B}(\mathbf{r}, t)}{\partial t} \\
& -\nabla_{\mathbf{r}} \cdot\left[\frac{c}{4 \pi} \mathbf{E}(\mathbf{r}, t) \times\left[(\mathbf{B}(\mathbf{r}, t)-4 \pi \mathbf{M}(\mathbf{r}, t)]-\mathbf{E}(\mathbf{r}, t) \cdot \frac{\partial \mathbf{P}(\mathbf{r}, t)}{\partial t}\right.\right. \\
& +\sum_{\text {p.s. }} \int d^{3} R d U d J \mathcal{B} f\left\langle(\delta(\mathbf{r}-\mathbf{R})-\delta(\mathbf{r}-\mathfrak{x})) e \mathbf{E}(\mathfrak{x}, t) \cdot \frac{\mathfrak{D} \mathfrak{x}}{\mathfrak{D} t}\right\rangle .
\end{aligned}
$$

From the term $\mathfrak{D}\left(m \mathfrak{v}^{2} / 2\right) / \mathfrak{D} t$, one obtains

$$
\begin{aligned}
- & \sum_{\text {p. s. }} \int d^{3} R d U d J \mathcal{B} f \delta(\mathbf{r}-\mathbf{R})\left\langle\frac{\mathfrak{D}}{\mathfrak{D} t}\left(\frac{m}{2} \mathfrak{v}^{2}\right)\right\rangle \\
= & -\frac{\partial}{\partial t} \mid \sum_{\mathbf{r}} \text { p. s. } \\
& \int d^{3} R d U d J \mathcal{B} f \delta(\mathbf{r}-\mathbf{R})\left\langle\frac{m}{2} \mathfrak{v}^{2}\right\rangle \\
& -\nabla_{\mathbf{r}} \cdot \sum_{\text {p. s. }} \int d^{3} R d U d J \mathcal{B} f \delta(\mathbf{r}-\mathbf{R}) \mathbf{V}_{\mathbf{R}}\left\langle\frac{m}{2} \mathfrak{v}^{2}\right\rangle,
\end{aligned}
$$

where (D1) was used. 
The $\mathcal{T}_{\mathfrak{v}}$ and $\mathcal{T}_{\boldsymbol{\rho}}$-dependent terms in (D10) yield, with (A21) and (A25) taken into account, the following expression:

$$
\begin{aligned}
& \sum_{\text {p. s. }} \int d^{3} R d U d J \mathcal{B} f \delta(\mathbf{r}-\mathbf{R})\left\langle\left.\frac{\widehat{\partial} \mathcal{T}_{\mathfrak{v}}}{\partial t}\right|_{\mathbf{R}, U, J, \phi}+\left.\frac{\widehat{\partial} \mathcal{T}_{\boldsymbol{\rho}}}{\partial t}\right|_{\mathbf{R}, U, J, \phi}-\frac{\partial \mathfrak{x}}{\partial t} \cdot \frac{\widehat{\partial}_{(\mathfrak{x}, t)} \mathcal{T}_{\mathfrak{v}}}{\partial \mathfrak{x}}\right\rangle \\
& =\sum_{\text {p.s. }} \int d^{3} R d U d J \mathcal{B} f \delta(\mathbf{r}-\mathbf{R})\left\langle\frac{\widehat{\partial}_{(\mathbf{R}, U, J ; t)} \mathcal{T}_{\mathfrak{v}}}{\partial t}+\frac{\widehat{\partial}_{(\mathfrak{x}, t)} \mathcal{T}_{\mathfrak{v}}}{\partial t}+\frac{\widehat{\partial}_{(\mathbf{R}, U, J ; t)} \mathcal{T}_{\boldsymbol{\rho}}}{\partial t}\right\rangle \\
& =\sum_{\text {p. s. }} \int d^{3} R d U d J \mathcal{B} f\left\langle\delta(\mathbf{r}-\mathbf{R}) \frac{\widehat{\partial}_{(\mathbf{R}, U, J ; t)} \mathcal{T}_{\mathfrak{v}}}{\partial t}+\delta(\mathbf{r}-\mathfrak{x}) \frac{\widehat{\partial}_{(\mathfrak{x}, t)} \mathcal{T}_{\mathfrak{v}}}{\partial t}\right. \\
& \left.\delta(\mathbf{r}-\mathbf{R}) \frac{\widehat{\partial}_{(\mathbf{R}, U, J ; t)} \mathcal{T}_{\boldsymbol{\rho}}}{\partial t}+(\delta(\mathbf{r}-\mathbf{R})-\delta(\mathbf{r}-\mathfrak{x})) \frac{\widehat{\partial}_{(\mathfrak{x}, t)} \mathcal{T}_{\mathfrak{v}}}{\partial t}\right\rangle .
\end{aligned}
$$

With (A22)-(A24), (A26) and the definitions of the polarization $\mathbf{P}$ and the magnetization $\mathbf{M}=\mathbf{M}_{1}+\mathbf{M}_{2}, \mathbf{M}_{2}=\partial \mathcal{M}_{i} / \partial r_{i}$ in (62) and (68)-(71), this can be written as

$$
\begin{aligned}
\sum_{\text {p. s. }} \int & d^{3} R d U d J \mathcal{B} f \delta(\mathbf{r}-\mathbf{R})\left\langle\left.\frac{\widehat{\partial} \mathcal{T}_{\mathfrak{v}}}{\partial t}\right|_{\mathbf{R}, U, J, \phi}+\left.\frac{\widehat{\partial} \mathcal{T}_{\boldsymbol{\rho}}}{\partial t}\right|_{\mathbf{R}, U, J, \phi}-\frac{\partial \mathfrak{x}}{\partial t} \cdot \frac{\widehat{\partial}_{(\mathfrak{x}, t)} \mathcal{T}_{\mathfrak{v}}}{\partial \mathfrak{x}}\right\rangle \\
= & -\frac{\partial \mathbf{E}(\mathbf{r}, t)}{\partial t} \cdot \mathbf{P}(\mathbf{r}, t)-\frac{\partial \mathbf{B}(\mathbf{r}, t)}{\partial t} \cdot \mathbf{M}_{1}(\mathbf{r}, t)+\left(\frac{\partial}{\partial r_{i}} \frac{\partial \mathbf{B}(\mathbf{r}, t)}{\partial t}\right) \cdot \mathcal{M}_{i}(\mathbf{r}, t) \\
& +\sum_{\text {p. s. }} \int d^{3} R d U d J \mathcal{B} f\left\langle(\delta(\mathbf{r}-\mathbf{R})-\delta(\mathbf{r}-\mathfrak{x})) \frac{\widehat{\partial}_{(\mathfrak{x}, t)} \mathcal{T}_{\mathfrak{v}}}{\partial t}\right\rangle
\end{aligned}
$$

Here, summation with respect to $i=1,2,3$ is implied. By inserting (D12), (D13) and (D15) in (D10), one obtains the local energy conservation law given by (97). We have thus proved that both the variational principle with Noether's procedure and judicious manipulation of the equations of motion of the gyrocentres lead to the same expression for the local energy conservation law. Noether's method is, however, the more elegant way to obtain conservation laws.

\section{Appendix E. Transforming some expressions in the conservation laws}

In the expressions for the energy, momentum and angular momentum conservation laws, (97), (104) and (112), there are terms containing the difference $(\delta(\mathbf{r}-\mathbf{R})-\delta(\mathbf{r}-\mathfrak{x}))$. These delta functions are to be considered as functions of $\mathbf{r}$ taken at the points $\mathbf{R}$ and $\mathfrak{x}$, respectively. We look for a vector field $\mathbf{d}(\mathbf{r} ; \mathbf{R}, \mathfrak{x})$ satisfying the relation

$$
\boldsymbol{\nabla}_{\mathbf{r}} \cdot \mathbf{d}(\mathbf{r} ; \mathbf{R}, \mathfrak{x})=\delta(\mathbf{r}-\mathbf{R})-\delta(\mathbf{r}-\mathfrak{x})
$$


This equation is formally the same as that for an electrostatic field $4 \pi|e| \mathbf{d}(\mathbf{r} ; \mathbf{R}, \mathfrak{x})$ produced at $\mathbf{r}$ by a positive point charge $|e|$ located at $\mathbf{R}$ and a negative point charge located at $\mathfrak{x}$. The solution for $\mathbf{d}(\mathbf{r} ; \mathbf{R}, \mathfrak{x})$ is the well-known expression

$$
\begin{aligned}
\mathbf{d}(\mathbf{r} ; \mathbf{R}, \mathfrak{x}) & =\frac{1}{4 \pi} \int\left(\delta\left(\mathbf{r}^{\prime}-\mathbf{R}\right)-\delta\left(\mathbf{r}^{\prime}-\mathfrak{x}\right)\right) \frac{\left(\mathbf{r}-\mathbf{r}^{\prime}\right)}{\left|\left(\mathbf{r}-\mathbf{r}^{\prime}\right)\right|^{3}} d^{3} r^{\prime} \\
& =-\frac{1}{4 \pi} \nabla_{\mathbf{r}} \int\left(\delta\left(\mathbf{r}^{\prime}-\mathbf{R}\right)-\delta\left(\mathbf{r}^{\prime}-\mathfrak{x}\right)\right) \frac{1}{\left|\left(\mathbf{r}-\mathbf{r}^{\prime}\right)\right|} d^{3} r^{\prime}
\end{aligned}
$$

Finally, we derive a relation concerning the variation of $\mathcal{T}_{\mathfrak{v}},(\mathrm{A} 10)$, due to variations of $\mathbf{E}(\mathfrak{x}, t), \mathbf{B}(\mathfrak{x}, t)$ and $\mathbf{B}_{\mathrm{x}_{\mathrm{i}}}(\mathfrak{x}, t)$ brought about by infinitesimal rotations $\boldsymbol{\epsilon}_{\text {rot. }} \times \mathfrak{x}$. The results are needed for obtaining a convenient form of the angular momentum conservation law. The expression in question is the following:

$$
\widehat{\delta}_{(\mathfrak{x}, t)} \mathcal{T}_{\mathfrak{v}} \equiv \delta \mathbf{E}(\mathfrak{x}, t) \cdot \frac{\widehat{\partial}_{(\mathfrak{x}, t)} \mathcal{T}_{\mathfrak{v}}}{\partial \mathbf{E}(\mathfrak{x}, t)}+\delta \mathbf{B}(\mathfrak{x}, t) \cdot \frac{\widehat{\partial}_{(\mathfrak{x}, t)} \mathcal{T}_{\mathfrak{v}}}{\partial \mathbf{B}(\mathfrak{x}, t)}+\delta \mathbf{B}_{\mathrm{x}_{\mathrm{i}}}(\mathfrak{x}, t) \cdot \frac{\widehat{\partial}_{(\mathfrak{x}, t)} \mathcal{T}_{\mathfrak{v}}}{\partial \mathbf{B}_{\mathrm{x}_{\mathrm{i}}}(\mathfrak{x}, t)}
$$

with

$$
\begin{aligned}
\widehat{\partial}_{(\mathfrak{x}, t)} \mathcal{T}_{\mathfrak{v}} & =\frac{m}{2} \partial_{(\mathfrak{x}, t)} \mathfrak{v}^{2}-m\left(\partial_{(\mathfrak{x}, t)} \mathfrak{v}\right) \cdot\left[\mathbf{V}_{\mathbf{R}}+\frac{\mathfrak{D} \boldsymbol{\rho}}{\mathfrak{D} t}\right] \\
& =m\left(\partial_{(\mathfrak{x}, t)} \mathfrak{v}\right) \cdot\left[\mathfrak{v}-\frac{\mathfrak{D} \mathfrak{x}}{\mathfrak{D} t}\right],
\end{aligned}
$$

where $\mathfrak{D} \mathfrak{x} / \mathfrak{D} t=\mathbf{V}_{\mathbf{R}}+\mathfrak{D} \boldsymbol{\rho} / \mathfrak{D} t$ was used. Therefore, one has

$$
\begin{aligned}
\widehat{\delta}_{(\mathfrak{x}, t)} \mathcal{T}_{\mathfrak{v}}= & {\left[\delta \mathbf{E}(\mathfrak{x}, t) \cdot \frac{\widehat{\partial}_{(\mathfrak{x}, t)} \mathfrak{v}}{\partial \mathbf{E}(\mathfrak{x}, t)}+\delta \mathbf{B}(\mathfrak{x}, t) \cdot \frac{\widehat{\partial}_{(\mathfrak{x}, t)} \mathfrak{v}}{\partial \mathbf{B}(\mathfrak{x}, t)}\right.} \\
& \left.+\delta \mathbf{B}_{\mathrm{x}_{\mathbf{i}}}(\mathfrak{x}, t) \cdot \frac{\widehat{\partial}_{(\mathfrak{x}, t)} \mathfrak{v}}{\partial \mathbf{B}_{\mathrm{x}_{\mathbf{i}}}(\mathfrak{x}, t)}\right] \cdot m\left[\mathfrak{v}-\frac{\mathfrak{D} \mathfrak{x}}{\mathfrak{D} t}\right]
\end{aligned}
$$

which can also be written as

$$
\widehat{\delta}_{(x, t)} \mathcal{T}_{\mathfrak{v}}=\left(\delta_{\delta \mathbf{E}(x, t), \ldots} \mathfrak{v}\right) \cdot m\left[\mathfrak{v}-\frac{\mathfrak{D} \mathfrak{x}}{\mathfrak{D} t}\right] .
$$

The vector $\mathfrak{v}$ is given by (21) and (22), and one obtains

$$
\begin{aligned}
\left(\delta_{\delta \mathbf{E}(\mathfrak{x}, t), \ldots} \mathfrak{v}\right)= & u_{\|} \delta \mathbf{b}(\mathfrak{x}, t)+u_{\perp}\left[-\sin \theta \delta \mathbf{e}_{1}(\mathfrak{x}, t)-\cos \theta \delta \mathbf{e}_{2}(\mathfrak{x}, t)\right] \\
& +\delta\left[c \frac{\mathbf{E}(\mathfrak{x}, t) \times \mathbf{B}(\mathfrak{x}, t)}{\mathbf{B}(\mathfrak{x}, t)^{2}}\right] \\
= & u_{\|}\left[-\left(\boldsymbol{\epsilon}_{\text {rot. }} \times \mathfrak{x}\right) \cdot \frac{\partial \mathbf{b}(\mathfrak{x}, t)}{\partial \mathfrak{x}}+\boldsymbol{\epsilon}_{\text {rot. }} \times \mathbf{b}\right]+\ldots \\
= & -\left(\boldsymbol{\epsilon}_{\text {rot. }} \times \mathfrak{x}\right) \cdot \frac{\partial_{(\mathfrak{x}, t)} \mathfrak{v}(\mathfrak{x}, t)}{\partial \mathfrak{x}}+\boldsymbol{\epsilon}_{\text {rot. }} \times \mathfrak{v} .
\end{aligned}
$$

Together with (E6) this yields

$$
\widehat{\delta}_{(\mathfrak{x}, t)} \mathcal{T}_{\mathfrak{v}}=-\boldsymbol{\epsilon}_{\text {rot. }} \cdot\left[\mathfrak{x} \times \frac{\widehat{\partial}_{(\mathfrak{x}, t)} \mathcal{T}_{\mathfrak{v}}}{\partial \mathfrak{x}}+m \mathfrak{v} \times \frac{\mathfrak{D} \mathfrak{x}}{\mathfrak{D} t}\right] .
$$




\section{References}

[1] Pfirsch, D. and Correa-Restrepo, D., New method of deriving local energy and momentum-conserving Maxwell-collisionless drift-kinetic and gyrokinetic theories I. Report IPP 5/93, Max-Planck-Institut fuer Plasmaphysik, Garching. (2001).

[2] Pfirsch, D. and Correa-Restrepo, D., New method of deriving local energy and momentum-conserving Maxwell-collisionless drift-kinetic and gyrokinetic theories: basic theory. Accepted for publication, J. of Plasma Phys., 70, part 6, 2004.

[3] Littlejohn, R. G., A guiding center Hamiltonian: a new approach. J. Math. Phys. 20, 2445-2458 (1979).

[4] Littlejohn, R. G., Hamiltonian formulation of guiding center motion. Phys. Fluids 24, 1730-1749 (1981).

[5] Littlejohn, R. G., Variational principles of guiding centre motion. J. Plasma Phys. 29, 111-125 (1983).

[6] Weyssow, W. and Balescu, R., Hamiltonian theory of guiding centre motion revisited. J. Plasma Phys. 35, 449-471 (1986).

[7] Brizard, A., Nonlinear gyrokinetic Maxwell-Vlasov equations using magnetic co-ordinates. J. Plasma Phys. 41, 541-559 (1989).

[8] Sugama, H., Gyrokinetic field theory. Phys. Plasmas 7, 466-480 (2000).

[9] Brizard, A., Variational principle for nonlinear gyrokinetic MaxwellVlasov equations. Phys. Plasmas 7, 4816-4822 (2000).

[10] Pfirsch, D., New variational formulation of Maxwell-Vlasov and guiding center theories. Local charge and energy conservation laws. Z. Naturforsch. 39a, 1-8 (1984).

[11] Pfirsch, D. and Morrison, P. J., Local conservation laws for the MaxwellVlasov and collisionless kinetic guiding-center theories. Phys. Rev. A 32, 1714-1721 (1985).

[12] Larsson, J., Hamiltonian and non-Hamiltonian perturbation theory for nearly periodic motion. J. Math. Phys. 27, 495-501 (1986).

[13] Kruskal, M., Asymptotic theory of Hamiltonian and other Systems with all Solutions nearly periodic. J. Math. Phys. 3, 806-828 (1962).

[14] Correa-Restrepo, D. and Pfirsch, D., Noether formalism with gaugeinvariant variations. J. Plasma Phys. , 70, 199-213, (2004). 
[15] Dirac, P. A. M., Lectures on Quantum Mechanics (Belfer Graduate School of Science, Yeshiva University, New York, 1964), Lecture No.1.

[16] Sundermeyer, K., Constrained Dynamics (Springer Verlag, Berlin, 1982), Chapters I and II.

[17] Pfirsch, D. and Correa-Restrepo, D., Collisional drift fluid equations and implications for drift waves. Plasma Phys. Control. Fusion 38, 71-101 (1996). 